oth $y$ :

w.

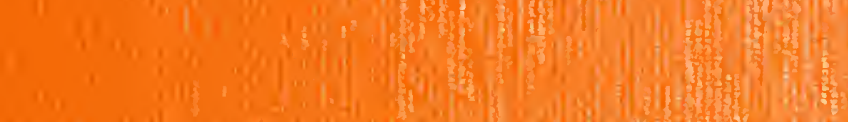

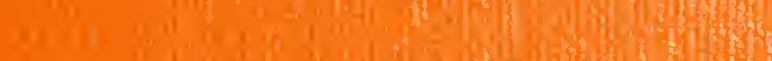

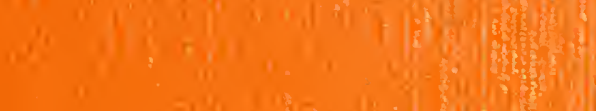

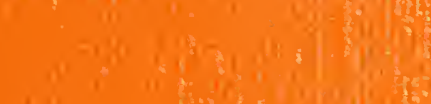
$\left.1^{0}+1+2\right)$

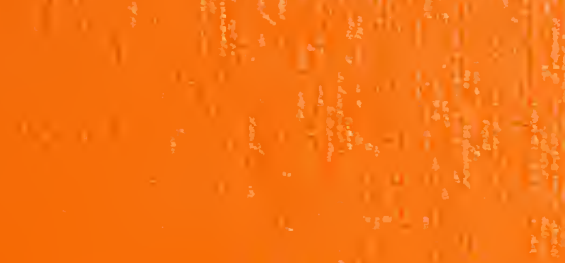

(1)

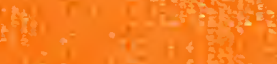

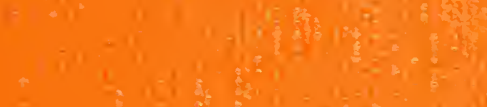

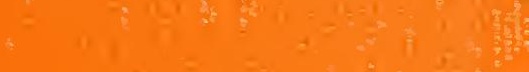

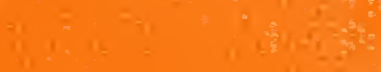

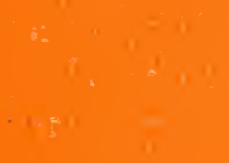

$\because \frac{21}{2}=$

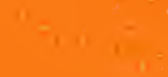

$=t$

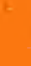

$n$

1)

$\sqrt{2}$

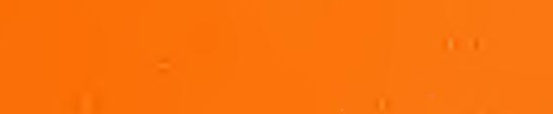

18

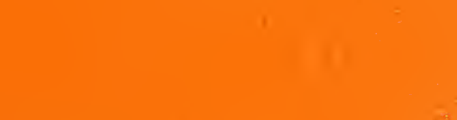

$\left(\frac{1}{2}\right.$

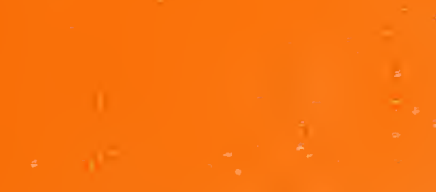

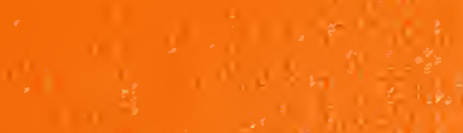

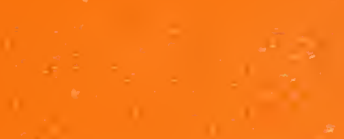

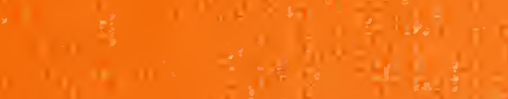

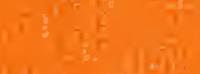

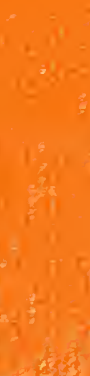

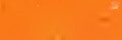
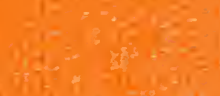

6." in

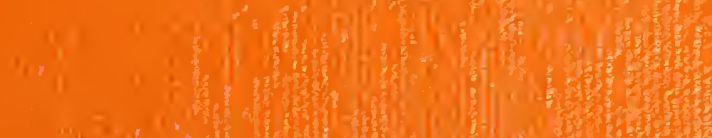

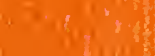

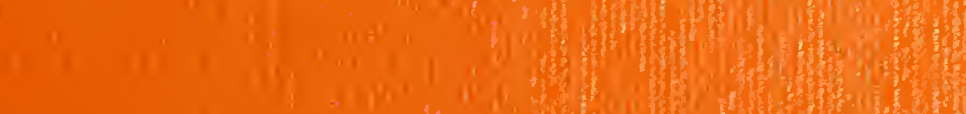

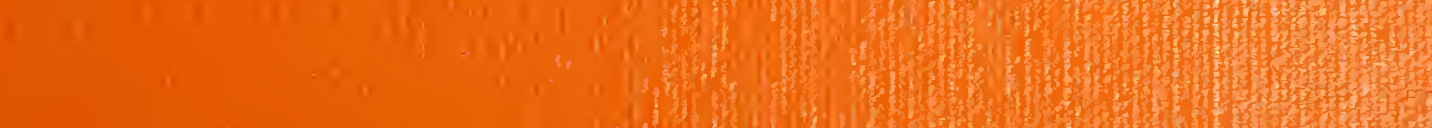





\section{NBSIR 74-577-1 \\ Remittance Processing System, Volume I (Refer to Volume II)}

Philip D. Shupe, Jr.

Paul Meissner

James R. Park

Computer Systems Engineering Division

Institute for Computer Sciences and Technology

National Bureau of Standards

Washington, D. C. 20234

September 1974

Interagency Report

Prepared for

Internal Revenue Service

Systems Development Division

Washington, D. C. 20224 

NBSIR 74-577-1

\section{REMITTANCE PROCESSING SYSTEM, VOLUME I (Refer to Volume II)}

Philip D. Shupe, Jr.

Paul Meissner

James R. Park

Computer Systems Engineering Division

Institute for Computer Sciences and Technology

National Bureau of Standards

Washington, D. C. 20234

September 1974

Interagency Report

Prepared for

Internal Revenue Service

Systems Development Division

Washington, D. C. 20224

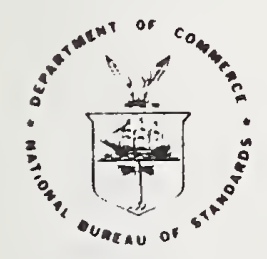

U. S. DEPARTMENT OF COMMERCE, Froderick B. Dent, Secretary NATIONAL BUREAU OF STANDARDS, Richard W. Roberts, Director 


\section{Remittance Processing System \\ Volume I \\ (Reference Volume II)}

TABLE 1

Section I

Section II

Section III

Section IV

Section V

Section VI

Section VII

Section VIII
Glossary of Terms and Abbreviations

The Prototype Remittance Processing System

The Remittance Processing System Controller 31

The RPD Traffic Control Unit

Modification of the Mode1 S/102/S103

Electronic Proof Encoder

Modification of the Mode1 37 Teletype for the RPD

The RPD Control Panel

The RPD Operator's Console

The RPD Document Labeling Device
157

$\frac{\text { Page }}{1}$

\section{6}


Glossary of Terms and Abbreviations

\begin{tabular}{|c|c|}
\hline ACK & Positive acknowledgement \\
\hline $\mathrm{ADCN}$ & Add counter \\
\hline Adv $\emptyset$ & Advance phase \\
\hline AMPR & Amount print \\
\hline ATEN & Audit trail enable \\
\hline $\mathrm{ATF}$ & Audit trail flipflop \\
\hline ATPR & Audit trail print \\
\hline BfRdy & Buffer ready \\
\hline BFIN & Burroughs finished \\
\hline BFINH & Buffer inhibit \\
\hline BRK & Brake \\
\hline $\mathrm{BRPR}$ & Burroughs print \\
\hline $\mathrm{BSY}, \mathrm{BZY}$ & Busy \\
\hline Bt $\emptyset$ & Lowest order character bit \\
\hline Bt 7 & Lateral parity bit \\
\hline $\mathrm{BZ}$ & RPD busy \\
\hline $1 \mathrm{Bf}$ & First buffer ready \\
\hline $2 \mathrm{Bf}$ & Second buffer ready \\
\hline $\mathrm{CC}$ & Communication coupler \\
\hline $\mathrm{CCF}$ & Common character flipflop \\
\hline $\mathrm{CF}$ & Printer command flag \\
\hline $\mathrm{Ch} \mathrm{Cl}$ & Character clock \\
\hline ChP, ChP1 & Character pulse \\
\hline & Clock \\
\hline CL 1 & Control 1ine 1 \\
\hline CL 2 & Control line 2 \\
\hline Clr $1 B f$ & Clear first buffer \\
\hline Clr 2Bf & Clear second buffer \\
\hline CLR BR & Clear Burroughs \\
\hline CLRM & Clear memory \\
\hline CLRM C1 & Clear memory clock \\
\hline CTS & Clear to send \\
\hline DBZ & Detected busy \\
\hline $\mathrm{DcCl}$ & Document clock \\
\hline DCDt & Document data \\
\hline DCEN & Document enable \\
\hline DCFIN & Document finished \\
\hline
\end{tabular}




\begin{tabular}{|c|c|}
\hline DCh & Document character \\
\hline DCLMP & Document lamp \\
\hline DcOK & Document $\mathrm{OK}$ \\
\hline DCPOS & Document positioned \\
\hline DcSp & Document space \\
\hline DCU & Display controller unit \\
\hline DDES & Direct data entry system \\
\hline DEDS & Document edge signal \\
\hline DEL & Delete \\
\hline DERR & Document error \\
\hline DLN & Document line number \\
\hline DLS & Delay symbol \\
\hline DNAK & Detected NAK \\
\hline DOCEDGE & Document edge \\
\hline $\mathrm{DPT}$ & Data print \\
\hline $\mathrm{DPT}$ & Document print \\
\hline DStp & Document stop \\
\hline DTFF & Data transfer flipflop \\
\hline DTR & Data terminal ready \\
\hline EIN & Employer's identification number \\
\hline EOES & End of endorse signal \\
\hline ETX & End of text \\
\hline ETXD & ETX detected \\
\hline FATF & Filtered audit trail flipflop \\
\hline FFF & Forced form feed \\
\hline Fin $\mathrm{Xmt}$ & Finished transmitting \\
\hline FIN & Message finished \\
\hline FWD & Forward \\
\hline GAT & Gated audit trail \\
\hline GDE & Gated document enable \\
\hline GLST & Gated label start \\
\hline GSD & Gated serial data \\
\hline HLTF & Halt forward \\
\hline HLTR & Halt reverse \\
\hline IAT & Inhibit printing of audit trail \\
\hline ICR & Initial condition reset \\
\hline KST & Keyboard station terminal \\
\hline KV & Key verifying \\
\hline
\end{tabular}




$\begin{array}{ll}\text { LABFN } & \text { Label finished } \\ \text { LABST } & \text { Label start } \\ \text { LDAT } & \text { Load audit trail } \\ \text { Ld Bfr } & \text { Load buffer } \\ \text { Ld Bfr } 1 & \text { Load buffer 1 } \\ \text { LDBR } & \text { Load Burroughs } \\ \text { LDCC } & \text { Load command character } \\ \text { Ld Dt } & \text { Load data } \\ \text { Ld Mcr, LDMCR } & \text { Load MICR } \\ \text { LdTTy } & \text { Load Teletype } \\ \text { LGT } & \text { Longitudinal } \\ \text { LGPer } & \text { Longitudinal parity error } \\ \text { LP } & \text { Longitudinal parity } \\ \text { LPMCR } & \text { Load on print MICR } \\ \text { LPStr } & \text { Longitudinal parity strobe } \\ \text { LTPer } & \text { Lateral parity error } \\ \text { LWR } & \text { Lower }\end{array}$

$\begin{array}{ll}\text { Mch } & \text { MICR character } \\ \text { McrC1 } & \text { MICR clock } \\ \text { McrEn } & \text { MICR amount enable } \\ \text { McrOK, MCROK } & \text { MICR OK } \\ \text { MCRPR, McrPr } & \text { MICR print } \\ \text { McSTP } & \text { MICR stop } \\ \text { MRSET, MSTRS } & \text { Master reset }\end{array}$

NAK Negative acknowledgement

NATPR New audit trail print

NFAT New finished audit trail

NoAd No address

NO AT MSG No audit trail message

NoD No more data

$\begin{array}{ll}\text { OE } & \begin{array}{l}\text { Original entry } \\ \text { OK to print }\end{array} \\ \text { PAPOT, PAP } & \begin{array}{l}\text { Paper out } \\ \text { Parallel entry }\end{array} \\ \text { PE } & \begin{array}{l}\text { Print command } \\ \text { PrCND }\end{array} \\ \text { PSC } & \text { Print cycle } \\ & \text { Philadelphia Service Center } \\ \text { RAAC } & \text { Reset all address counters } \\ \text { RMAC } & \text { Reset memory address counter } \\ \text { RMH } & \text { Received message handler }\end{array}$




\begin{tabular}{|c|c|}
\hline ROM & Read only memory \\
\hline RPBR & Repeat Burroughs \\
\hline RPD & Remittance processing device \\
\hline RPS & Remittance processing system \\
\hline RPSC & Remittance processing system controller \\
\hline RptDc & Repeat document \\
\hline RptMcr & Repeat MICR \\
\hline RPTPL & Repeat pulse \\
\hline RptTTy & Repeat Teletype \\
\hline RTS & Request to send signal \\
\hline RZE & Raise \\
\hline $\mathrm{R} \emptyset \mathrm{C}$ & Reset phase counter \\
\hline SBZ & Selected station busy \\
\hline SCTS, STCS & Station clear to send \\
\hline SelBf & Select buffer \\
\hline SelRom & Select ROM address counter \\
\hline Se1Sta & Select station \\
\hline SEO & Second entry operator \\
\hline SHER & Shear \\
\hline Sld & Serial load \\
\hline SldC1 & Serial load clock \\
\hline SMD & Shoe motor drive \\
\hline SMR & Shoe motor \\
\hline $\mathrm{SOH}$ & ASCII start of header code \\
\hline $\mathrm{SPCH}$ & Space character \\
\hline SrDt & Serial data \\
\hline SSN & Social security number \\
\hline Sta Rdy & Station ready \\
\hline STEP & Step \\
\hline STEP B & Step backward \\
\hline STEP F & Step forward \\
\hline STMP & Stamp \\
\hline STMPD & Stamp drive \\
\hline STPCL & Step clock \\
\hline sTX & ASCII start of text code \\
\hline SXCL & Station transmit clock \\
\hline SYN & ASCII synchronizing code \\
\hline SYNFF & Synchronize flipflop \\
\hline $\mathrm{T} / \mathrm{C}$ & Transaction code \\
\hline TETX & Teletype end of text \\
\hline TIN & Taxpayer's identification number \\
\hline TMH & Transmitted message handler \\
\hline $\mathrm{T} / \mathrm{P}$ & Taxpayer \\
\hline TTY & Teletype printer \\
\hline TTyEn & Teletype enable \\
\hline TTYOK & Teletype oK \\
\hline
\end{tabular}


VAC

Vacuum

VCDT

Vacuum detect

$\mathrm{XChCl}$

Transmitted character clock

$\mathrm{XmBf}$ Transmit from buffer

XmtBf

Transmit buffer

XmtC1

Transmit clock

XmtSta

Transmit station

NOTE: In several instances precise identification of a product(s) has been made because the product is a part of an assembly or device and is a part whose physical dimensions or technical characteristics must, in the even of injury or failure, be duplicated exactly in a successful replacement part. In no case does such identification imply recommendation or endorsement by the National Bureau of Standards, nor does it imply that the material or equipment identified is necessarily the best available for the purpose. 
The Prototype Remittance Processing System

\section{Table of Contents}

1.0 Introduction

2.0 Environment

3.0 System Components

3.1 General

3.2 The RPSC

3.3 The RPD

4.0 System Functions

4.1 Message Reception

4.2 Message Transmission

5.0 References

\section{List of Figures}

1.1 Photograph of RPD

1.2 Photograph of RPS Controller

3.1 Remittance Processing System Block Diagram

4.1 Format of Message from Computer to RPD

4.2 Format of Message Header

4.3 Header Reception Operations

4.4 Message Translation

4.5 Message Transmission

4.6 Printing Operations 
The Remittance Processing System is a prototype developed for the Internal Revenue Service to test a new concept in automating the processing of taxpayer remittances. Central to the new processing concept was the integration of the Direct Data Entry System. Initial Entry Station with equipment components that would eliminate a number of individual manual operations, permit operator interface with the central DDES computer, and provide for verification of initial entry, check endorsement, audit trail data, remittance documentation, and affixing information to taxpayer documentation accompanying the remittance. This report describes the equipment which was configured to implement these processes and to demonstrate the viability of the concept.

\subsection{INTRODUCTION}

The National Bureau of Standards participation in the development of equipment for the Internal Revenue Service Remittance Processing System was initiated in August 1971 through the standard interagency agreement process. IRS requested NBS assistance in the development of equipment which would prove the validity of a "new" concept in processing taxpayer remittances. If the concept proved valid, the equipment would provide the basis for development of technical specifications for procurement of Remittance Processing Equipment from industrial sources.

By December 1971, the equipment concept in support of the new processing procedure was agreed upon and design, development, and fabrication of the Remittance Processing System Controller (RPSC) and the Remittance Processing Device (RPD) undertaken. Central to the new processing concept was the integration of the Direct Data Entry System Initial Entry Station with equipment components that would eliminate a number of individual manual operations, permit operator interface with the central DDES computer, and provide for verification of initial entry, check endorsement, audit trail data, remittance documentation, and affixing of information to taxpayer documentation accompanying the remittance. Of prime consideration was the constraint "not to interfere" with the Direct Data Entry System with which the RPS had to interface. Of equal. importance was the accuracy requirement in both data handling and throughout the various print operations. Such constraints influenced the design approach to both the controller and the electronic logic driving the various components of the RPD.

The Controller underwent initial test at the IRS Philadelphia Service Center in mid 1972 and preliminary testing of the RPD and its components was accomplished by December 1972. Design and configuration 
was frozen in January 1973 and installation effected by 30 June 1973. The prototype system passed the IRS acceptance test procedures in late August 1973 and pilot operation was initiated In early September.

The Remittance Processing System is controlled by the Direct Data Entry System (DDES) computer. The DDES computer programs in the Philadelphia Service Center have been modified to:

(1) Accept RPS data through a dedicated Display Controller Unit (DCU) and its associated Key Station Terminals (KST).

(2) Eliminate the requirement for input of Header Check Entry for RPS records.

(3) Generate consecutive serial numbers for RPS payments.

(4) Accumulate RPS block amount and document count totals.

(5) Validate and consistency check RPS data.

(6) Accept new RPS special status message and not allow certain "standard" DDES special messages.

(7) Produce a separate tape for RPS payments at end of each work shift.

The Remittance Processing System Controller (RPSC) is an electronic device designed to provide traffic control and communication functions for the RPS and interface the RPS with the DDES. A single RPSC to control ten RPDs has been provided. Collocated with the DCU servicing the KSTs associated with the RPS, the RPSC operation must not interfere with other operations going on within the RPS, with the DDES or with the DDES computer.

Each RPD includes a DDES keyboard and screen, a check processor (encoding machine with a printing unit), a teletype printer (TTY), a document labeller with printer and a message display panel and control.

The RPD check processor will encode the remittance amount entered by the RPD operator in magnetic ink on the face of the remittance, stamp the U.S. Treasury endorsement on the reverse of the remittance and print an "audit trail" on the reverse of the remittance, 1 " from the document edge. The audit trail will consist of the Social Security Number (SSN) or Employer's Identification Number (EIN), tax period, transaction code and full Document Locator Number (DLN).

The RPD teletype printer is used to automatically print a Remittance Register (Form Sub-813) for each block of payments, which lists header data and payment records consisting of the DLN, SSN, or EIN, tax period and remittance amount. 
The document labeller prints the DLN, SSN, or EIN, and amount of the payment on a label, which is then affixed to the document. The documents are manually stacked by the operator following the labeling process. Numbers printed on the document label are alike in size and color. Print color may be changed by changing the print wheel ink roller.

Section I of this report provides a general description of the modules and functions which are a part of the Remittance Processing System and in particular some of the existing system characteristics which influenced the design of this prototype system. The chapters of this section contain detalled descriptions of the various system componentry. All descriptions reference the proper drawings where appropriate. All system drawings are contained in Section II. 


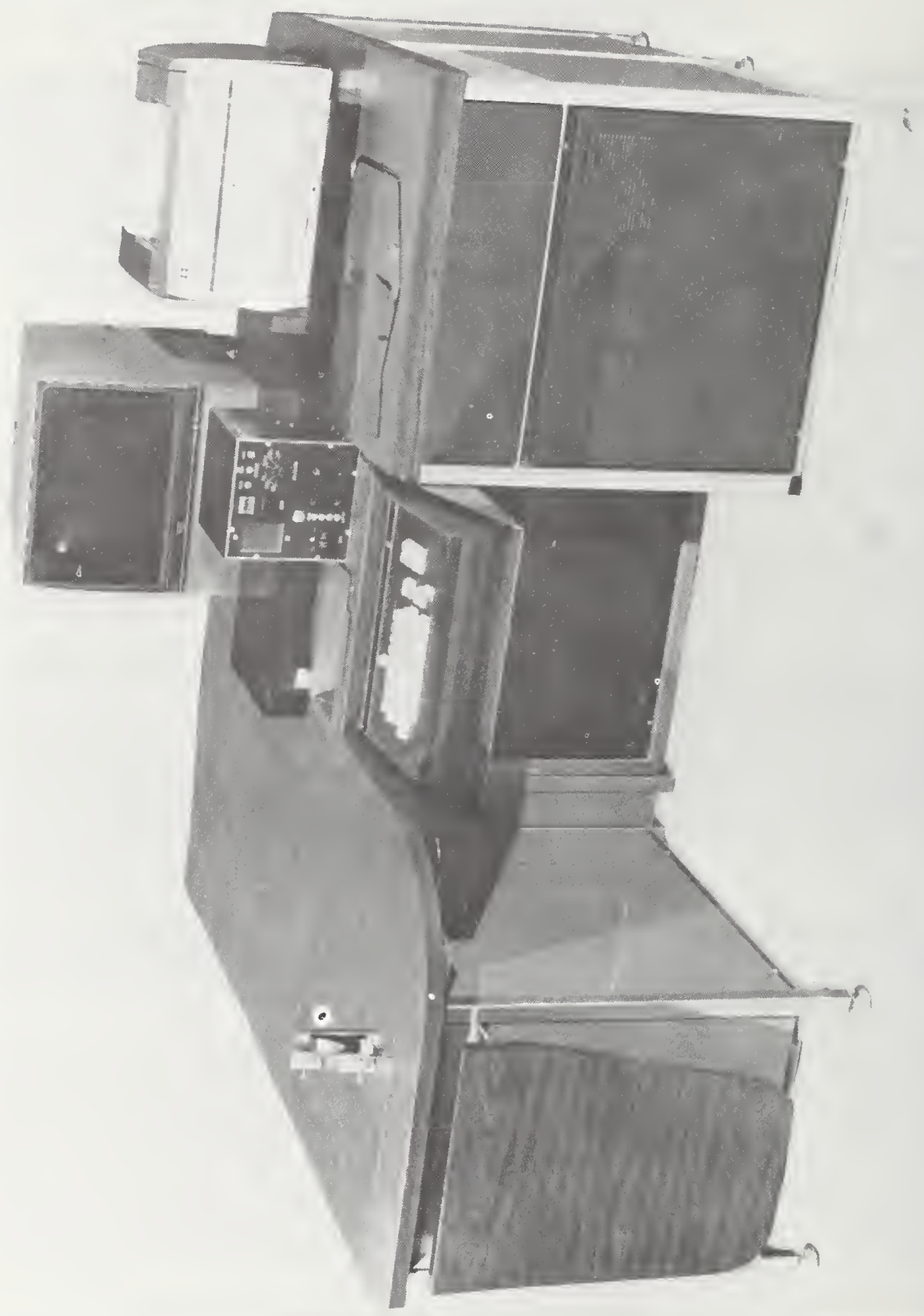

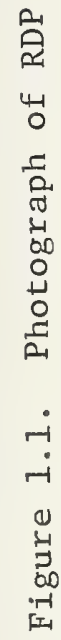




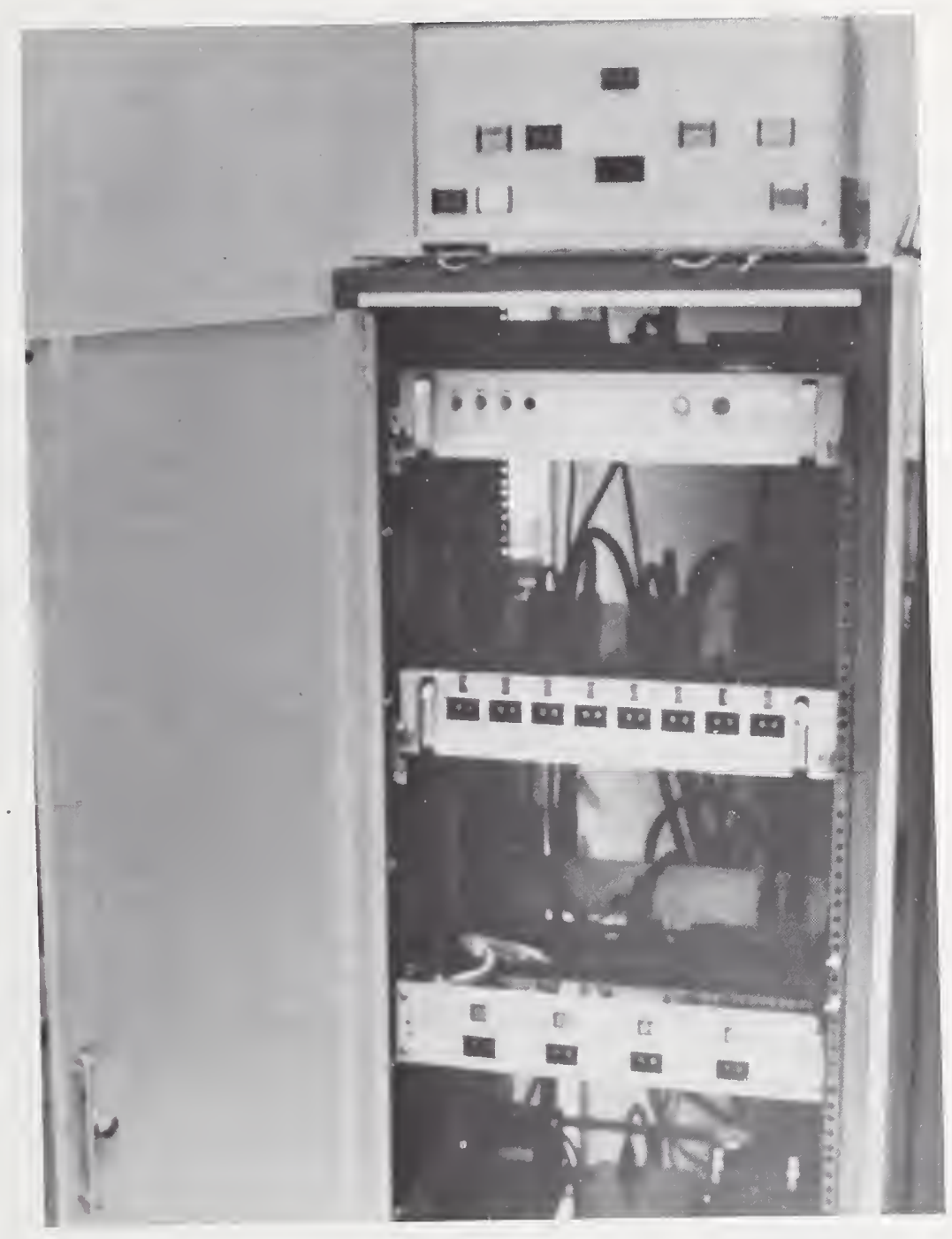

Figure 1.2. Photograph of

RPS Controller 


\subsection{ENVIRONMENT}

2.1 The RPS works in conjunction with the current GE 4020 Direct Data Entry System (DDES). Its operations do not interfere with other operations going on in the system, or with current system hardware. It includes within the Remittance Processing Devices (RPD's) the currently used display and keyboard units (KST's). The KST's incorporated in the RPD's are usually used by Second Entry Operations (SEO) in a Key Verifying (KV) mode only. However, Original Entry (OE) can be made from the RPD station.

2.2 The 10 prototype units, with their associated RPS controller, are installed in the Remittance Processing Area of the IRS Service Center in Philadelphia, $\mathrm{Pa}$, and are operated by remittance area personnel, who are trained in RPS operations. Operational experience and test results will be used to determine the needs for modifications of the prototype units or of the specifications to be derived from them.

2.3 Components of the prototype units for the most part are assemblies of available commercial units or were built by NBS.

2.4 The RPD units and their associated Remittance Processing System Controller (RPSC) are installed in the Remittance Processing Area. This is a room approximately $70 \mathrm{x} 96 \mathrm{feet}$, having a raised floor, air conditioning and power distribution typical of a site for computer installation. The ten prototype RPD's occupy approximately one third of the floor space.

\subsection{SYSTEM COMPONENTS}

\subsection{General}

The RPS, as presently conceived and constructed is described below, and is shown in block diagram form in Figure 3.1. It consists of a single RPSC and up to ten RPD's. No inferences should be drawn from the size, shape, or location of the component blocks of the RPD shown in Figure 3.1. Associated with each RPD is a KST unit to be used by the same operator, and mounted on the RPD. The two units are electrically and functionally independent. The KST units use a common DCU which share the line to the Communication Coupler (CC) used by the RPSC.

\subsection{The RPSC}

The RPSC consists of electronics built by NBS to provide 12 


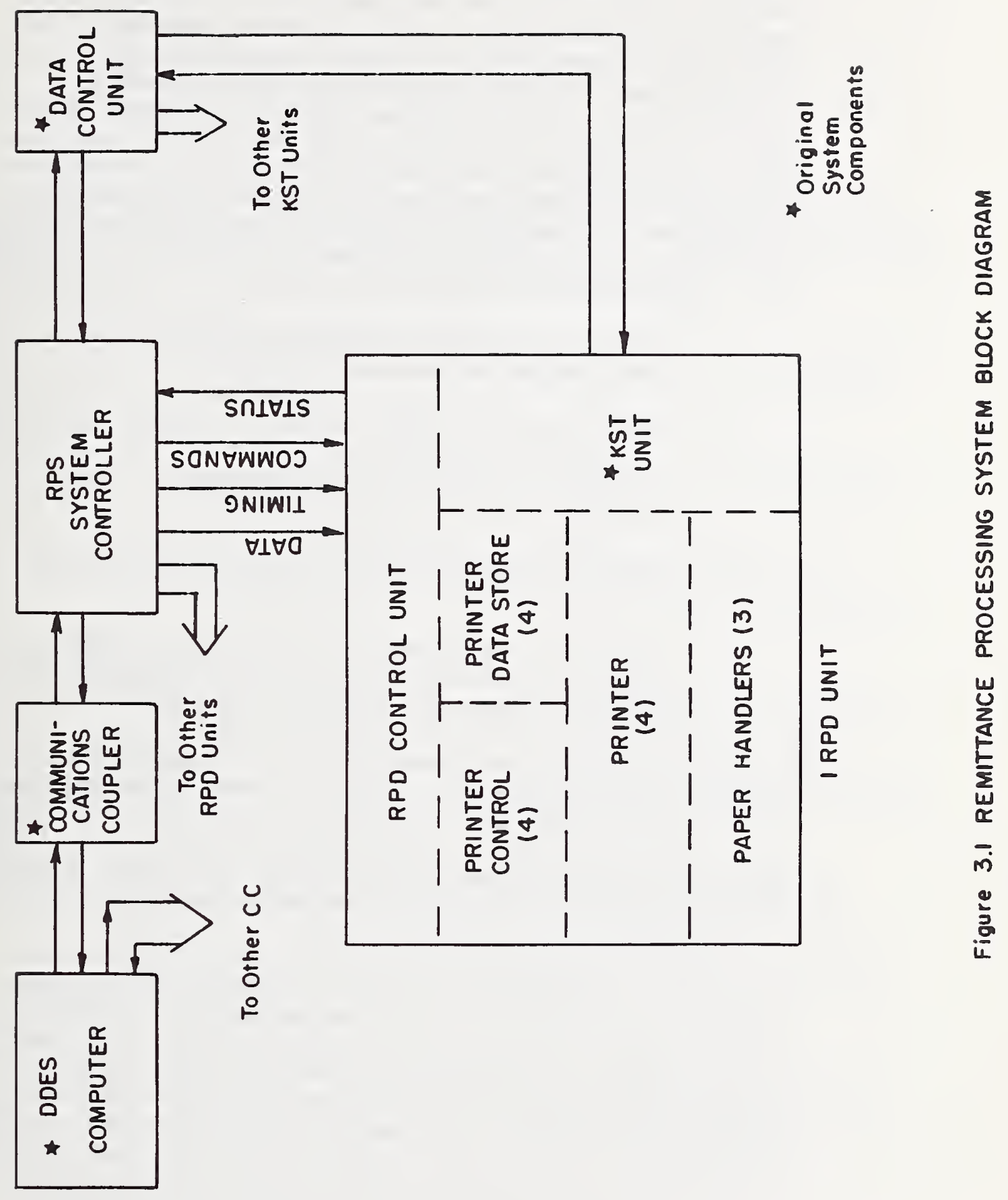


traffic control and communications functions for the RPS. It is collocated with the DCU servicing both the KST's mounted on the RPD's for $S E$, and those providing the $O E$ functions related to the remittance input. Because the line to the Communications Coupler is shared with the DCU, the RPSC must provide a priority system for message transmission. This is a first-come, first-served system wherein if either controller seizes the line it will hold it until its complete message queue has been transmitted. The possible delays caused by this system represents the total direct interaction between the two subsystems outside the computer. The other functions of the RPSC are concerned with communications to and from RPD units. They include:

a. Routing of message elements to proper storage locations in the proper RPD units;

b. Making certain format checks on incoming messages;

c. Assembling and transmitting messages in reply to messages received from the computer;

d. Ignoring messages for RPD's under predetermined conditions;

e. Inhibiting the reception of new messages when failure to transmit messages to the computer has caused too large a backlog to develop.

The interfaces between the RPSC and the Communications Coupler (CC) and DCU are designed to meet the requirements of EIA Standard RS-232B. When power is off, a set of relay contacts are used to bridge across any RPSC gating in the RS-232B signal or control lines. This restores the DDES to its original condition and permits operations independent of the RPSC.

To prevent electrical interaction between this existing system and the RPS, the interface is made up of LED/LSD units. These provide a light energy interface, rather than an electrical one. Those circuits which must be powered from the DDES side of the interface are supplied from power supplies obtaining their energy from the same 115 VAC Iine as the DCU; those circuits which must be powered from the RPS side of the interface have power supplies obtaining their energy from the same 115 VAC line as the RPDs.

\subsection{The RPD}

\section{3 .1}

As shown in Figure 3.1, the RPD is made up of several subunits. The electronic portions consist of the RPD control 
unit, a printer control unit for each of the three printers, a data storage unit for each of the four print operations, and a console for operator intervention when required. Some of these units have been constructed at NBS, others were provided with the various printers by their manufacturers. There are four controlled print operations in the system provided by three printers:

a. Printing an audit trail on the remittances, which is accomplished during the first pass through a Burroughs Model S-102/S-103 Electronic Proof Encoder. (The S-102/S-103 is the basic element in the design of the prototype RPD station);

b. Printing remittance amounts, which is accomplished by a second pass through the S-102/S-103 Encoder printer;

c. Pringing Document Labels, which is accomplished on a modified Computer Terminal Systems (CTS) strip printer; and

d. Printing Register, which is accomplished on a Model 37 RO Teletype with some minor modifications made by NBS.

The MICR Encoder and Audit Trail Printer share a common paperhandling system. A mechanical system causes the check to loop back to the start of the remittance slot in a position to print the amount on the face of the remittance. Endorsing and stacking then takes place after the two print operations.

The document labeller does not presently incorporate a document handler to stack the documents automatically. Instead, the operator places the document into the device which prints a label with the proper text and attaches it to the document; the operator then removes and stacks the labelled document.

The Register paper handler will feed continuous pin feed forms to the Teletype unit, and stack the output web in a convenient tray located at the rear of the printer.

\subsubsection{RPD Functions}

Each RPD performs the following functions:

a. React only to messages selected for 1t from the message stream by the RPSC:

b. Carry out certain format checks on incoming messages; 
c. Indicate to the RPS certain status conditions;

d. Request transmission to the computer of messages concerning reception and reaction to messages received from the computer;

e. Print the transmitted texts as prescribed in the messages from the computer;

f. Signal to the operator any abnormal conditions;

g. Respond to manual intervention by the operator in case of malfunction, or under other special circumstances.

The RPD units are presently connected in star fashion to the RPSC by cables which do not meet RS-232B standards. A connector determined priority system controls the order of message transmission by the RPSC for the RPDs.

An RPD.does not interfere with the DDES system directly, since it sends only status signals back to the RPSC. Filtering and other methods of suppressing radiated signal interference and electrical line interference have been used to prevent indirect interference with the DDES.

\subsection{SYSTEM FUNCTIONS}

\subsection{Message Reception}

\subsubsection{Message Formats}

a. Information - The message from the computer to the RPD must follow the format shown in Figure 4.1. The RPSC will check for the occurrence of exactly four printer command flags, and the presence or absence of certain control codes. If no valid RPD address has been received, an error flag will cause the message to be ignored. Once a valid RPD address has been received, an error flag will cause a NAK message to be generated. In either case, no printing will be done.

b. Code Formats - Messages from the computer are sent as 7-bit ASCII codes with odd parity in a synchronous mode, least significant bits first. These must be converted to the appropriate codes and formats to drive the individual printers. This is done in the RPD printer control units. Storage of the data varies in format depending upon the printer and the manufacturer of the storage unit. The RPD unit checks lateral parity (odd) of each character as received, and the longitudinal 16 


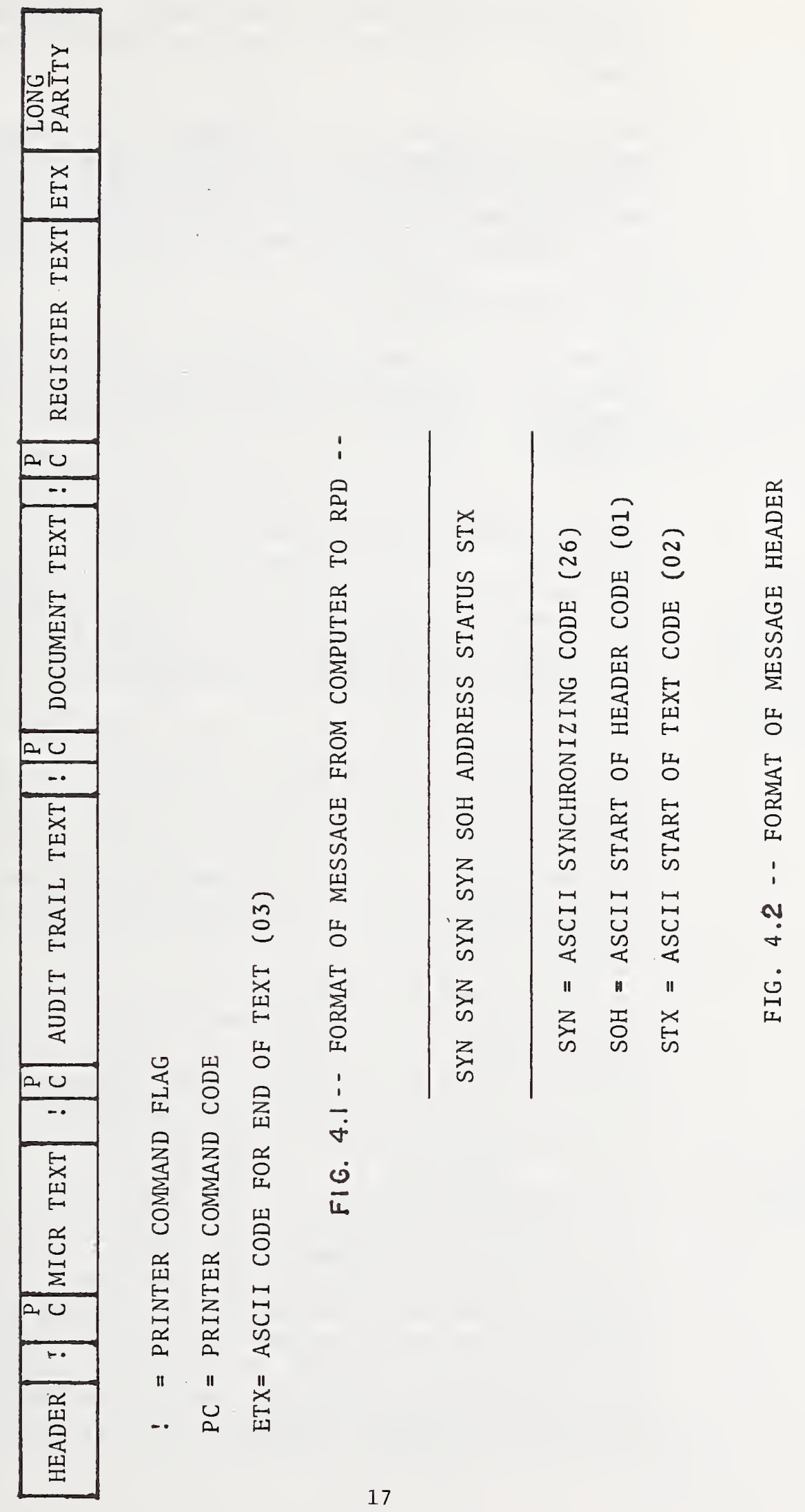


parity (even) generated by the RPSC. Thus transmission is checked from source to destination. Read-back from a printer is not used to check the actual character printed.

c. Electrical Formats - The RS-232B standard signal levels are used from computer to the RPSC and from there to RPD units. Within the RPD, signals vary with the printer and electronics being used. In general, internal signals are referenced to ground and not balanced about ground.

\subsubsection{Header Translation}

a. Header Format - The common header format is shown in Figure 4.2. Any of the current status codes is acceptable, the expected one being NUL for incoming messages.

Address codes must have bits 6 and 7 equal to 1 in order to be accepted by the DCU. The DCU will reject all others. Bit 7 must be a 1 and bit 6 must be a zero for the RPSC to accept the address as an RPD address. All others will be rejected. It is by this means that the messages for the RPD's are separated from those for the $\mathrm{KST}^{\prime} \mathrm{s}$ in processing the message stream.

The five minor bits of the address for an RPD are set up in switches on the RPSC. There is a set of switches for each RPD, so that any unit may be set up to any address. It should be noted that since the RPD address is associated in the RPS program with the KST having an address differing from it by one, it is necessary to re-plug the KST unit on the RPD into the proper fack on the DCU whenever the RPD address is changed.

An RPD address is not recognized as being valid, if (1) there is no unit with switches set up to the incoming address code, (2) if such a unit is not operating, or (3) if its ACK message indicating completion of printing has not yet been transmitted to the computer. A message with an invalid RPD address is ignored.

b. RPSC Operation (Figure 4.3) - The RPSC operations are divided into eight phases, the first five of which are associated with processing the message header. The figure illustrates these first five phases. Note that in $\emptyset 3$ the address is examined and compared to the RPD addresses set up in the RPSC switches. Should a valid RPD address not be found, the operation reverts to $\emptyset$, and the message is ignored. Once an address is accepted as valid, some reply message will be generated at the 


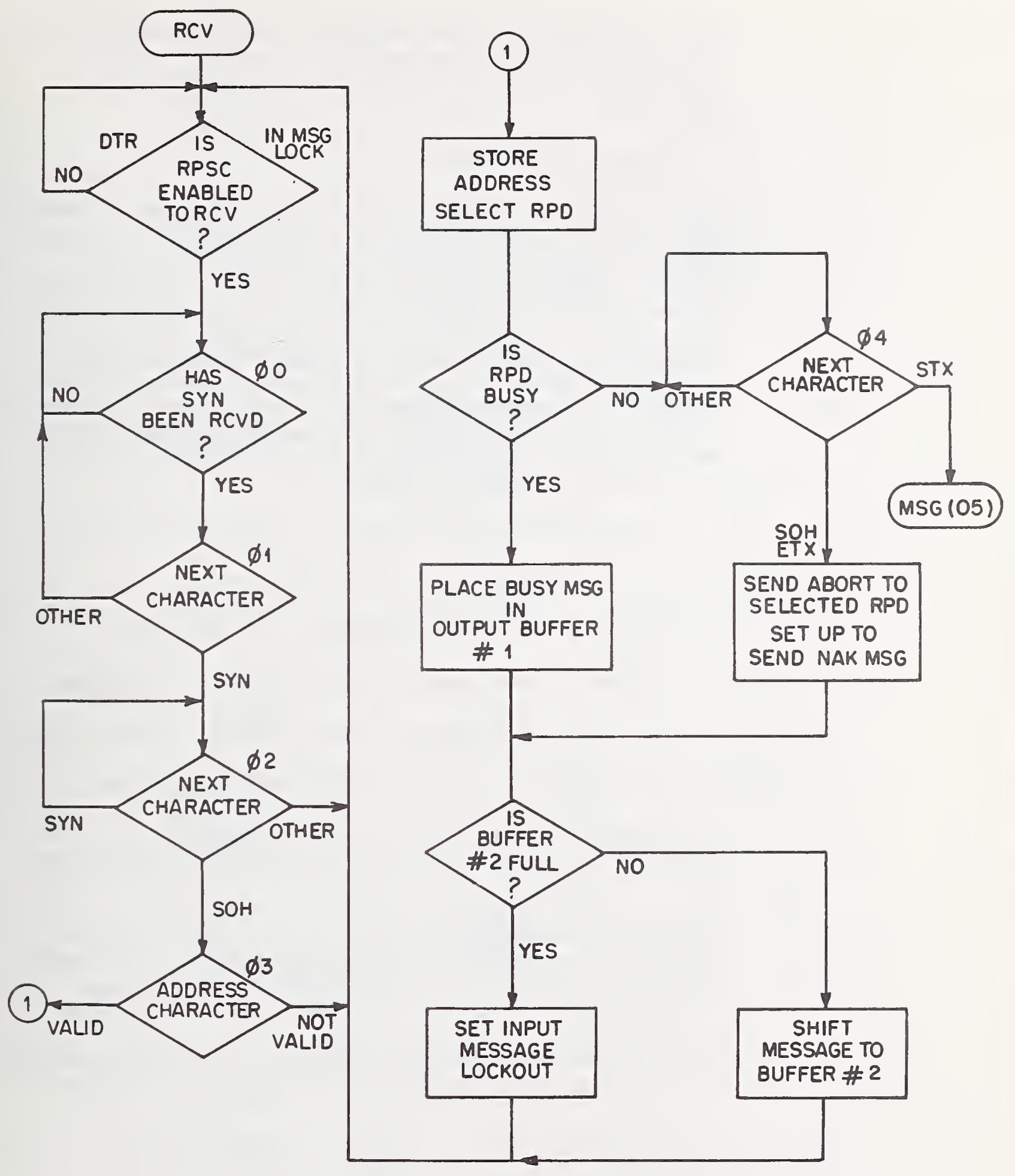

Figure 4.3 HEADER RECEPTION OPERATIONS 
end of reception of the message. The addressed unit will be selected for command and text transfer. Header translation normally ends with the recognition of an STX code.

\subsubsection{Message Translation}

a. Printer Controls (Figure 4.4) - Upon receiving a Command Flag character, the RPSC will count it and will command the selected RPD to accept the next character as a printer command, which will also be used to select the proper buffer for the string of characters which follow it. These will be taken as text for that printer. While there is a set order of message sections in the prescribed format, the circuitry will operate with any sequence as long as Register Text is last. An erroneous control character will not only cause the loss of the message desired to be printed, but may also substitute it for one previously received.

The printer command characters (ASCII codes) are: Q = non-print, even if text follows;

$A=$ MICR printing is desired, text follows;

$B=$ Audit Trail printing is desired, text follows;

$D=$ Document printing is desired, text follows; and

$\mathrm{H}=$ Register printing is desired, text follows.

An illegal command character will be discovered by the RPD and will cause an abort and NAK message generation.

Upon the reception of a valid Print Command code, the characters following it will be loaded into the data store for the printer selected by the code. If no text is provided between the print command and the next Command Flag, the data store will contain all zeros.

b. The MICR Message - The MICR message sent from the computer will consist of a maximum of 13 characters as follows:

1 Command Flag ! 041

1 Print Command e $100^{8}$

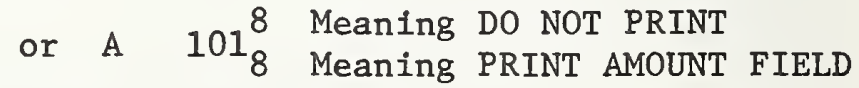




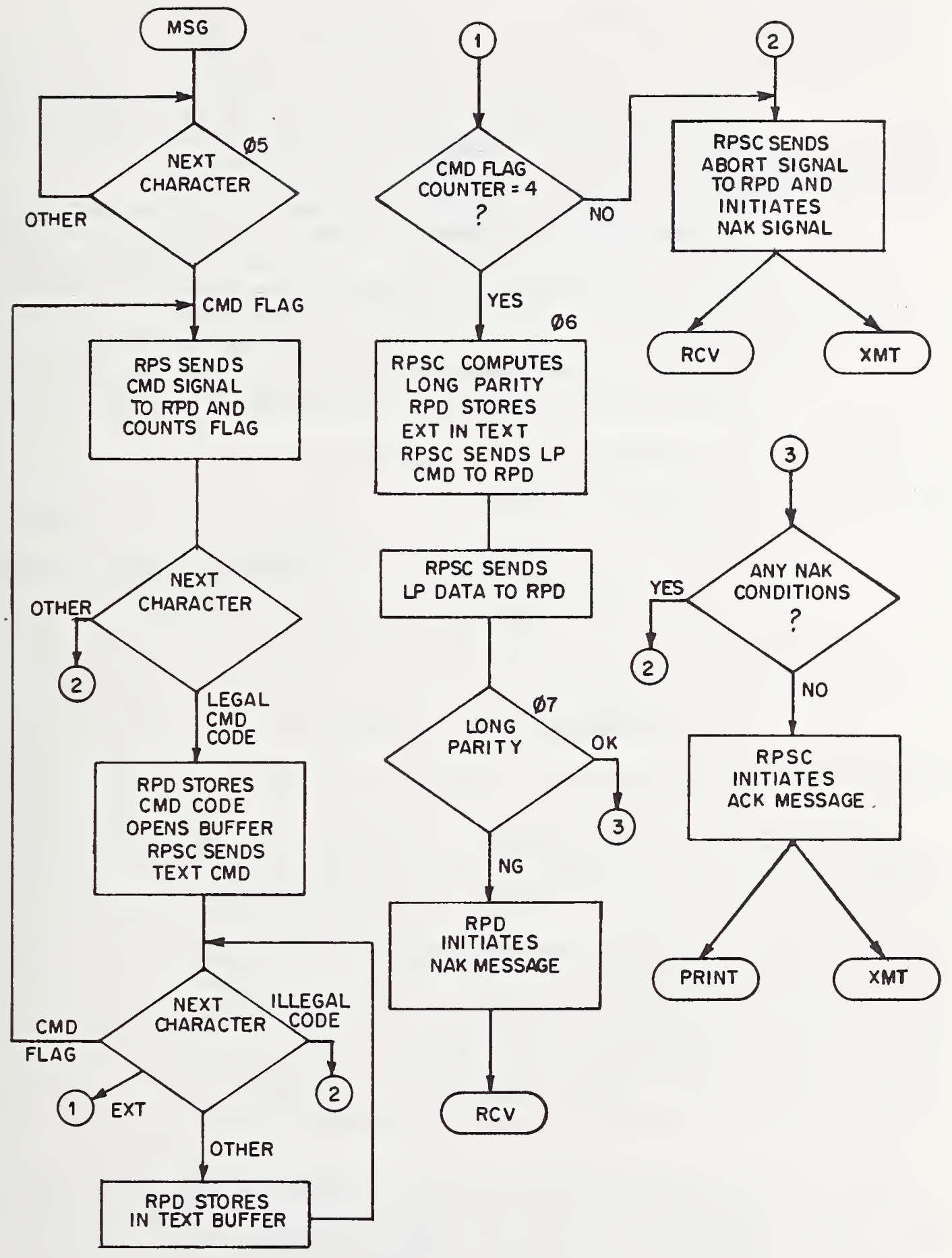

Figure 4.4 MESSAGE TRANSLATION 
0 to 10 decimal digits representing the remittance amount

1 Breaker

$$
+\quad 053_{8}
$$

The MICR printer will print 12 characters: the amount filled with leading zeros up to 10 digits, preceded and followed by the MICR Amount Symbol.

Should an A Print Command be sent with no amount entered, the printer will print 10 zeros preceded and followed by the MICR Amount Symbol. Any amount entered after a (a) Print Command will be ignored, and nothing will be printed.

c. The Audit Trail Message - This is a fixed-length message of 38 characters as follows:

1 Command Flag ! $041_{8}$

1 Print Command a $100_{8}$ Meaning DO NOT PRINT

or B ${ }^{102} 8$ Meaning PRINT AUDIT TRAIL

1 Pointer Symbol ; $073_{8}$

9 Numerics of the TIN

1 Spacing Symbol ? $\quad 077_{8}$

4 Numerics of the $T / P$

1 Spacing Symbo1 ? $077_{8}$

1 Pointer Symbol; $073_{8}$

3 Numerics of the $\mathrm{T} / \mathrm{C}$

1 Spacing Symbol ? $077_{8}$

14 Numerics of the DLN

1 Pointer Symbol ; $073_{8}$

The Pointer Symbol and the Spacing Symbol were initially required because of the possible use of two different Audit Trail Printers having different symbols and codes. The Spacing Symbol will result in a blank space with either printer as it is used as a Print Inhibit code. There is no common symbol suitable for use as a pointer in the two printer repertories. When the MICR encoder 
printer is used, this Pointer Symbol code will cause the MICR Transit Number Symbol to be printed.

The MICR printer prints all 36 characters following the Print Command in a single line. The message is printed upside down on the back of the remittance within $1 / 2$ inch of the top.

Should a B Print Command be issued with no text between the command and the next Command Flag, a printout of 36 (or 34) zeros will result. Should text follow a c Print Command, it will not be printed.

d. The Document Message - This will be sent from the computer as a varlable-length message with a maximum of 38 characters:

1 Command Flag ! $041_{8}$

1 Print Command @ $100_{8}$ Meaning DO NOT PRINT

14 or $\quad \mathrm{D}{ }^{104} 8$ Meaning PRINT DOC MSG

1 Space $\quad 040_{8}$

9 Numerics of the TIN

1 Space $\quad$ b $\quad 0_{8}$

0 to 10 Numerics of the Amount

1 Breaker Symbol $+053_{8}$ Meaning END OF AMOUNT

The Text is printed (except for the + ) just as sent. Leading zeros are not supplied for the amount. Should a D Print Command be issued with no text, a message of 36 zeros will be printed. If only the +1 s sent, a message of 26 zeros will be printed. Should a @ Print Command be issued, any following text in this document message will not be printed. It should be noted that this printer is capable of alphanumeric output, corresponding to the codes in the first four columns of the graphics in the ASCII table. The printed label is 35 characters long including spaces.

The Document Message is printed upon a $1 / 2 \times 4$ inch heated pressure-sensitive label which is attached to the document within 1 - inch of a reference edge. Its position along this edge is determined by manually settable guides on the label attaching device. A label is being used because of the difficulties of directly printing upon a document, such as the Form 1040, which 
may vary in thickness across any single document as well as from document to document, and where the presence of tape or staples may affect the printer or the printing.

e. The Register Message - This is sent from the computer as a variable-length message with a maximum length set by the storage available for a KST unit and the amount of such storage taken up by the other printer messages. Its format is as follows:

1 Command Flag ! $\quad 041_{8}$

1 Printer Command e $100_{8}$ Meaning DO NOT PRINT or $\mathrm{H} \quad 110_{8}$ Meaning PRINT REG. TEXT

A variable number of alphanumeric and printer control codes

1 End of Text Code ETX ${ }^{003} 8$

The ETX code is that required to indicate the end of the entire computer message, and not an additional one (See Fig. 4.1). Should no text be provided with an $\mathrm{H}$ Print Command, nothing will be printed and no printer control operations will occur. Should text follow a @ Print Command, nothing will be printed and no printer control operations will occur.

The Register printer is a serial device, and responds to codes sent it in order of arrival. Any printer control commands, such as ribbon color change, must be sent at the proper time in the code stream. While some commands, such as Form Feed, take more than one character time, there is no need to supply fill characters, as the interface circuitry will supply the required delay of the next code sent to the printer. Additional circuitry automatically supplies a Form Feed code when printing would occur within 1/2- inch of the bottom of an 11inch sheet. Top of Form is set at 1/2- inch from the top of the sheet. A single set of horizontal tabulation stops are set up by the maintenance engineer according to a prescribed 1ist (See Section $V$ of this report). The Horizontal Tab Clear and Horizontal Tab Set functions are then mechanically disabled to prevent accidental clearing or setting of additional tab stops. Should the IRS programmers wish to change the tab stop positions, $t$ the maintenance engineer must provide the new set-up. There is no provision to clear and set tabs by computer message. Use of vertical tabulation codes except Form Feed is forbidden to the programmers. 
The ribbon color control codes are used to permit the printing of certain information on the copies, but not on the original of the register form. Special ribbons with uninked portions where red ink is normally used have been provided.

The Register Form, SUB 813, is printed as part of the block processing, and resembles the Form 813 now in use in the manual procedure. It consists of a header, which gives block information and column headings; a body, which will consist of a line entry for each remittance; and a tail, which will consist of summary data and labels for them. It may be variable in length, and usually will consist of two or three 11-inch sections. Each section is fed as a lined sequentially numbered 11-inch perforated sheet in roll or fanfold form, in three or more plies, as required. In case a section should accidentally be detached from the rest of the form, it may be properly mated by use of the sequential numbering.

\subsubsection{End of Message Events (Figure 4.4)}

When the RPSC receives an ETX code, it develops the longitudinal parity (LP) character and sends it to the RPD instead of the LP character which may be attached to the message as it is received from the computer. If no LP character is sent by the computer, no harm will be done. The command flag count will also be checked. If it is bad, a NAK and an abort will occur. Otherwise, the RPSC will return to the $R E A D Y$ state.

When the RPSC receives an ETX code, it signals the RPD to accept the next character as the longitudinal parity character, generate it, and load it into the input data buffer. The RPD will suspend lateral parity checking, compare the longitudinal parity character with its own, and if the two do not match, signal the RPSC to send a NAK message. Further processing of the message will be aborted. If there is no reason to send a NAK message, the RPSC will send an ACK message, while the RPD proceeds with the printing cycle. At the end of the printing cycle, the RPD will signal the RPSC to send a second ACK message.

\subsection{Message Transmission (Figure 4.5)}

\subsubsection{Format}

The format for all messages transmitted to the computer is the same as that of a received message, except nothing lies between STX and ETX. Only three codes are acceptable in the status character: ACK, NAK, and BSY (busy). The 


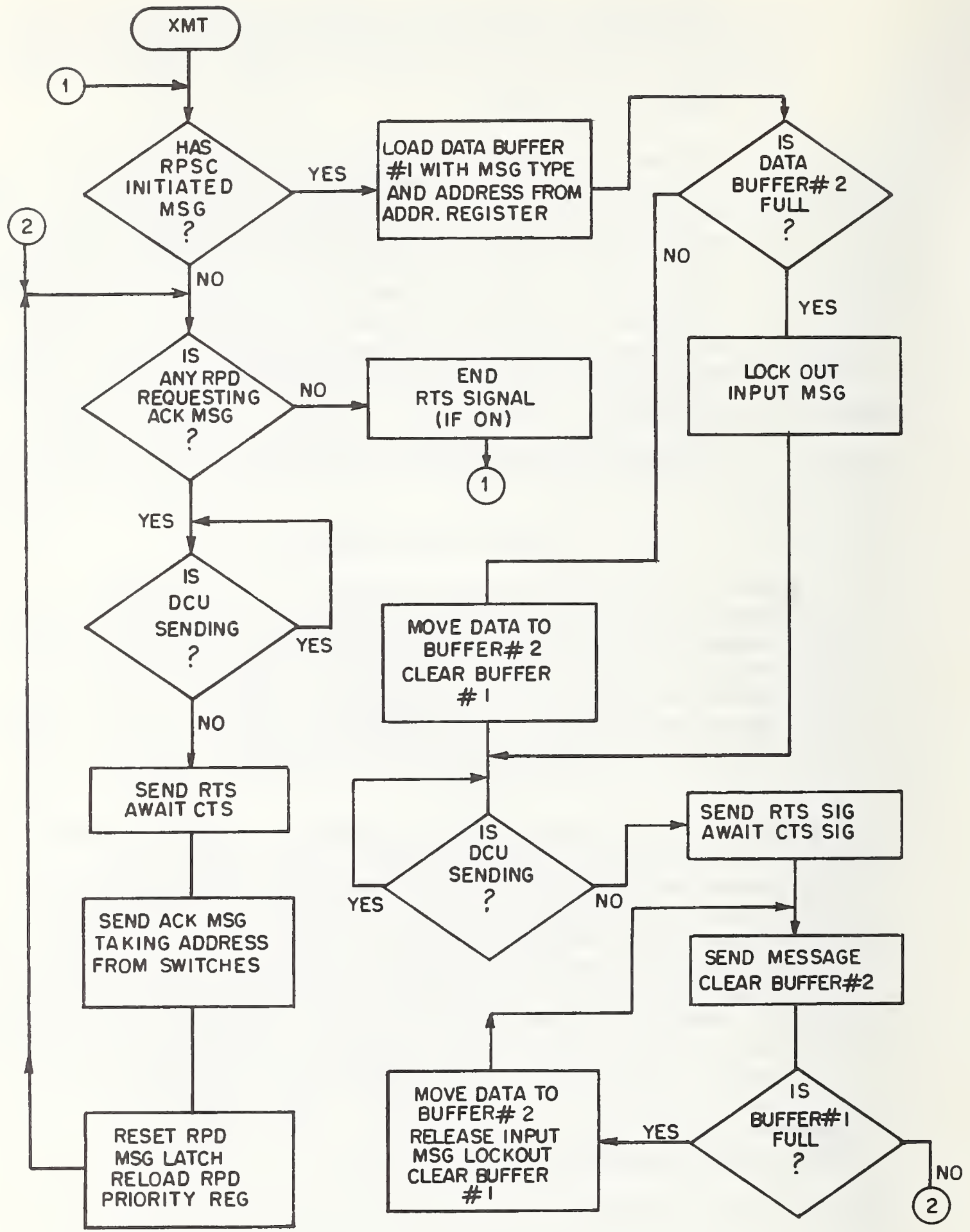

Figure 4.5 MESSAGE TRANSMISSION 
BSY is not expected to occur normally in the planned use of the system.

In order to match current practices, the LP character is sent as all $1^{\prime}$ 's.

\subsubsection{Data}

The data for the changeable characters of the message consists of the RPD address and the message type. To handle the message type, a message of each type is stored in the RPSC Read Only Memory (ROM). The address is supplied from either the RPSC address buffer (for a reply to a current message) or from the address switches associated with the RPD units (for ACK messages at the end of the printing cycle). Because the current DDES system will not accept a message otherwise, reply messages all have bits 6 and 7 of the address set to $1^{\prime} \mathrm{s}$. This also provides for buffer areas in the computer. KST's on the same DCU line as the RPD's cannot have these addresses.

\subsubsection{Transmission}

When a message is in its output buffer, the RPSC will attempt to seize the transmission line. If the DCU is using it, the RPSC must wait. When the RPSC does seize the transmission line, it will continue to assemble and transmit all messages which are awaiting assembly and transmission before releasing the line.

\subsection{Printing (Figure 4.6)}

\subsubsection{Sequencing}

If, at the start of the printing cycle, a remittance printing operation is required, but no remittance has been provided, no printing will be initiated. After a short time delay, a signal is given to the operator to correct this discrepancy. Once the remittance has been provided, all requested printing operations go on simultaneously and independently.

Should document printing be required, and there is no document in position, visual and audible alarms are set for the operator, and all other requested printing takes place.

At the successful completion of each printing operation, a control flag is reset. When all are reset, an ACK message is initiated and the RPD returns to the READY state. Should they not all be reset, the unit will remain in a BUSY state until human intervention takes place. An alarm for an overlong BUSY state is not provided as this condition can be 


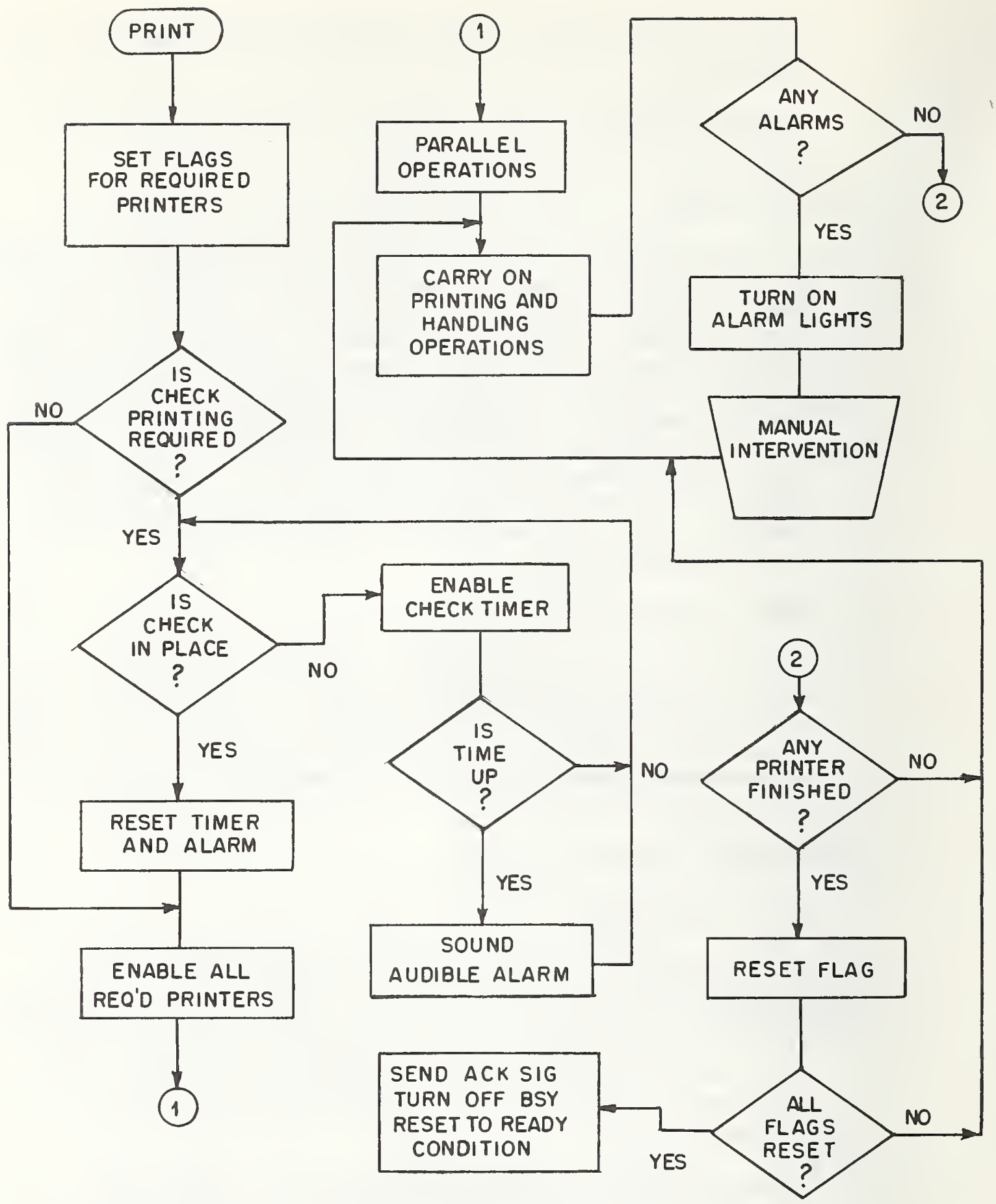

Figure 4.6 PRINTING OPERATIONS 
discovered from programmed interlocks with the associated KST.

\subsubsection{Operational Checks}

In all paper handlers, feed checks are made to ascertain that the paper is moving properly and eventually end's up in the stacker bins. Should a jam occur, a warning light is lit for the operator, and the RPD will remain in a BUSY state until the jam is cleared.

All the printing devices are monitored similarly for failure to be in a proper operating condition. The end of the MICR printer ribbon or a break in the tape will cause an alarm. Fallure of the Register Printer (TTY) to provide a new line for each message, or the occurrence of low form supply will also cause an alarm. A Paper Out signal is provided for the document label printer.

\subsubsection{Operator's Panel}

To provide the operator with necessary information and control, an operator's panel is provided. This incorporates general condition lights (Green $=$ Go, Amber = document not in place, Red = Malfunction Alarm); an audible tone generator to warn that a remittance or document has not been placed in proper position; a series of messages to indicate what is wrong, so that the operator may either clear a $\mathrm{jam}$, supply a need, or call a maintenance engineer; and a set of manual control switches so that the unit can recover after a malfunction alarm by continuing from where the operation stopped, repeating the particular printing operation, or clearing out the storage and control for that printer in preparation for other recovery procedures. Such manual printing control is possible since each printer has its own message buffer, which is not cleared until a new message is to be entered or the reset switch is operated when in the manual mode. 
The Remittance Processing System Controller

\section{Table of Contents}

1.0

1.1

1.2

2.0

2.1

2.2

2.3

2.4

2.5

3.0

3.1

3.2

3.3

4.0

4.1

4.2

4.3

4.4

5.0

5.1

5.2

6.0

6.1

6.2

6.3

6.4

6.5

6.6

6.7

6.8

7.0

8.0

9.0

9.1

9.2
Introduction

Background

Environment

RPSC Functions

General

The Transparency (RPS-DDES Interface)

The Received Message Handler

The RPD Control (RPD Interface)

The Reply Unit (Transmitted Message Handler)

The Transparency

Physical

Electrical

Electronics

Data Flow

Physical

Electrical

Message Processing

The RPD Interface

The RPSC Control Circuitry

Physical

The RPSC Control Panel

The RPSC Reply Circuitry

Physical

The Transmitted Message Handler (TMH)

Transmitted Message Format

Message Initiation

Message Priority

Transmitted Message Storage

RMH Lockout

Message Trasnmission

The Power Supply

The Transparency Drawer

RPSC Detailed Description of Operation

RPSC Operation

Sequence of Operations in RPSC 
9.3 Operation of RPSC Major Components

9.4 Recelpt of a Message from the Computer

9.5 Generation of Second Acknowledgement

9.6 RPSC Annex

10.0 RPSC Detalled Description of Logic Circuitry

10.1 Data Flow (Drawings 2.2.1 - 2.2.7)

10.2 RPSC Control Circuits (Drswings 2.3.1 - 2.3.7)

10.3 RPSC Reply Circuitry (Drawings 2.4.1 - 2.4.6) 


\section{List of Figures}

$\begin{array}{ll}3.1 & \text { Transparency Drawer Component Layout } \\ 3.2 & \text { RS-232 Cable to Computer } \\ 3.3 & \text { RPSC Inter-drawer Cable } \\ 3.4 & \text { Integrated Circuit Layout RPSC Transparency } \\ 4.1 & \text { RPSC Electronics Drawer } \\ 4.2 & \text { RPSC Annex Electronics Drawer } \\ 4.3 & \text { Message Format, Incoming } \\ 4.4 & \text { Message Text Formats } \\ 4.5 & \text { RPD to RPSC Cable } \\ 5.1 & \text { Clock Timing Diagram } \\ 5.2 & \text { RPSC Phase Counter Timing } \\ 5.3 & \text { RPSC Control Timing Diagram } \\ 7.1 & \text { RPSC Power Supply Drawer } \\ 9.3 & \text { RPSC Overall Operation }\end{array}$




\subsection{Background}

The Remittance Processing System Controller (RPSC) is an electronic device designed to provide traffic control and communications functions for the Remittance Processing System (RPS). Its general functions were described in Section I of this report. A single RPSC to control ten Remittance Processing Devices (RPD) has been provided. Original plans for the RPSC called for it to provide for up to eight RPD's; however, provisions were made in its design to extend its capacity to sixteen RPD channels. It was constructed with twelve channels for the ten RPD system to provide two spare channels. A second drawer in the RPSC cabinet contains the added channel controls, cables and the addition of the necessary interconnections to the original chassis drawer.

\subsection{Environment}

The RPS (Figure $3.1 \mathrm{Sec} .1$ ) is designed to work in conjunction with the GE 4020 Direct Entry System (DDES) presently used in IRS. Its operations must not interfere with other operations going on in the system, or with current system hardware. It is located at the Philadelphia Service Center of the IRS, in an air-conditioned office environment. A dedicated power line serves the units, just as the DDES has a line dedicated to its uses.

\subsection{RPSC FUNCTIONS}

\subsection{General}

The RPSC provides traffic control and communications functions for the RPS. It is collocated with the Device Control Unit (DCU) serving key stations (KST) of the DDES, some of which are located at the RPD's. Since the communications line between the Communications Coupler (CC) must be shared by the DCU and the RPSC, a priority system must be included to prevent interference between messages from the two units. As the interface between the DDES and the RPS lies within the RPSC, isolation between the two systems is provided here.

The other functions of the RPSC are concerned with communications to and from the RPDs. They include:

a. Routing of messages to the proper RPD;

b. Routing message elements to the proper data storage or control storage portions of the RPD; 
c. Making format checks on incoming messages;

d. Making lateral parity checks on incoming messages, and generating longitudinal parity as an added check for transmissions between RPSC and the RPD;

e. Assembling and transmitting messages in response to messages received from the computer via the $\mathrm{CC}$;

f. Rejecting messages under certain conditions.

\subsection{The Transparency}

The Transparency is that portion of the RPSC which provides the interface between the RPS and the DDES. Its functions are to provide:

a. Isolation between the DDES and the RPS electrically, while communicating with the $\mathrm{CC}$;

b. Priority control of the communications from the DCU and the RPS to the CC;

c. Timing signals (Transmitter and Receiver Clocks) when the DCU which normally provides them is not on line.

d. Conversion between the RPSC electronics logic levels and the signal and impedance levels required by EIA RS-232B standards. The communications line to the CC meets these standards, as does the output of the DCU.

\subsection{The Recelved Message Handler}

The Received Message Handler recelves all messages sent from the CC and picks out those meant for the RPDs. It then provides the following functions:

a. Checks message format--a fixed format, variable length message is used in all communications;

b. Provides the information required by the RPD Interface;

c. Removes characters from the message string which are not meant to be included in the printed messages output by the RPDs;

d. Checks lateral parity as the message is received and provides longitudinal parity for messages to the RPD; 


\subsection{The RPD Interface}

This unit controls the transmission of messages to the RPDs. It selects the proper RPD and directs portions of the incoming message to the proper RPD memory or to its memory selection control. It can also indicate an aborted message to the RPD. It receives and acts upon status messages from the RPD.

\subsection{The Transmitted Message Handler}

This unit, on the basis of signals from the RPDs and the Received Message Handler, assembles and transmits various replies to messages from the CC. If transmission of such replies overloads it, it can block off the input to the Recelved Message Handler.

\subsection{THE TRANSPARENCY}

\subsection{Physical (Figure 3.1)}

The Transparency is contained in the top drawer of the RPSC. The lefthand side contains the $+12 \mathrm{~V}$ and $-12 \mathrm{~V}$ DC supplies required by the $\mathrm{RS}-232 \mathrm{~B}$ standards; the right-rand side contains the $+5 \mathrm{~V}$ DC supply required for the internal electronics, the bridging relay and its driver; and the center section contains the local clock controls and the Test Switch in the front portion, and the Transparency logic electronics in the rear. All cabling to other RPSC drawers, and input and output to the $\mathrm{CC}$ and $\mathrm{DCU}$ are at the rear of the drawer.

\subsection{Electrical}

\subsubsection{Isolation}

To provide isolation between the RPS and the DDES, all signals across the boundary between the Transparency and the rest of the DDES are transmitted through optical couplers. Those parts of the isolators on the RPSC side of the boundary are driven from power supplies powered by by the RPS-dedicated line. Those portions on the DDES side are driven by power supplies powered from the DDES-dedicated line. Care must be taken to insure this isolation is maintained whenever the RPSC is connected into the 120 VAC $1 \emptyset$ power lines. The plugs used to connect to the subfloor outlets are of different types to assure proper connection. 


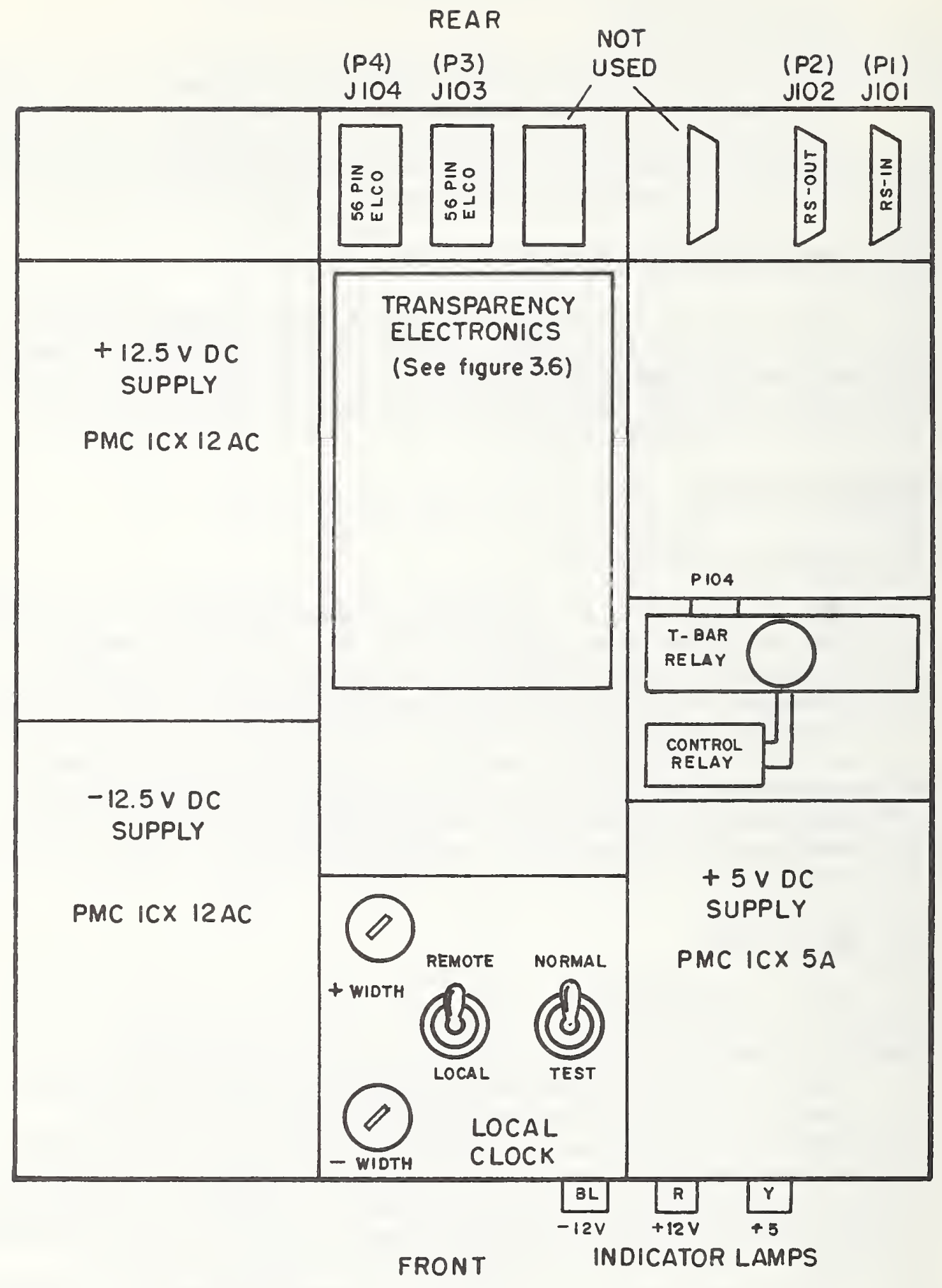

Figure 3.1 TRANSPARENCY CHASSIS-TOP VIEW 
a. To the CC - The cable from the CC is provided as part of the DDES. It terminates in a standard RS-232B male plug, which is inserted in the RS IN connector (J101) at the rear of the chassis. See Figure 3.2

b. To the DCU - This cable is about 10 feet long and terminates at both ends in an RS-232B plug. It differs from the CC-DCU cable in that there is no crossing of leads within the cable. It plugs into the RS OUT socket at the rear of the chassis (J102).

c. The Bridging Relay - The leads to the bridging relay contacts are mostly brought out to an ELCO 56-pin connector (P104) which is plugged into J104 at the rear center of the chassis. For wiring, see drawings numbers $2.1 .1,2.1 .2,2.1 .3,2.1 .4,2.1 .5$, and 2.1.11.

d. Connection between the Transparency and the RPSC electronics drawer is made through a cable described in Figure 3.3, which plugs into ELCO receptacle J103 at the Transparency, and J209 at the RPSC drawer.

e. The connection to the DDES-dedicated $120 \mathrm{VAC} 1 \emptyset$ line is made through a 3 -wire cable terminating in a standard 3-prong, parallel blade plug. The green wire is grounded to the Transparency chassis at the rear, while the other leads go to the $+12 \mathrm{~V}$ DC supply, from which jumpers carry it to the other supplies and the Bridging Relay circuitry. The plug goes to a socket in the chassis in the center of the Power Drawer. This provides a protective ground to the Power Drawer. Power comes through an isolation transformer at the left rear of the drawer. This is fed from the DDES AC line via a black twistlock 3-prong plug.

\subsubsection{Power Supplies}

a. $+12 \mathrm{~V} \mathrm{DC}--$ This is a Power-mate Corp. Model ICX I2AC regulated supply, required for the RS-232B signal levels, and is located in the left rear of the chassis. Its operation is shown by a RED indicator lamp on the front of the drawer.

b. $12 \mathrm{~V}$ DC--This is another PMC ICX 12AC regulated supply, required for the RS-232B signal levels. Its operation is indicated by a BLUE indicator lamp on the front of the drawer.

c. +5V DC--This is a PMC ICX 5A regulated supply required 
$\overline{\overline{0}}$
$\frac{0}{3}$
$z$
$\frac{1}{\pi}$
0
$u$
0
$\frac{1}{\pi}$
$\frac{\pi}{0}$
0
$\frac{0}{0}$
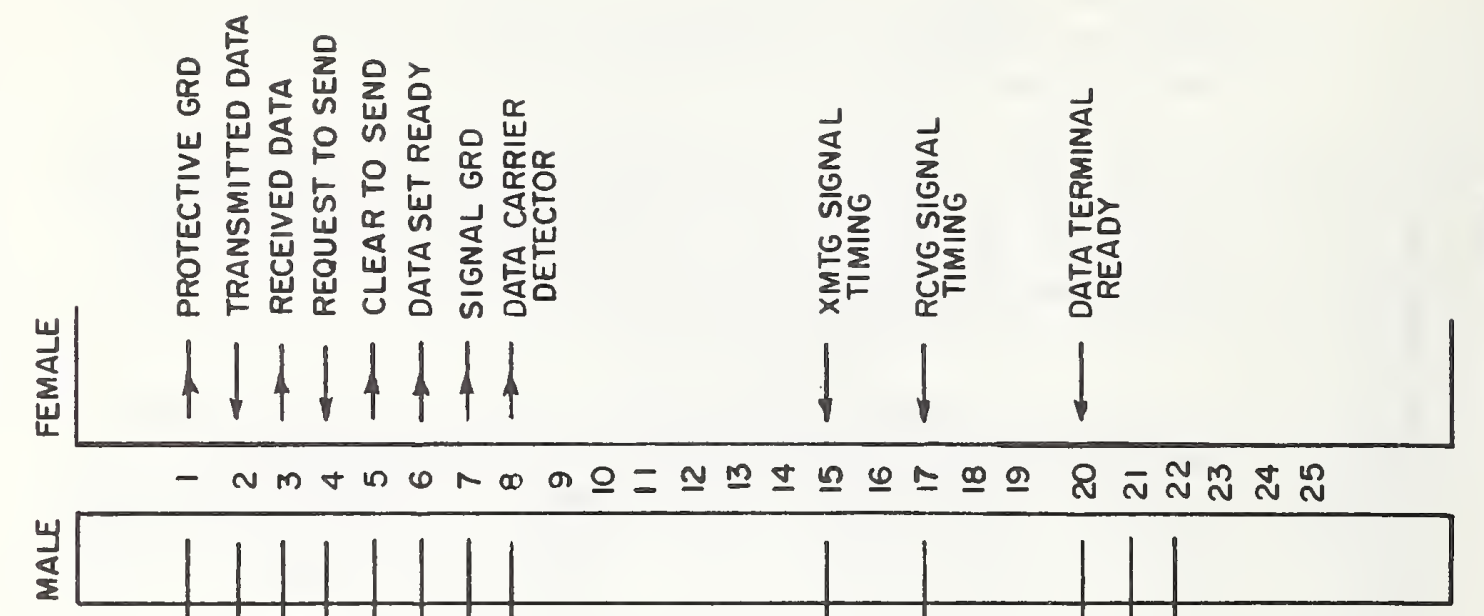

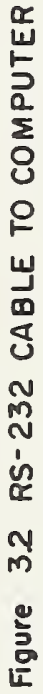

$\frac{1}{2}$
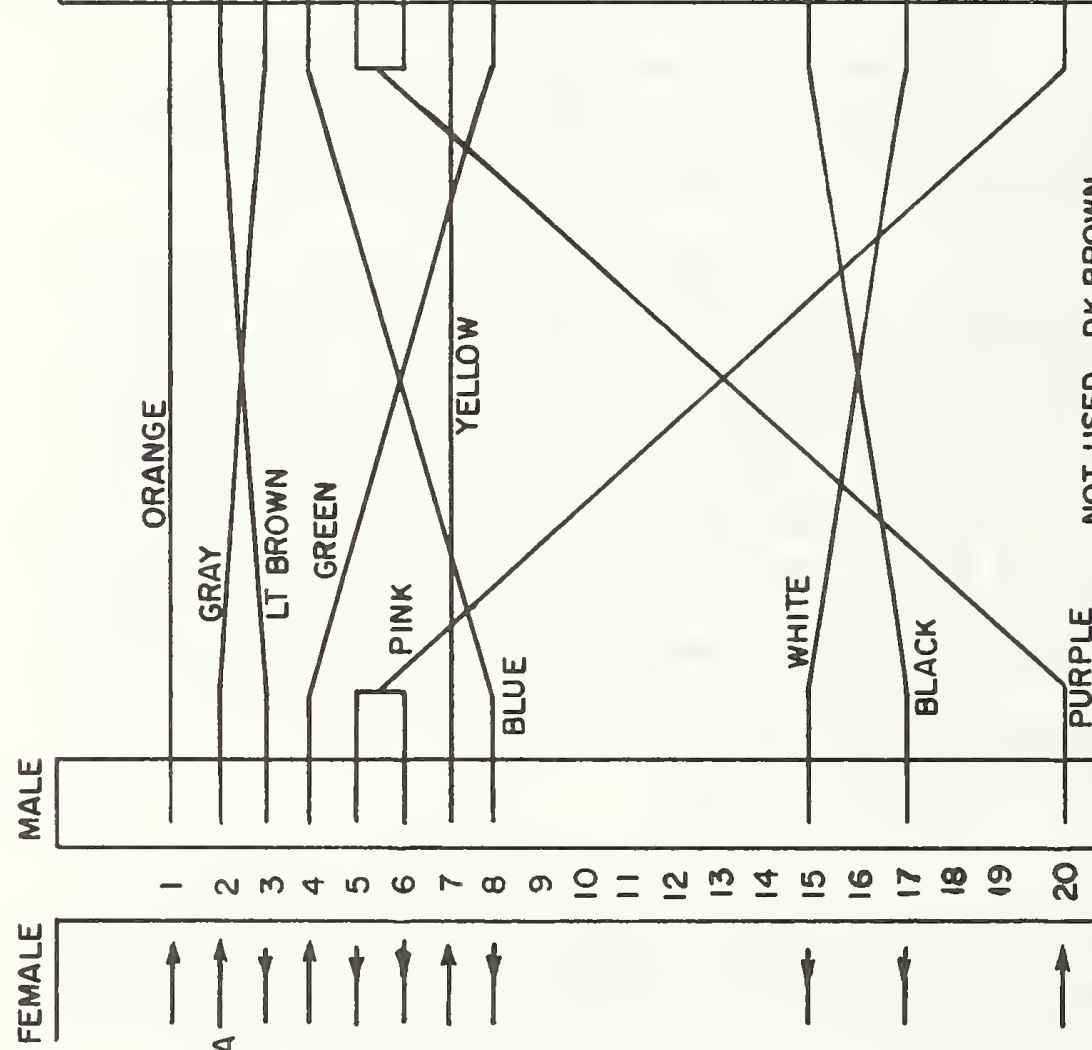

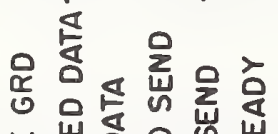

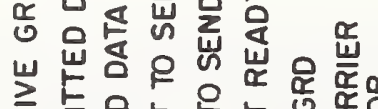

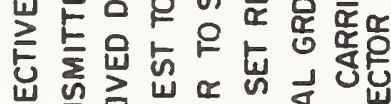

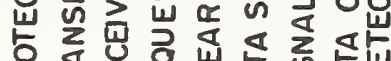

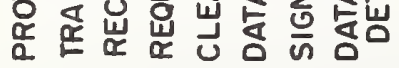

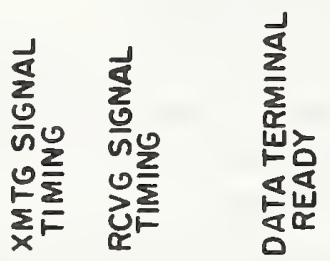




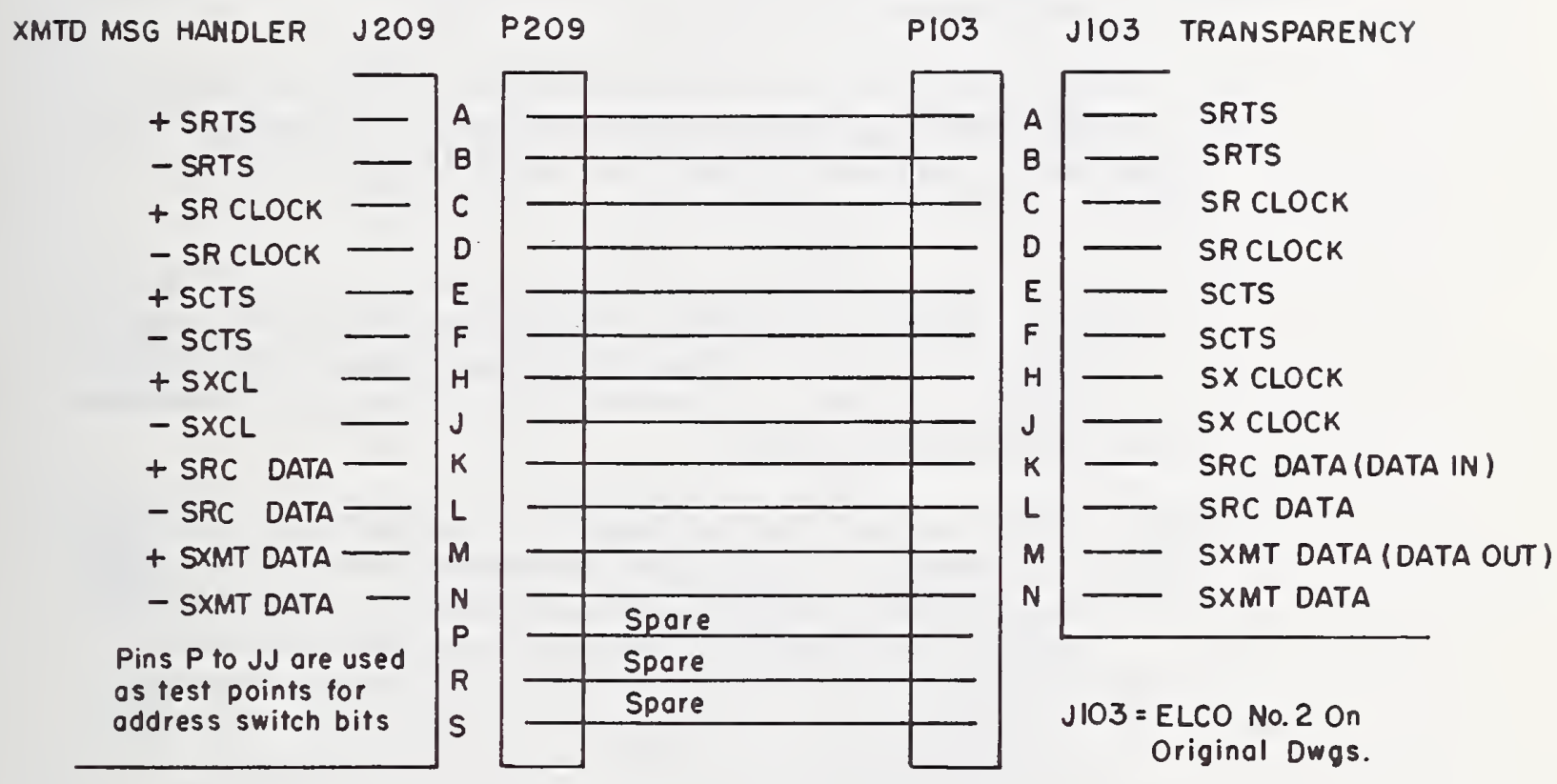

J9 On Original Dwgs.

Figure 3.3 INTER-DRAWER CABLE RPSC 
for the Transparency logic electronics. Its operation is indicated by a YELLOW indicator lamp on the front of the drawer.

d. Bridging Relay--This is a T-BAR Relay with a 120 VAC coil. Current to it is controlled by an electric relay which is driven by the DC power supply voltages. Should any voltage fail, the relay will deactivate, causing the Transparency to be bridged across J101 and J102, thus preventing the RPS from interfering with DDES operations. See Drawing No. 2.1.10.

\subsection{Electronics}

3.3.1 Transparency Functions--The Transparency provides two functions :

a. It supplies a tap on the CC-DCU Iine such that the Received Message Handler can recelve all messages sent by the CC without interfering with normal message reception by the DCU;

b. It provides a priority system to prevent interference between messages from the DCU to the CC and those from the RPS to the CC. These functions must be carried out with electrical isolation being maintained between the DDES and the RPS.

3.3.2 Logic Wiring--The logic wiring is shown on drawings 2.1 through 2.1.9. The pin numbers shown are chip pin numbers, which do not necessarily coincide with socket pin numbers. Chips are usually mounted so that the first row of socket pins is left empty. The chip location is given by the letter and number placed next to the logic symbol, and represent the coordinates shown in the Transparency chip layout, Figure 3.4. The RS-232B Line Drivers are marked ET, the Line Receivers are marked ER. These are the only chips requiring the $12 \mathrm{~V}$ DC supplies. All others require only $+5 \mathrm{~V}$ DC. 


\begin{tabular}{|c|c|c|c|}
\hline$K$ & $M$ & $N$ & $P$ \\
\hline DOUBLE & $\begin{array}{c}\text { TI } 215 \\
1 / 2\end{array}$ & BLANK & $\begin{array}{c}\text { TI } 148 \\
1 / 2\end{array}$ \\
\hline \begin{tabular}{l|r} 
& $R$ HDR \\
26 & 8 RES
\end{tabular} & $\begin{array}{l}\text { R HDR } \\
7 \text { RES }\end{array}$ & $8 T 15$ & $8 T 15$ \\
\hline $8 T 16$ & SN 7413 & $8 T 15$ & $8 T 15$ \\
\hline $8 T 16$ & $8 T 16$ & $8 T 16$ & $\begin{array}{c}\mathrm{R} \text { HDR } \\
7 \text { RES }\end{array}$ \\
\hline $\begin{array}{l}\text { R HDR } \\
7 \text { RES }\end{array}$ & 7404 & 7406 & 7404 \\
\hline 7400 & 7402 & 7474 & 7400 \\
\hline 9601 & DOUBLE & 9601 & $\begin{array}{l}\text { R HDR } \\
7 \text { RES }\end{array}$ \\
\hline BLANK & 8 CAPS & $\begin{array}{l}\text { DOUBLE } \\
\text { HEADER }\end{array}$ & 7404 \\
\hline 7407 & BLANK & $\begin{array}{l}2 \text { DIODES } \\
2 \text { RES }\end{array}$ & BLANK \\
\hline
\end{tabular}

Figure 3.4 INTEGRATED CIRCUIT LAYOUT-RPSC TRANSPARENCY 


\subsection{Physical}

The electronics for the Received Message Handler, together with those for the Transmitted Message Handler and most of the RPD Interface are located in the second drawer of the RPSC (Figure 4.1). The power supply $(\$ 5 \mathrm{~V} \mathrm{DC})$ for all of these is located in the bottom drawer of the RPSC. Connections to other chassis are through 56-pin Elco receptacle J209 (to the Transparency chassis) and a pigtail cable through the back of the electronics drawer to the +5V DC supply in the Power Drawer. The hardware for the logic chips is similar to that of the Transparency previously described. Chip layout is shown on drawing 2.6.1.

Connectors J201 to J208 are 56-pin ELCO connectors for cables to the RPDs, and are assigned to Channels 0 to 7, respectively. Circuitry for channels 8 through 11 are located in a third drawer known as the RPSC-ANNEX. (Figure 4.2) Signal connections are made through a single cable from the ANNEX drawer to the RPSC drawer. Power connections are routed from the RPSC Drawer to a barrier strip on the back panel of the ANNEX drawer. Connectors J209 to J211 are 56-pin E1co connectors for cables to the RPD's and are assigned to channels 9 to 11, respectively. (See Figure 4.5 RPD to RPSC Cable).

The address switches for each channel are two octal thumb switches on the front of the drawer. The channel number is shown on LED displays above each pair of switches.

\subsection{Electrical}

\subsubsection{Power Supplies}

Power for all circuitry except that supplied from the DDESdedicated supply comes from the RPS-dedicated 120 VAC $1 \emptyset$ line. A 20-ampere three-prong twistlock plug is used to connect into this line. This plug is of white plastic to distinguish it from the DDES-line plug. Its cord leads to an isolation transformer in the left front of the Power Supply Drawer. This transformer is tapped so that a proper mean value of output voltage may be obtained. The transformer feeds a Power-Mate ICX 5B power supply, which provides all the regulated $+5 \mathrm{~V}$ DC needs of the RPSC except for those of the Transparency.

\subsubsection{Plugs and Cables}

As mentioned above, $+5 \mathrm{~V}$ DC power comes through a cable from 


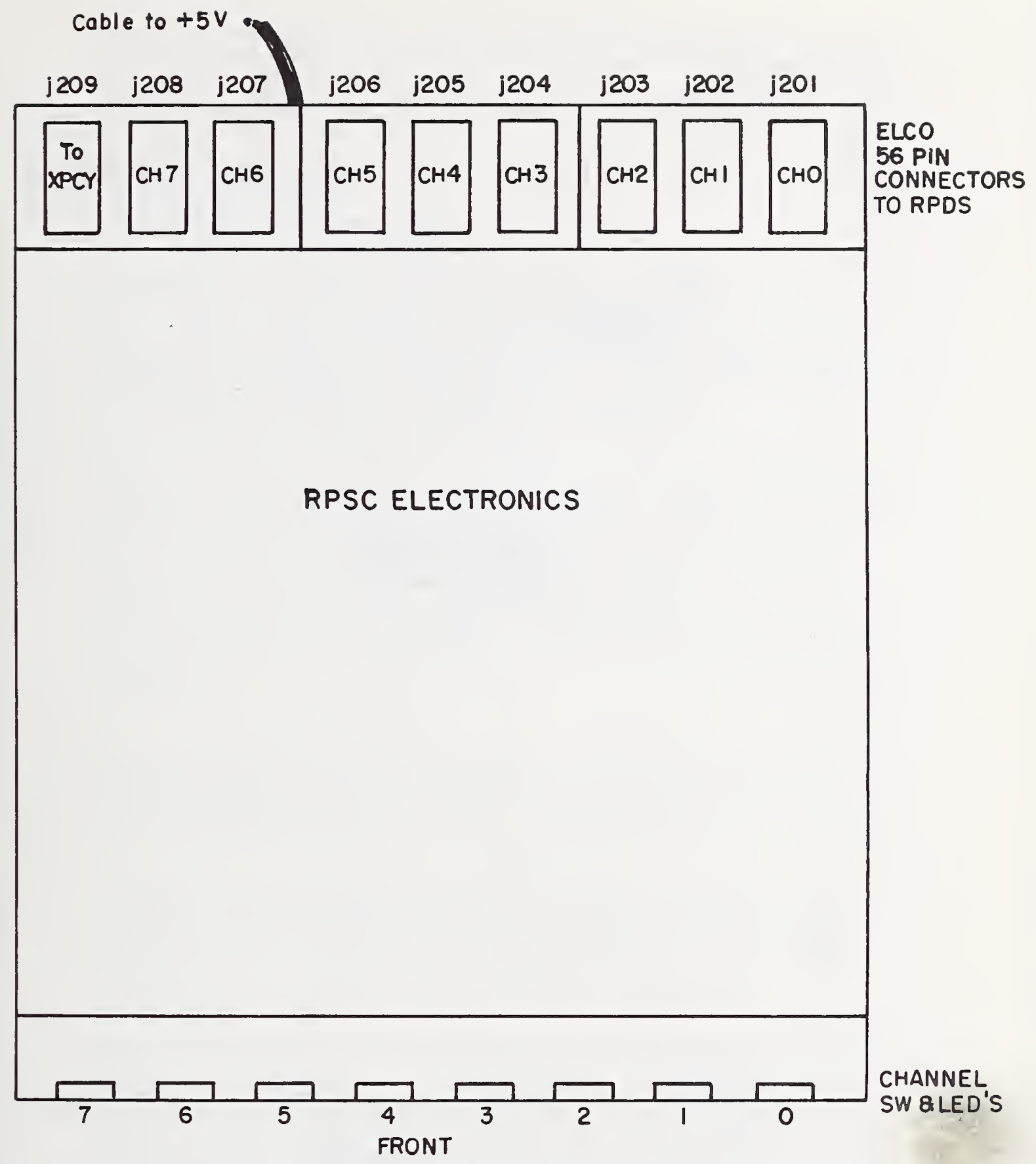

Figure 4.I MAIN RPSC ELECTRONICS DRAWER - TOP VIEW 


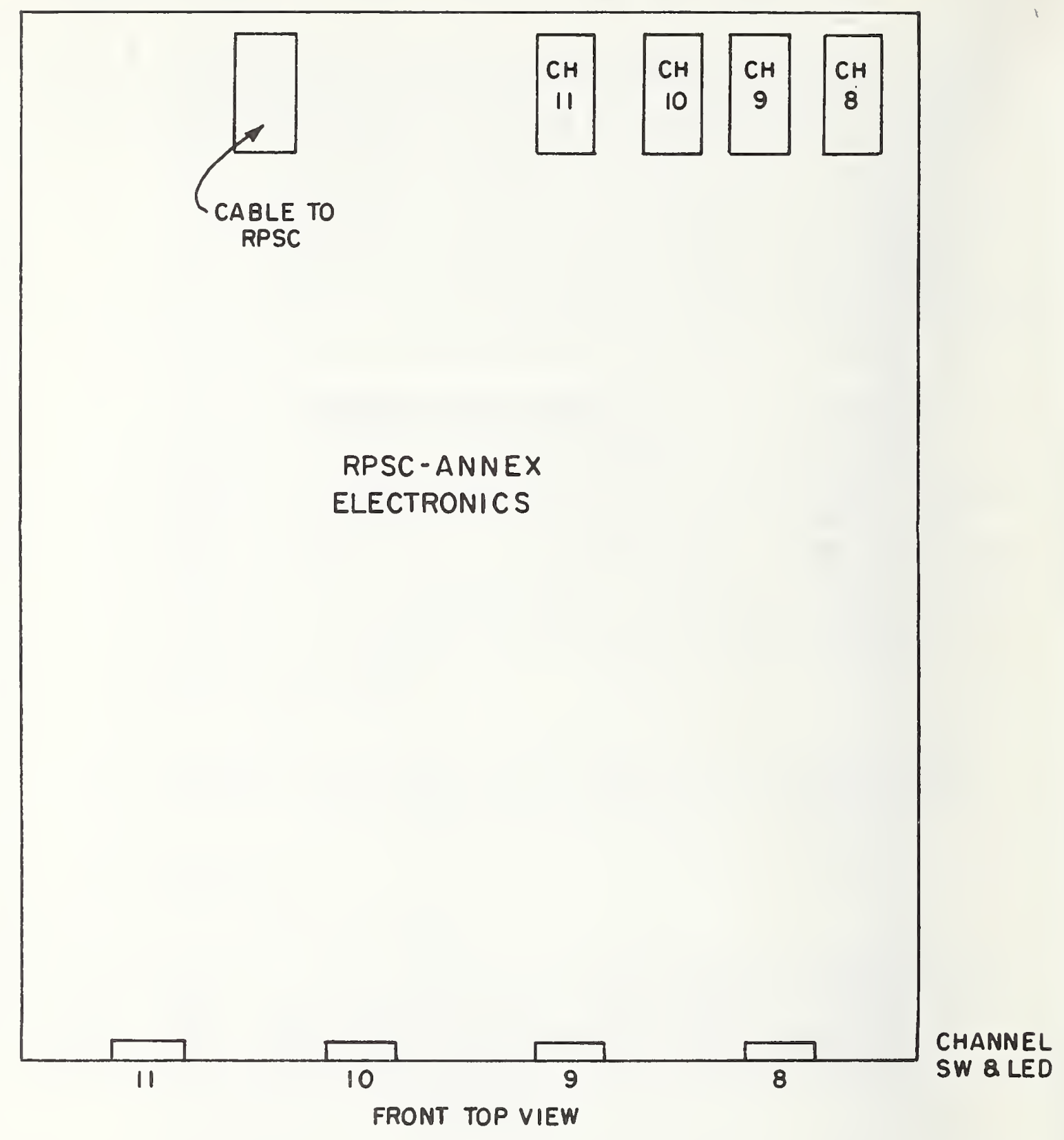

Figure 4.2 RPSC-ANNEX ELECTRONICS DRAWER 
the Power Drawer to the rear of the RPSC Electronics

Drawer. The connection to the Transparency Bridging Relay is shown in Drawings Nos. 2.1.1, 2.1.5. Connections to other parts of the RPSC are through socket to socket wiring.

\subsection{Message Processing}

\subsubsection{Message Header Checks}

All messages coming from the CC have the format shown in Figure 4.3. The message text formats are shown in Figure 4.4. They are sent synchronously as seven-bit ASCII characters with odd parity in Bit 8 , at 3520 baud/second. The header checks are made over the duration of the header in a series of operational phases as follows:

Phase 0--This is the phase in which the Received Message Handler is set by Master Reset (MRSET) at power turn-on and to which the logic is returned whenever a message has been found faulty or has been incompletely received. In this phase, the incoming message is examined in the Buffer Register at each bit time to determine whether the character in the buffer is a SYN $\left(026_{8}\right)$. When such a character is found, the Character Clock is reset and phase is advanced to Phase 1.

Phase 1--One character time ( 8 bit times) after the first SYN was found, the Buffer Register is again examined to determine whether it contains a SYN character. If not, phase reverts to Phase 0 . If so, phase advances to Phase 2.

Phase 2--In this phase, the Buffer Register contents are examined every Character Time. If the character in the register is SYN, the phase is continued; if it is an $\mathrm{SOH}$ $\left(\mathrm{OOl}_{8}\right)$, phase is advanced to Phase 3 ; if it is any other character, the Phase Counter is reset to Phase 0 .

Phase 3--The character in the Buffer Register one character time after the start of Phase 3 is taken to be the address character. If the two major bits of the address (not counting parity) are not 10 , this indicates this is not an RPS address, and phase reverts to Phase 0 . If they are 10, the address is transferred to the Address Register. If the address does not match one of the Channel Address Switch settings, the phase reverts to Phase 0 . If a match is obtained, the phase advances to Phase 4 .

Phase 4--The characters following the address character are examined during this phase. An SOH or ETX will cause a NAK message to be initiated and an abort signal to be sent to the RPD Interface. Phase will revert to Phase 0 . Should an STX (0028) be found, header processing is completed and 

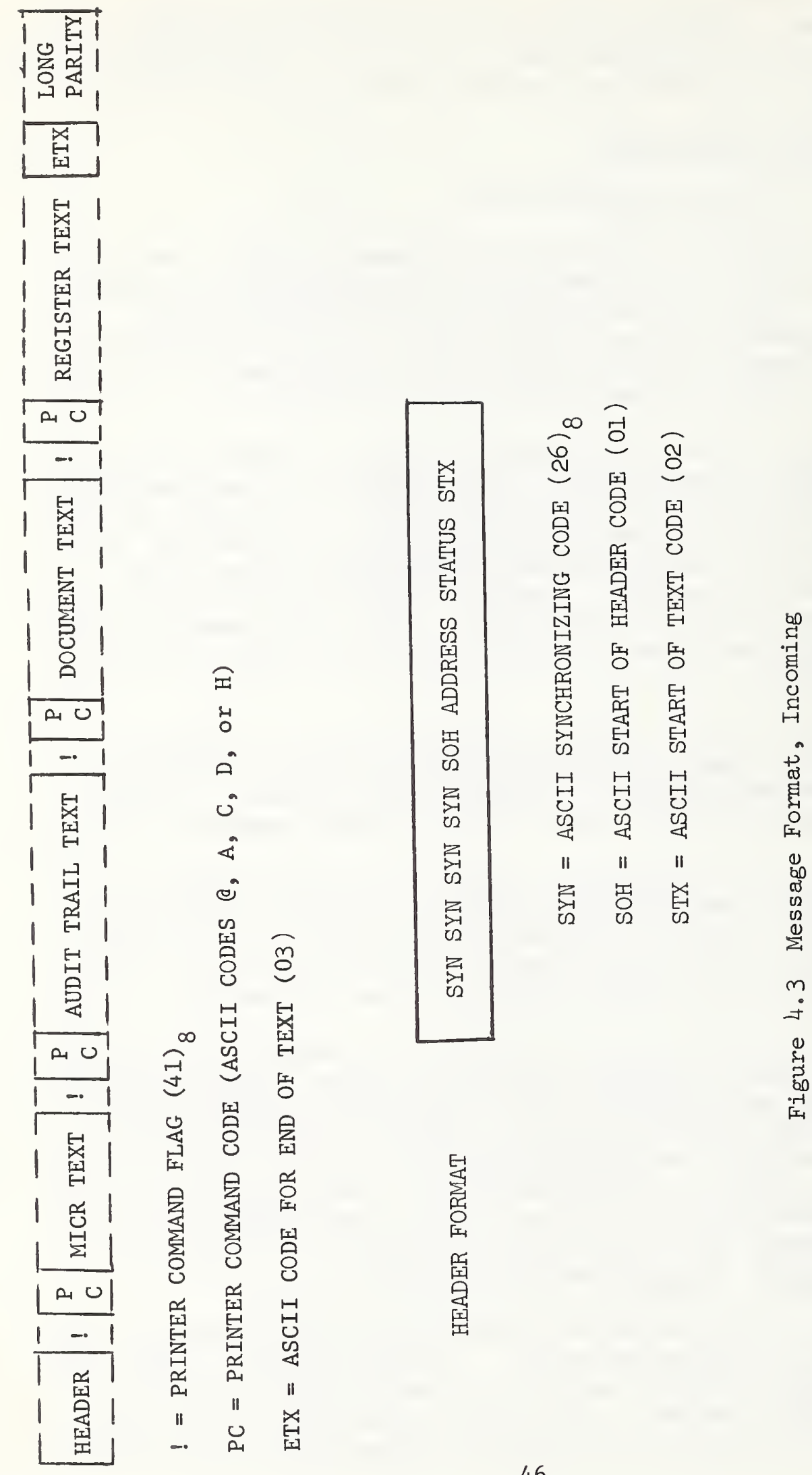

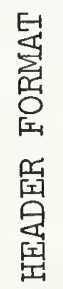


the phase advances to Phase 5. Any other character has no effect, and Phase 4 continues.

\subsubsection{Message Text Processing}

This consists of checking the format of the text, which must be shown in Figure 4.4 providing longitudinal parity, removing no-text message characters from the stream going to the RPD Interface. These checks are carried on in phases as follows:

Phase 5--The characters following the STX are examined. The occurrence of SYN, SOH, or STX within the message will cause an abort and return to Phase 0 . The occurrence of ETX $\left(003_{8}\right)$ indicates the end of the message text. If exactly four Command Flags have not been received, a NAK message will be initiated, an abort will occur, and phase will revert to Phase 0 , otherwise it will advance to Phase 6.

Every time a Command Flag is found $\left(!, 041_{8}\right)$ it is counted and a signal is sent to the RPD Interface to cause it to send a Receive Command order to the RPD. The next character is to be taken as a printer command. The RPD determines whether it is a legal command, and if it is not, will cause an abort.

\subsubsection{Trailer Processing}

During Phase 6, the ETX is sent as the last character of the printer message. The DEL sent by the CC is not sent to the RPD, but the RPD Interface will send a Receive LP command to the RPD, and the longitudinal parity character will be sent to the RPD instead. Phase then advances to Phase 7.

Phase 7--Time is allowed for the RPD to compare the longitudinal parity character with the one it has generated from the incoming message. If the two agree, and there is no other reason for the NAK message to be sent, an ACK message will be initiated by the RPSC. If a signal that a NAK is to be sent has been set, and phase has advanced to this point, the message is aborted, and a NAK is initiated. Phase then reverts to Phase 0 to await the arrival of the next message.

\subsection{The RPD INTERFACE}

4.4.1 The RPD Interface is the link between other sections of the RPSC and the RPDs. It performs the following funct1ons:

a. Transmits commands and data to the RPDs; 
1 Command Flag

1 Print Command

or

0 to 10 Decimal Digits

1 Breaker

$$
\begin{aligned}
& 041_{8} \\
& 100_{8} \\
& 100_{8}
\end{aligned}
$$

$053_{8}$
Meaning DO NOT PRINT

Meaning PRINT AMOUNT FIELD

Remittance Amount

Meaning END OF MICR MESSAGE

THE CHECK MESSAGE

1 Command Flag

1 Print Command

or

1 Pointer Symbol

9 Numerics

1 Space Symbol

4 Numerics

'I Space Symbol

1 Pointer Symbol

3 Numerics

1 Space Symbol

10 Numerics

1 Pointer Symbol
!

@

B

;

?

?

;

?$$
\text { ; }
$$

THE DOCUMENT MESSAGE

1 Command Flag

1 Print Command

or

14 Numerics

1 Space

9 Numerics

1 Space

10 Numerics

1 Breaker
!

@

D

$\not b$

$\not 6$

$+$
DLN

TIN to 10)

\author{
Meaning DO NOT PRINT \\ Meaning PRINT AUDIT TRAIL \\ Printed shape to be defined \\ TIN \\ Note this is not ASCII Space \\ $\mathrm{T} / \mathrm{P}$
}

$\mathrm{T} / \mathrm{C}$

DLN

THE REGISTER MESSAGE

Meaning DO NOT PRINT

Meaning PRINT LABEL MESSAGE

ASCII Space

Amount (may be made variable 0

Meaning END OF AMOUNT

1 Command Flag

1 Print Command

or

A variable number of al

1 End of Text Code ETX

!
(2) Meaning DO NOT PRINT

H Meaning PRINT REGISTER MESSAGE

Figure 4.4 Message Text Formats 
b. Receives status signals from the RPDs;

c. Reports status to the RMH;

d. Provides for address selection for each RPD channel;

e. Provides storage for the address selection for the TMH;

f. Initiates transmitted messages at the request of RPDs;

4.4.2 Communications with each RPD--A cable to each RPD contains circuits for the following functions:

a. Transmit printer messages from the RPSC to the RPD;

b. Transmit commands from the RPSC to the RPD;

c. Transmit status from the RPD to the RPSC;

Transmission between RPSC and RPDs is in star fashion. Disconnection of any RPD, or turning off its power will not affect other RPSC-RPD communications. RPD and RPSC interconnections are shown on Figure 4.5 with connector pin designations and signal names.

4.4.3 Signals from the RPSC--The RPSC communicates the following to the RPDs:

a. A reset or abort command to cause the RPD to go to the Idle state;

b. A selection command to prepare the RPD to receive message data and data control commands;

c. That the next character will be a data control command;

d. That the next character will be a longitudinal parity character.

It also transmits those characters of the computer message to permit proper printing of the required messages.

4.4.4 Status Messages to the RPSC-- The RPD Interface w111 receive from the RPDs indications of their being in one of the following states:

a. Inoperative (no DTR)

b. Idle (DTR) 

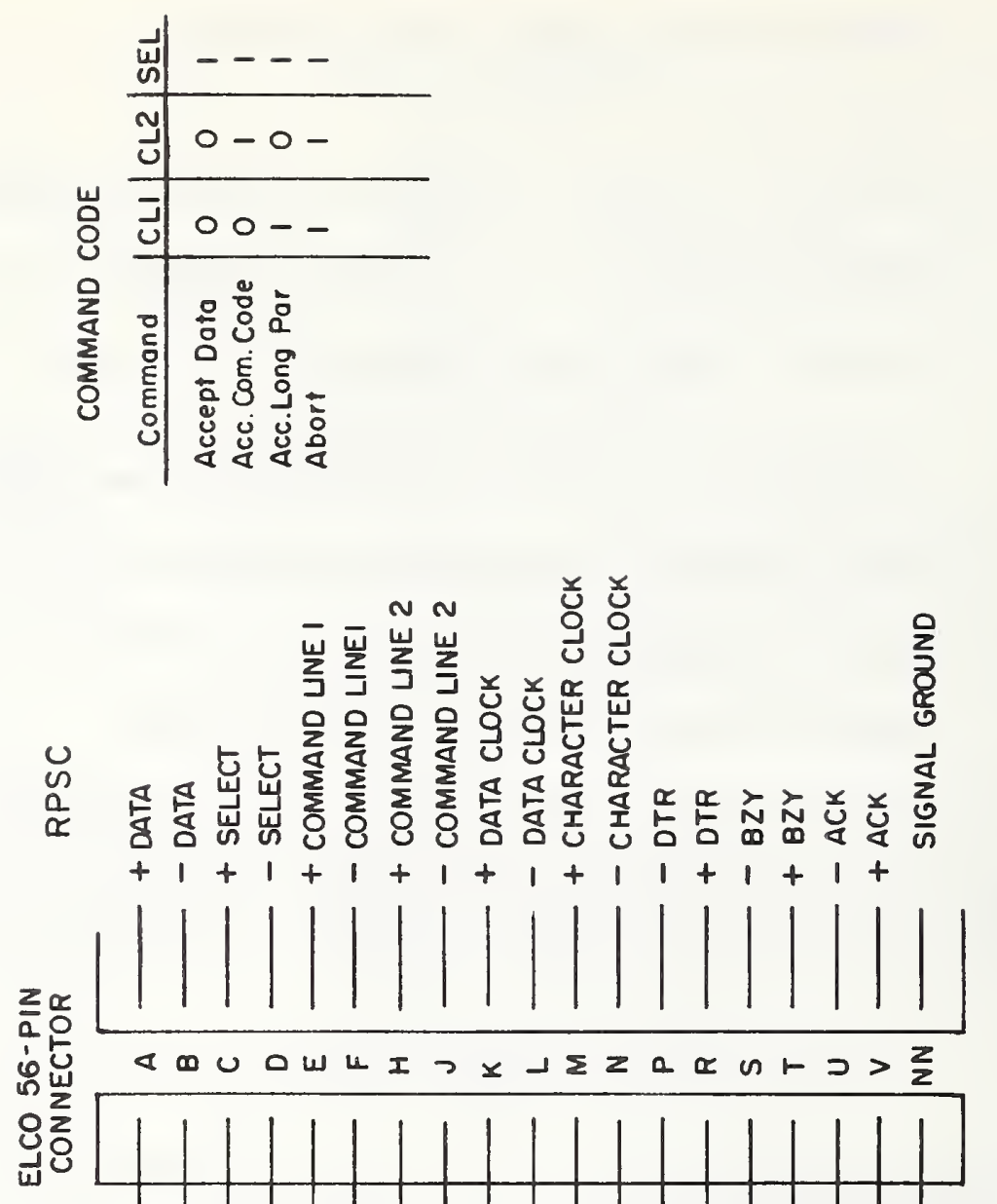

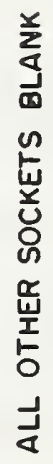

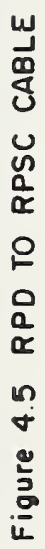

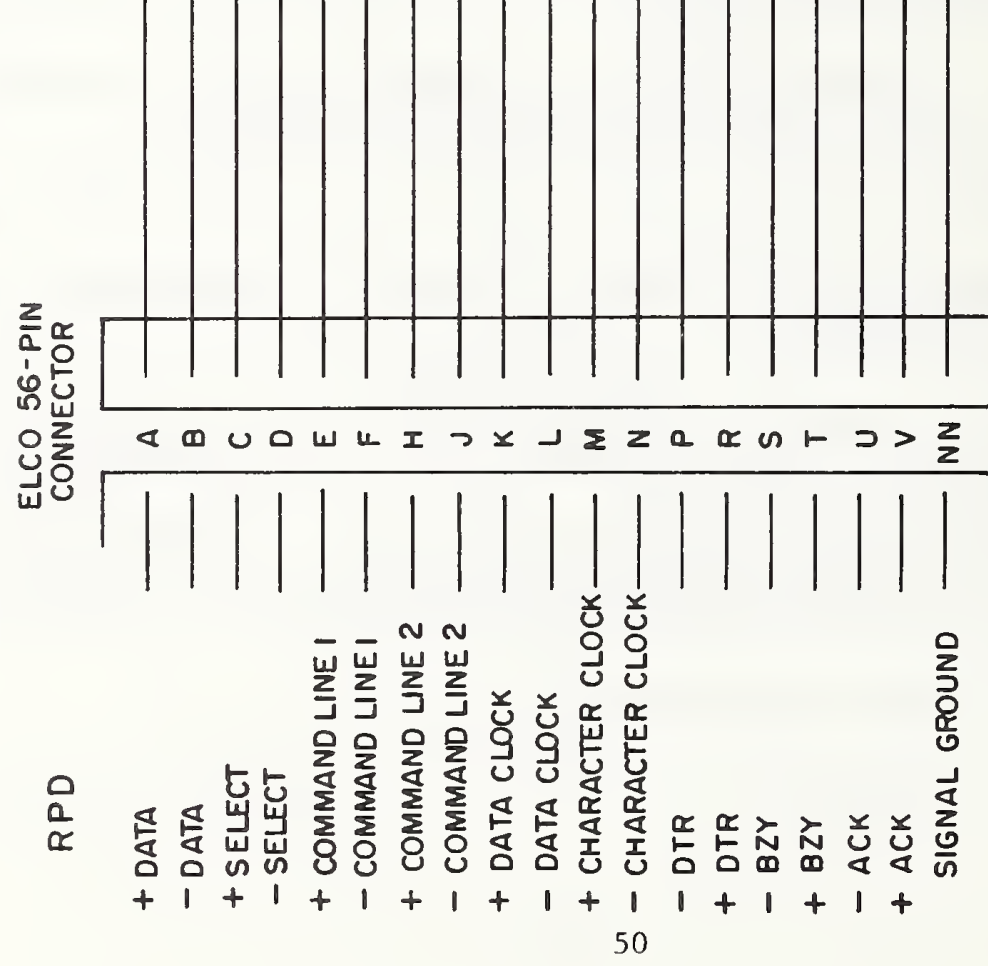


c. Busy

d. Aborting the message

The RPD Interface will recognize these signals and cause the proper RPSC actions to occur. For example, a change from Busy to Idle some time after message reception requires an ACK message to be initiated. However, as far as the RMH is concerned, the RPD will still be busy until the message has actually been sent.

\subsubsection{Address Selection}

Any RPD may be assigned to any five-bit address by means of two octal code switches obove each RPD channel cable termination. The five-bit code representing the address set into the switches is available to the TMH for inclusion in transmitted messages. They are also available to the RMH for use in making address validity checks. The octal equivalent of the code which is set is easily read by the person setting the switches.

\subsection{THE RPSC CONTROL CIRCUITRY}

\subsection{Physical}

The RPSC Control circuitry shares space in the second drawer of the RPSC and its physical arrangement has been described in paragraph 4 of this section. Drawing No. 2.6.1 lists the IC chip locations referenced on the drawings. The logic circuit diagrams are shown on drawings Nos. 2.3.1 through 2.3.7. The timing diagrams for the clock signals and their relationship to data control signals are shown on figures $5.1,5.2$, and 5.3 .

The control signals to and from the computer, DCU and RPD via the transparency are shown on Figure 3.5 .

\subsection{The RPSC Control Panel}

5.2.1 The RPSC Control Panel provides control switches and indicators necessary for the operation of the RPSC, and for power and signal connections for test purposes.

5.2.2 Control Switches--The Control Panel provides switches for the following functions:
a. Power
On/Off
b. Operating Mode
Normal/Test 


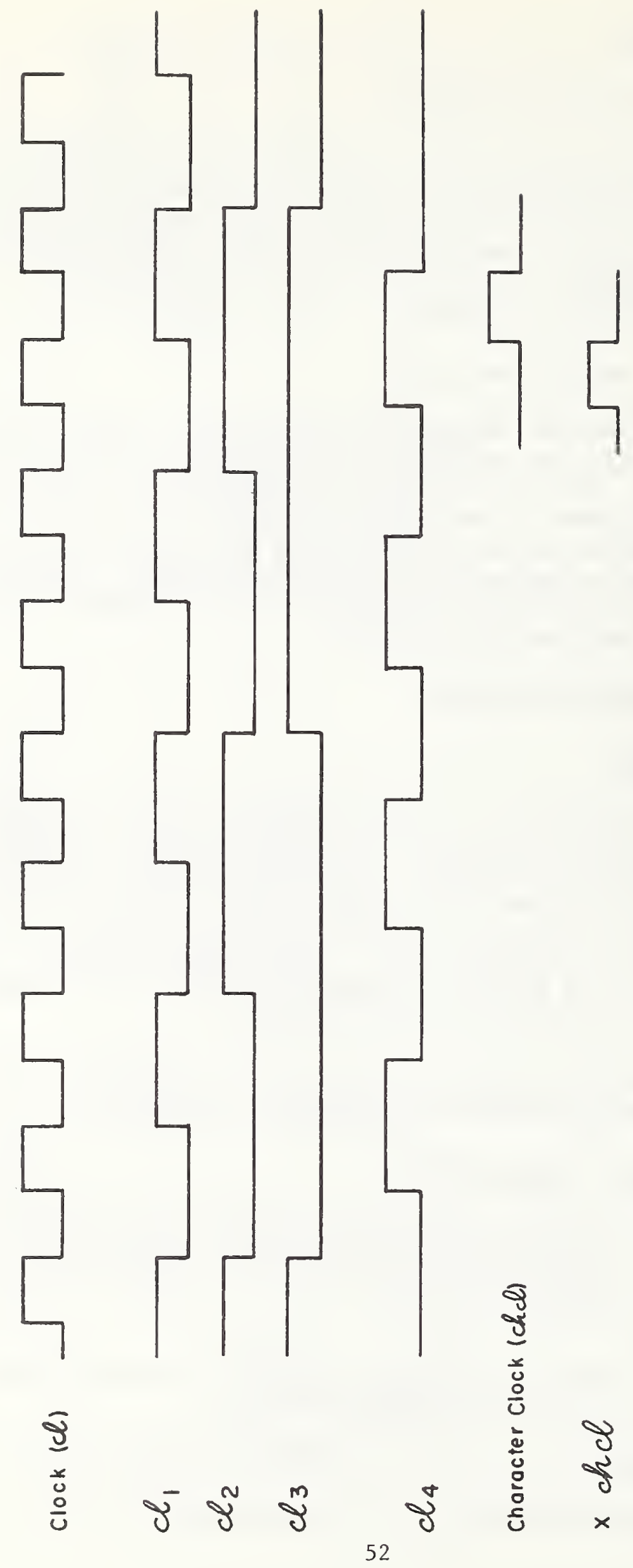

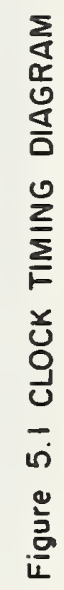



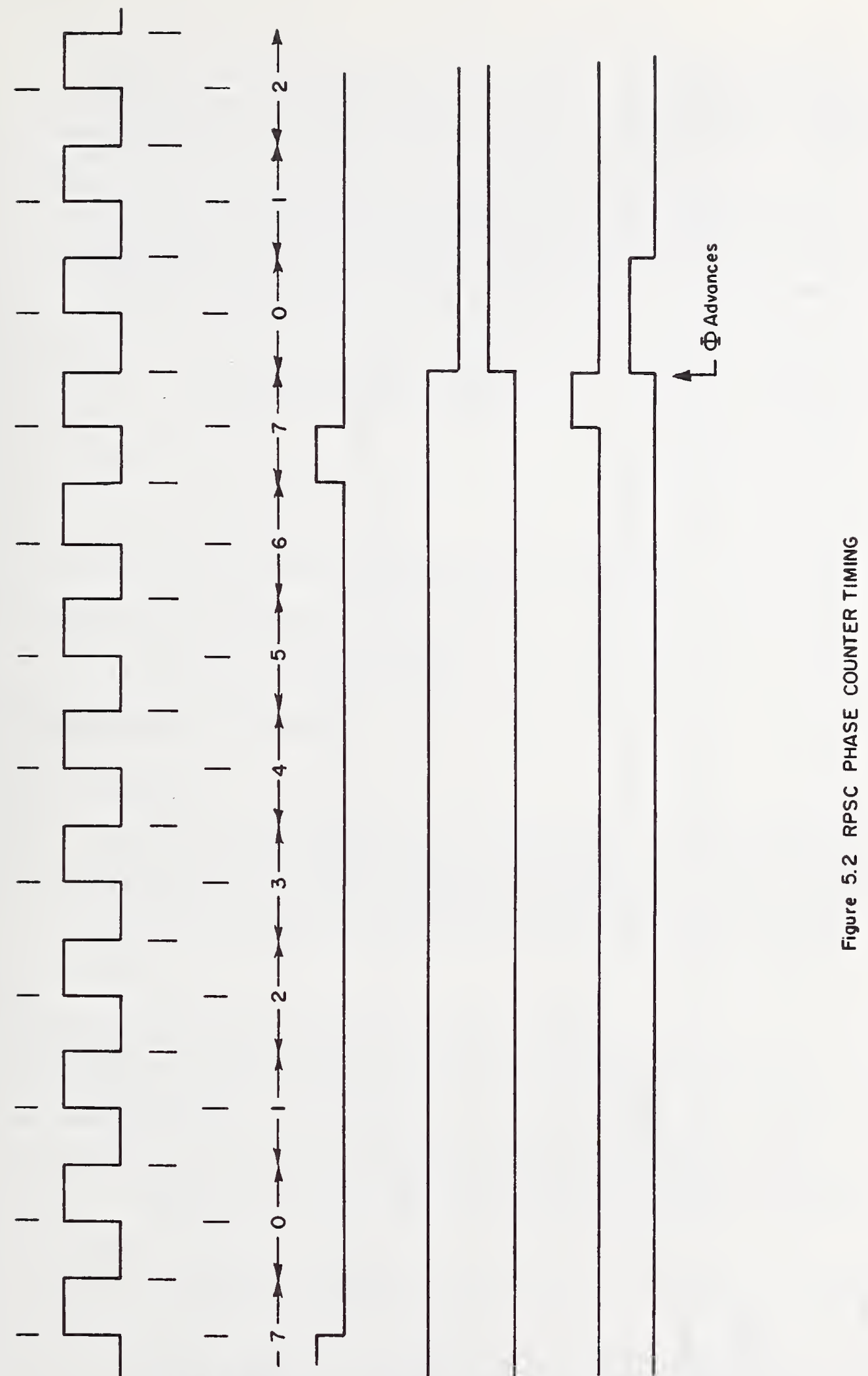

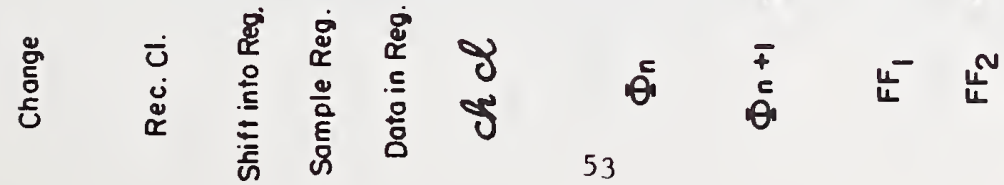




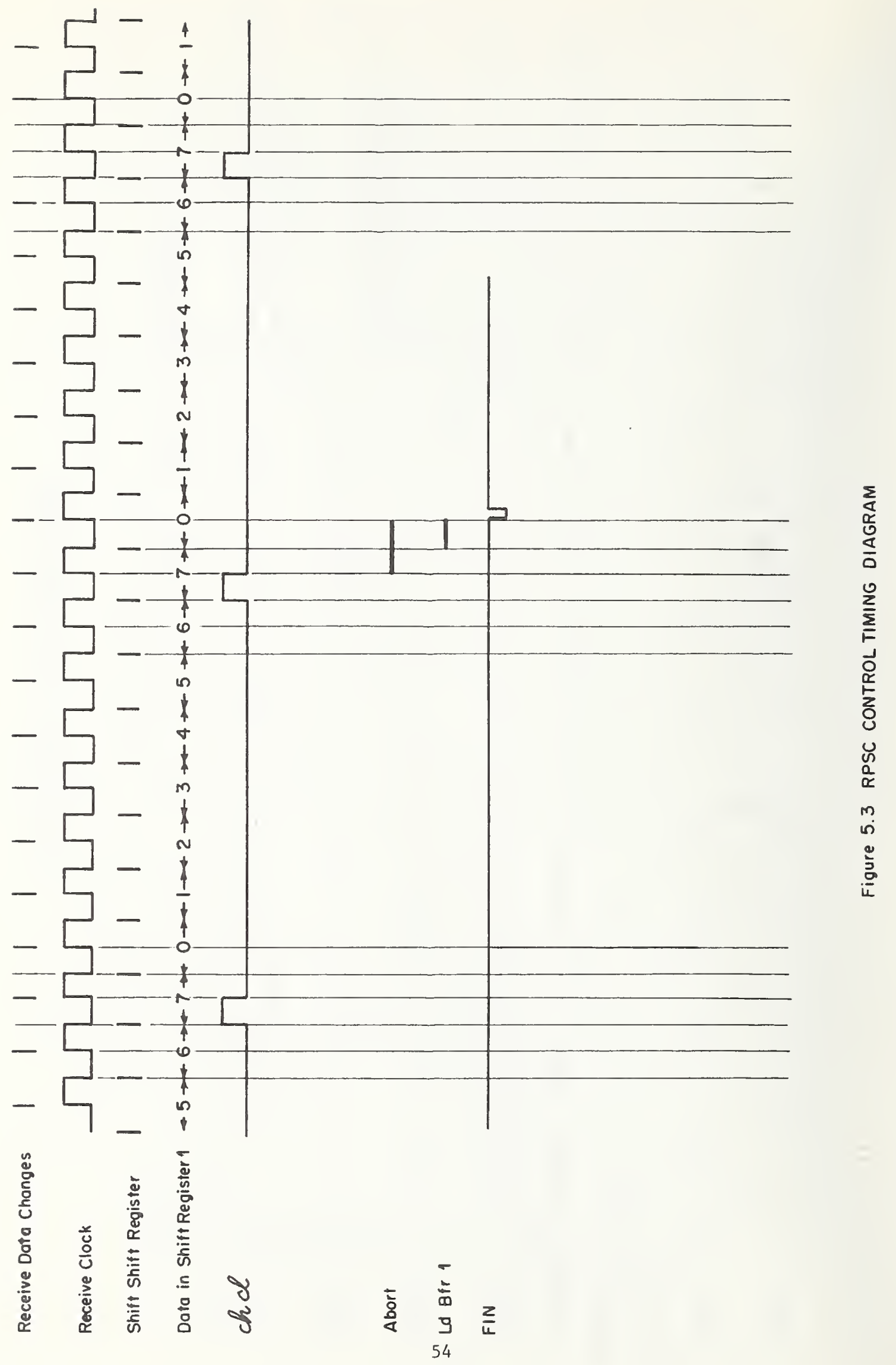


The Mode switch operates the Interface bridging relay, so that in the Normal mode the RPS-DDES Interface is connected between the CC and the DCU, while in the Test mode, the CC and DCU are bridged together around the RPSC.

The Clock Source switch connects the RPSC clock into the circuit in the Internal Mode only if the DCU clock is inoperative, or the bridging relay contacts are closed. In the External mode, a clock input will be accepted from either the CC, DCU, or Test connectors.

5.2.3 Indicator Lamps--The RPSC Control Panel contains the following signal lights:

a. Power On

b. AC Power Fuse Failure for each power supply

c. DC Power supply failure for each supply

d. Test Mode Selected

e. Internal Clock Selected

\subsection{THE RPSC REPLY CIRCUITRY}

\subsection{Physical}

The RPSC Reply Circuitry is located in the second drawer of the RPSC and its physical arrangement has been described in paragraph 4 of this section. Drawing No. 2.6.1 lists the chip locations shown on the logic circuit diagrams. The logic circuit diagrams are shown on drawing Nos. 2.4 2.8 .

\subsection{The Transmitted Message Handler (TMH)}

The Transmitted message handler performs the following functions:

a. Assembles and transmits a response message to the computer via the RPS-DDES Interface at the initiation of the RMH or the RPD Interface;

b. Provides the priority rules when more than one message is awaiting transmission;

c. Provides for limited storage of messages or message data until response messages may be transmitted; 
d. Provides for disabling of the RMH when this storage has been filled.

\subsection{Transmitted Message Format}

The format for a transmitted message is the same as that of a received one, except there is no information between STX and ETX. The only variables are the address and the status. The address is assembled as follows:

The two major bits are 11.

The five minor bits are taken from an address register in the RMH for RMH-initiated messages, and from the code wheels for the indicated RPD in the RPD Interface, for RPD-initiated messages.

The parity bit is generated to give odd parity for the assemblage of these bits into a character.

The status code is always ACK for RPD-initiated response messages, although an RPD signal may cause an RMH-initiated NAK. It will be only ACK, NAK, or BSY for RMH-initiated messages according to signals from the RMH. The BSY code is 1578 (ASCl1 for 0 ). The physical format is the same as that of received messages.

\subsection{Message Initiation}

Messages are initiated only by signal from either the RMH or the RPD Interface according to their functions. There is no means for a request for repetition provided in the system, and any storage in the computer used for the message is released for another message as soon as the message has been sent.

\subsection{Message Priority}

An RMH-initiated message takes precedence over an RPD Interface-initiated message. RMH-initiated messages are taken in sequence; the oldest message first. RPD Interfaceinitiated messages are taken in order of channel number.

\subsection{Transmitted Message Storage}

Storage is provided for at lesst two RMH-initiated messages and for at least one message from each RPD channel.

Since only two characters are variable in the message, only these, or signals of choice of these need be stored. For example, for an RMH-initiated message, storage need consist of only two 7-bit words, with five bits being used to hold 
the address, and the other two to select the status. For RPD-Interface-initiated messages, there is only one type, and the address is stored in the switches associated with the channel, so only one bit is necessary to indicate all information necessary per channel.

\subsection{RMH Lockout}

Whenever the storage provided for messages to be transmitted is fllled, the TMH prevents the RMH from handling any further incoming messages until a CTS has been received. The lockout will not occur unless the RMH is in Phase $0,1,2,6$, or 7 , so as not to interrupt processing of a possibly valid message. Should it be possible to get a lock-out signal during Phases 3,4 , or 5, storage of the necessary information for the response to the current message could be required either in the RMH or the TMH.

\subsection{Message Transmission}

As soon as the transmission of a response message is required, the TMH will attempt to seize the data line by setting the RTS signal in the RPS-DDES Interface, and await receipt of the CTS signal before beginning transmission. Once the line is seized, the TMH will continue to send messages until all storage is empty. At this time the RTS signal will be reset, in order to free the line for the DCU.

Priority with respect to the DCU will be handled by the RPSDDES Interface, using these signals.

When an RPD-inftiated message has been sent, the RPD Interface will be signalled to release that RPD from the busy state insofar as the RMH is concerned.

\subsection{THE POWER SUPPLY DRAWER}

The physical layout of the RPSC Power Supply drawer is shown in Figure 7.1. The drawer contains two isolation transformers and one PMC - IC2-5B power supply. The front panel has an a.c. switch and indication for each of the isolation transformers. One controls primary power to the transparency and the other primary power to the RPSC.

\subsection{THE TRANSPARENCY DRAWER}

The physical layout of the RPSC Transparency drawer is shown in Figure 3.1. The drawer contains three d.c. power supplies, a subpanel containing integrated circuit chips, cable connectors nos. 8 through 15, and a control panel. 


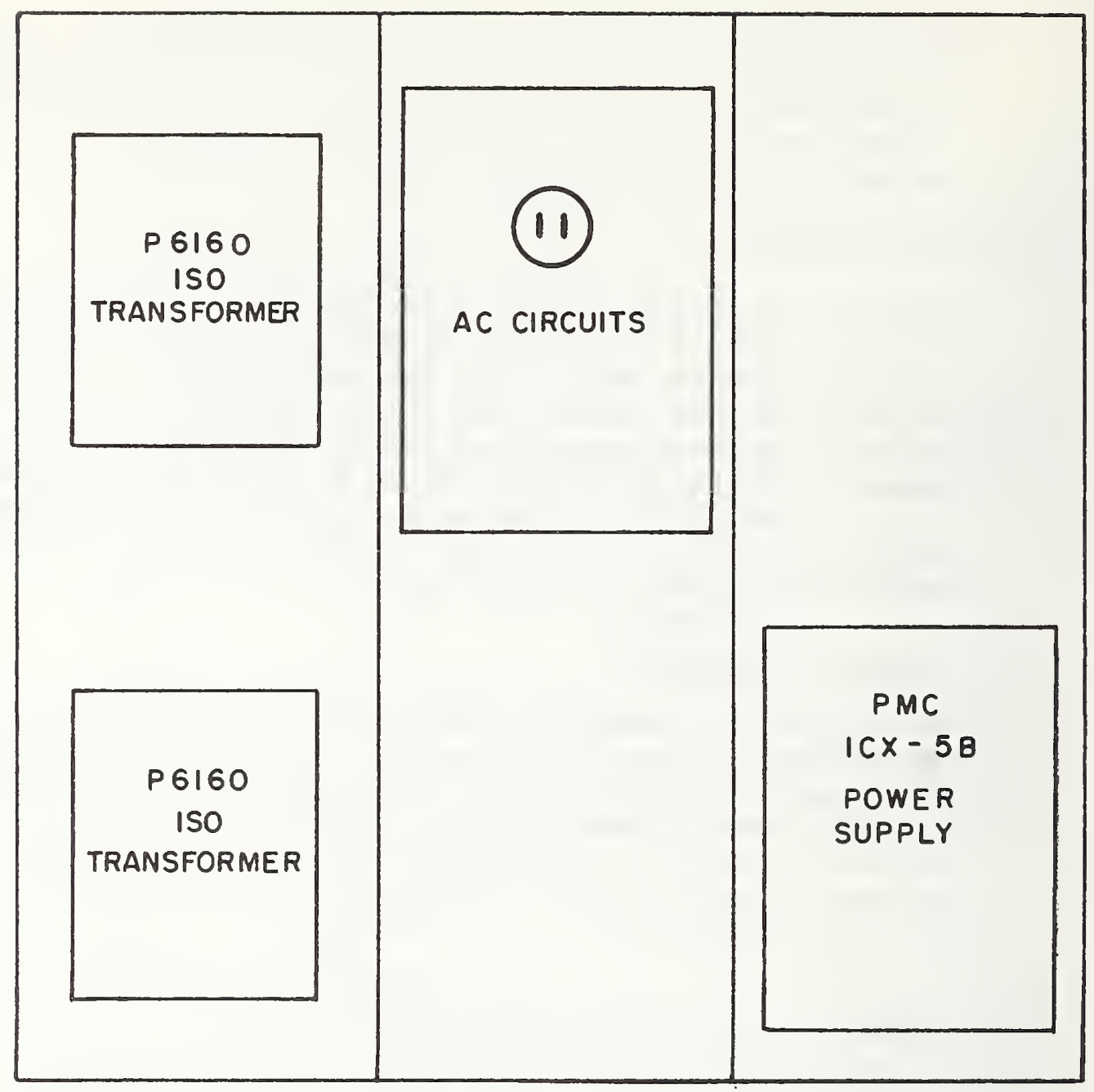

TOP VIEW

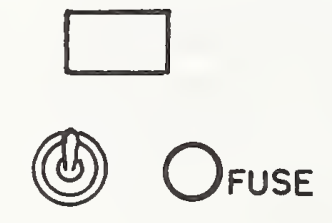

TRANS. AC
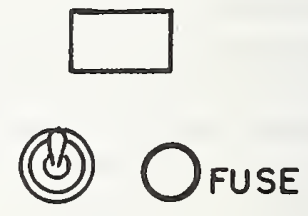

RPSC AC

FRONT PANEL

Figure 7.1 POWER SUPPLY DRAWER 


\subsection{RPSC DETAILED DESCRIPTION OF OPERATION}

\subsection{RPSC Operation}

The RPSC serves to interconnect the set of RPD's to the DDES system, via the Transparency Unit. The RPSC receives messages from the computer and routes them to the designated RPD. It recelves responses from the RPDs and formulates them into messages for the computer (ACK, NAK, or Busy). It is capable of accepting messages from the computer and transmitting to the computer simultaneously. The RPSC does not have buffer storage for messages from the computer; instead, it sets up the transmission path to the designated $\mathrm{RPD}$ and sends the messages along as they are recelved.

The RPSC performs various error checks and generates long1tudinal parity for messages transmitted to the RPDs.

The RPSC interfaces to the Transparency Unit via the following signals:

(1) Recelved Signals -
a. Received Data: Bit-serial character stream
b. Received Clock: Continuous bit pulses
c. Clear to Send Signal (CTS): Computer available to recelve message from RPSC
d. Transmitted Clock: Bit pulses produced by Display Control Unit for timing of transmissions to the computer

(2) Transmitted Signals -

a. Data Out: Bit-serial character stream

b. Request to Send Signal (RTS): RPSC desires to send message to computer

The RPSC interfaces to the set of RPDs via the following signals, for each RPD:

(1) Transmitted to each RPD:
a. Data: Bit-serial characters
b. Clock: Bit pulses
c. X Character Clock: Character pulses


d. Select line: Selection of designated RPD

e. Control Line 1:

Coded to contain control Information-

Recelve Data, Printer Command Flag, Longitudinal Parity,

f. Control Line 2: and Abort

(2) Recelved from each RPD:

a. ACK: Acknowledgement to be sent to computer (occurs upon successful completion of printing)

b. BZ: RPD Busy

c. Data Terminal Ready (DTR): RPD operable

\subsection{Sequence of Operations in RPSC}

Processing in the RPS begins when an operator keys in information via the KST (Key Station Terminal) at an RPD indicating that a document is to be processed. Operation of the KST is completely independent of the RPSC and RPD. After the verification operation has been successfully completed via the KST, the printing of the documents can proceed. At this point, the computer sends a message containing information to be printed to the designated RPD, which is routed via the RPSC. The sequence of operations takes place as follows:

(1) The computer starts sending the message via the RS-232 transmission path shared by the RPSC and DCU.

(2) The RPSC establishes bit and character synchronism with the information being transmitted

(3) The RPSC monitors for SYN characters (at least two) and then an SOH character. The character following $\mathrm{SOH}$ is interpreted as an address character. If the two high order bits are 10 , this means that the message is intended for the RPSC, and the five low order bits are decoded to determine the designated RPD. Otherwise, the message is disregarded.

(4) If the message is for an RPD, the designated RPD is checked to see if it is avallable.

(5) If the designated RPD is ready and is not busy, the Select line to that RPD is turned on. 
(6) The selected $R P D$ responds with a Busy signal and the RPSC routes the balance of the message to it.

(7) As the messages flow through, the RPSC monitors it for certain control information and sets Control Lines 1 and 2 to the RPD accordingly:

a. Initially, the control lines indicate that data is to be received.

b. Message segments for the various printers are indicated by a Printer Command Flag and Printer Select Character. The RPD is alerted when a Printer Command Flag is detected.

c. The last message character is an ETX and is followed by the longitudinal parity character. The RPD is alerted when this character is to be transmitted.

d. If the RPSC detects any errors during transmission, it generates an abort signal and sends this information to the RPD.

(8) All print messages to an RPD should have four segments, indicated by Printer Command Flags, one for each of the four printing operations (Amount, Audit Trail, Label, Register). Each segment must appear even if it contains a control character meaning that it is to be ignored. The RPSC monitors for the occurrence of four Printer Command Flags.

(9) The RPSC transmits characters to the RPD w1th lateral parity as received from the computer. The RPSC generates a longitudinal parity character and sends it as the last character in a message, following the ETX character. (Messages from the computer have a character in this position, but it is transmitted to the RPSC as all one's).

(10) The RPSC waits for a short time (two character intervals) after the routing of a message to an RPD. If the Busy signal from the RPD is still on at the end of that time, the RPSC assumes that the message has been successfully received. The RPSC then generates a short acknowledgement message (ACK) and sends it to the computer, including in it the address of the designated RPD.

(11) If the Busy signal from the RPD goes off during or at the end of a message being sent to it, the RPSC assumes that an error condition occurred at the RPD. The RPSC 
generates a NAK message and sends it to the computer, including in $1 t$ the address of the designated RPD.

(12) If an RPD is not ready to receive a message which is designated for 1t, the RPSC ignores the message. This can be expected to result in the retransmission of the message by the computer.

(13) If the designated RPD is busy during receipt of a message for it, the RPSC generates a Busy message, including in it the address of the RPD, and sends it to the computer.

(14) The RPSC has two buffer registers for storing addresses of RPDs for which acknowledgement messages are to be sent. If a message arrives from the computer while these two buffers are full, the data input to the RPSC from the computer is inhibited. This would preclude the transmission of an acknowledgement for the arriving message, and this would be expected to lead to a retransmission of the message by the computer.

(15) After an RPD has successfully completed its printing operation, it generates an ACK signal which is applied to the RPSC. The RPSC generates an acknowledgement message and sends it to the computer, including in it the address of the RPD which generated the response.

(16) If the printing operation is not completed successfully, the RPD does not generate an ACK signal. There is no provision in the RPDs for generating a NAK signal. Instead, the RPD holds up until the operator takes action to clear the problem, so that an ACK signal can eventually be produced.

\subsection{Operation of RPSC Major Components}

The major components of the RPSC are shown in the block diagram, Figure 9.3, together with the primary interconnections among the components. The blocks contain reference numbers to the detailed logic drawings. The overall operation of the major components is discussed below.

\subsection{Recelpt of a Message from the Computer}

When the computer sends a message, it goes to the Transparency Unit, which applies it to both the DCU and the RPSC. Each unit establishes synchronism with the data stream and monitors for the address character. If the two high order bits of this character are 11, the message is intended for 


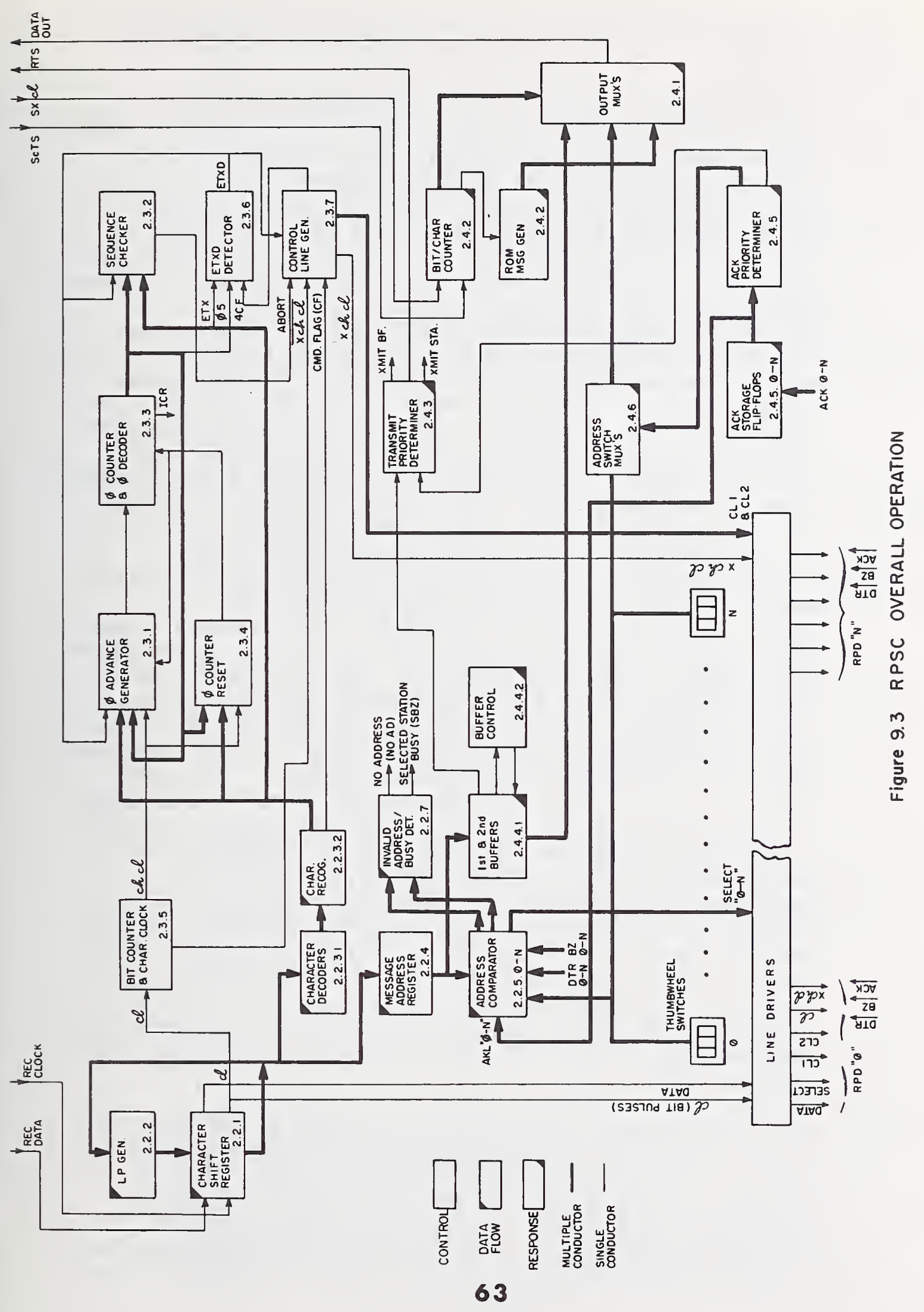


the DCU; if these bits are 10, the message is intended for the RPSC.

When a message begins to arrive, data appears on the Recelve Data line and bit pulses appear on the Receive Clock line. The bit pulses are used to shift the data signals into the Character Shift Register (Drawing 2.2.1). The bit pulses are shaped by circuitry included in this component and are applied to the Bit Counter and character Clock Generator (2.3.5). At first, the RPSC is unable to distinguish the end of one character from the beginning of the next. However, the Character Shift Register is attached to the Character Decoders (2.2.3.1) and the Character Recognizer (2.2.3.2). Whenever the pattern of bits in the shift register matches that of the SYN character (synchronizing character) the corresponding output from the Recognizer is energized. This signal goes to the Phase Advance Generator (2.3.1) which in turn drives the Phase Counter and Phase Decoder (2.3.3). It is required that all messages begin with a set of SYN characters. After the occurrence of at least two SYN characters, the circuitry will have had a chance to ascertain that the first occurrence was indeed a SYN character and not a fortuitous occurrence of the SYN pattern resulting from parts of two separate characters. The character clock generator synchronism is confirmed at this time, and the phase circuitry can begin to monitor for other characters in a specific sequence. This is done by the Sequence Checker (2.3.2), which receives inputs from the phase decoder and from the character recognizer. The phase counter is advanced by one for each character, and these must occur in the prescribed fixed message format. The next character after the initial SYN characters is the SOH (Start of Heading) character. Should a different character occur at this time, an error condition would be indicated. This would cause the Phase Counter Reset circuit (2.3.4) to generate a reset pulse which would reset the Phase Advance Generator and the Phase Counter and Phase Decoder. If the SOH is observed at the correct time, the next character is taken to be the address character. This character is transferred to the Address Register (2.2.4), where a check is made of the two high order bits. If they are 10, processing proceeds; if they are not 10, the balance of the message is ignored.

As soon as it is recognized that a message is intended for the RPSC, the five low order bits of the address character are decoded to determine which RPD the message is to be routed to. This is done by means of a set of Address Comparators $(2 \cdot 2 \cdot 5 \cdot 0-N)$. Here, the address being held in the Address Register is compared with the addresses that are set up in a set of thumbwheel switches. Each switch is set 
to a five-bit address for its associated RPD. If it is found, as a result of the comparison, that the designated RPD is ready (DTR signal present) and not busy (BZ signal off), then the Select line for this RPD is energized. This sets up a signal path to the RPD and causes the control lines to it to be activated. The remainder of the message from this point on is transmitted to the RPD. The current address is loaded into the first of two buffer registers $(2.4 .4)$ and will be used, after the message is completed, in the sending of a response message back to the computer.

During the receipt of the test of a message, the RPSC monitors for the Printer Command Flags and the ETX character. These are detected by the Character Recognizer and are used to set up signals on Control Lines 1 and 2, via the Control Line Generator (2.3.7).

During the time that a message is being routed to an RPD, the RPSC generates longitudinal parity, via the Longitudinal

Parity Generator (2.2.2). As each message character occupies the Character Shift Register it is applied to the Longitudinal Parity Generator which has a toggle flipflop for each bit position in the character. At the end of the message, the character stored in the parity generator is transferred to the shift register and sent as the last character.

Should an error occur during the transmission of a message, such as an improper character sequence in the fixed message format, an "abort" signal would be generated by the Sequence Checker. This signal is sent to the RPD via Control Lines 1 and 2 and terminates the reception of the remaining part of the message.

Generation of Response Messages--The RPSC normally generates two response messages to the computer for each message that is received. The first is an acknowledgement immediately following a message, and the second is an acknowledgement following successful completion of the resulting printing operation.

When a message begins to arrive from the computer, the RPSC checks to see if the designated RPD is ready and not busy. If the RPD is not in the ready state (DTR signal off), the RPSC ignores the message and provides no response. (This would be expected to lead to a retransmission of the message. However, the computer would signal the operator if no response was obtained after several retries.) If the RPD is busy, the RPSC generates an acknowledgement message with the status character having the code for Busy. The response message carries the address of the designated RPD in its address character. When a message is successfully transmitted to an RPD, the RPSC waits for a short time (two 
character intervals) after the transmission and then notes whether the Busy signal from the RPD is still present. If it is, the RPSC presumes that the message was successfully received by the RPD and that the printing operation is underway. The RPSC then generates an acknowledgement message to the computer with the status character containing the ACK code. The response message carries the address of the designated RPD in its address character. If the Busy signal from the RPD had gone off by the time the response message was to be generated, the RPSC would have sent a negative acknowledgement message, having the code for NAK in the status character.

The preceding response messages are generated in the following manner:

The address contained in the original message from the computer is placed in the Address Register and compared against the RPD addresses as set up in the thumbwheel switches. The address is transferred to the first of the two buffer registers for inclusion in the response message. When the time comes to generate the response message, the address is shifted from the first buffer to the second buffer. When the buffer has an address, indicating that a message is to be sent, a signal is sent to the Transmit Priority Determiner (2.4.3) which in turn raises the RTS (Request to Send) signal to the Transparency Unit. The Transparency Unit resolves any conflict between requests from the DCU and the RPSC, and sends an SCTS (Station Clear to Send) signal to the RPSC when the line to the computer is available.

The response message is generated by reading certain fixed data from a ROM (Read-Only Memory) (2.4.2) and combining with this the address for the pertinent RPD. The response messages are constituted as follows:

(1) Four SYN characters

(2) $\mathrm{SOH}$ character (Start of Header)

(3) Address character (using address of RPD to which this response pertains)

(4) Status character (ACK, NAK, or Busy)

(5) $\operatorname{STX}($ Start of Text)

(6) ETX (End of Text)

(7) Longitudinal Parity Character position (transmitted as all one's) 
When the SCTS (Station Clear to Send) signal is received, is is applied to the Bit/Character Counter (2.4.2), together with the SXCL (Station Transmit Clock) signal. The Bit/ Character Counter develops bit and character pulses which are used to step through the response message. The Character Counter is used to furnish character addresses to the ROM, which puts out 8 -bit characters ( 7 bits plus parity). The Output Multiplexers (2.4.1) are used to serialize the characters and to merge the address of the pertinent RPD into the fixed-format message at the appropriate character position. The address to be used in the first acknowledgement is obtained from the second buffer register. The ROM has the capacity for four bullt-in messages, three of which are used: ACK, NAK, and Busy. The difference is in the code for the status character, which corresponds to the above conditions. The selection is made by properly energizing the high-order address bits of the ROM, to select the quadrant containing the desired message.

\subsection{Generation of Second Acknowledgement}

The second acknowledgement is generated in a manner similar to that described above, except that only an ACK response can be generated at this time. When an RPD has successfully completed the printing operation for a message previously received from the computer, it sends an ACK signal to an associated flipflop in the RPSC (ACK Storage Flipflops) $(2 \cdot 4 \cdot 5 \cdot 0-N)$. There is a flipflop for each RPD, and it is possible that more than one of these flipflops might be on at the same time. If more than one is on, the response messages are generated by starting with the lowest-numbered RPD as determined by the ACK Priority Determiner(2.4.5). As in the case of the first acknowledgement, a signal is sent to the Transmit Priority Determiner (2.4.3). This resolves any conflict in case there is a pending first acknowledgement request at the time that the second acknowledgement request occurs. If there is such a conflict, the first acknowledgement is given priority. When the second acknowledgement is to be sent, the Transmit Priority Determiner sends an RTS signal to the Transparency Unit. When the computer is ready to receive data, the SCTS signal is sent to the RPSC, and this initiates the response message. The fixed format portion is read out of the ROM with the status character containing the ACK code. The address which is inserted in the address character is obtained by sampling the address thumbwheel switches for the specific RPD, via the address switch multiplexers $(2.4 .6)$.

\subsection{RPSC Annex}

The RPSC was originally designed to drive 8 RPDs. This was 
later updated to 12 RPDs by adding another unit, called the RPSC annex. This contains additional receptacles, thumbwheel switches, and associated logic. Extra driving circuitry in the RPSC is also included to accommodate the increased number of loads.

\subsection{RPSC DETAILED DESCRIPTION OF LOGIC CIRCUITRY}

\subsection{Data Flow (Drawings 2.2.1 - 2.2.7)--Character Shift Register (Dwg. 2.2.1)}

Data comes to the RPSC from the computer via the Transparency Unit as a serial bit stream. Characters are received in an eight-bit shift register. Data passes through the shift register and is routed to the appropriate RPD, as designated in the message address. The output stage of the shift register (BO) feeds a set of line drivers because of the number of loads which must be driven (12 RPDs have been employed).

The received data enters the shift register through a gate which can inhibit the reception of data if the controller becomes overloaded. This would occur if there were two previous messages for which first acknowledgements had not yet been sent. The BFINH (Buffer Inhibit) signal is used for this purpose. Also, the input to the shift register is inhibited for the character following ETX, which is the last data character of a message. The Longitudinal Parity character would be expected to follow ETX. However, in the DDES system this character is simply transmitted as all one's. The RPSC, therefore, blocks out this character and instead substitutes a Longitudinal Parity character of its own.

The Received Clock signal is used to drive the shift inputs of the shift register, after shaping and timing. This signal consists of a continuous stream of bit pulses during recelpt of a message. It also feeds a set of line drivers to provide the CL (clock) signal to the rest of the system.

The shift register supplies characters, eight bits in parallel, to several places, which are discussed elsewhere. One of these is the Longitudinal Parity Generator. After the last data character is transmitted, the Longitudinal Parity character is received from the Longitudinal Parity Generator, eight bits in parallel, and is strobed into the shift register with the PE (Parallel Entry) signal.

\section{Longitudinal Parity Generator (Dwg. 2.2.2)}

This consists of eight toggle flipflops which have both 
their inputs and outputs connected to corresponding stages of the Character Shift Register. The parity generator is cleared prior to the receipt of a message, by the ICR (Initial Condition Reset) signal. As each character appears in the shift register, the L.P.Str. (Longitudinal Parity Strobe) signal causes the bits of the character to be applied to the toggle inputs of the parity generator flipflops. After the ETX character has passed through, the contents of the parity generator are entered, eight bits in parallel, into the shift register for transmission as the last character of the message. The sense of the longitudinal parity is even. That is, the total number of one's occurring in any bit position should be an even number, when taken over a complete message, including the parity character.

\section{Character Decoders (Dwg. 2.2.3.1)}

The RPSC must recognize a number of different characters, for control purposes, and this is done by using a palr of decoders which are connected to appropriate gates. Two hexadecimal decoders are used. The low order four bits (BO through B3) are connected to the minor decoder which provides signals MNO through MN15. The high order three bits (B4 through B6) are connected to the major decoder, which provides signals MJO through MJ15. One input to this decoder is grounded, since it is unused.

\section{Character Recognizer (Dwg. 2.2.3.2)}

This consists of a set of gates which are driven by signals from the Character Decoders and which furnish outputs corresponding to the various special characters:

$\begin{array}{ll}\text { SOH } & \text { (Start of Header) } \\ \text { STX (Start of Text) } \\ \text { ETX (End of Text) } \\ \text { SYN (Synchronizing Character) } \\ \text { CF (Printer Command Flag) } \\ \text { NUL (Null) } \\ \text { DEL (Delete) }\end{array}$

Message Address Register (Dwg. 2.2.4)

This is an elght-bit register which is loaded in parallel from the Character Shift Register when the address character for a message is received. A message for the RPSC will have $a$ one in $B 6$ and a zero in $B 5$ of the address character. When this combination is recognized, the address 
character is loaded into the address register. Whenever an address is loaded into the register, the eighth bit (which is not used for the address) is filled with a one. The presence of a one in this location is used as a signal that an address is present and this signal is used to enable a set of address comparators. The signal is designated EnCmp. (Enable Comparators). The five low order bits of the address are applied to a set of drivers, the outputs of which are designated CAO through CA4. These signals go to the address comparators.

Address Comparators, RPDO through RPD7 (Drawings 2.2.5 $2.2 .5 .7)$

Each RPD is assigned an address by means of thumbwheel switches, so that RPD addresses can be reassigned, should the occasion arise to do so. These switches provide one set of inputs to the set of address comparators, the other set coming from the Message Address Register, signals CAO through CA5. A comparison is made at the time that an address is loaded into the address register.

The comparator for which an address match is obtained will energize its output signal, which is designated the SEL. " $N$ " signal (Select RPD "N", where " $N$ " is the identifying number of the desired RPD.) If the DTR signal for the selected RPD is not on, or if there is an acknowledgement message awaiting transmission for the selected RPD (AKI " $N$ " signal present), an " $N$ " IGN signal will be generated at this time (Ignore RPD "N"). If the selected RPD is busy (BZ signal present), an "N" BZ signal will be generated (RPD "N" Busy).

\section{Line Drivers (Drawings 2.2.6 - 2.2.6.7)}

The Sel. "N" (Select RPD "N") signal is used to gate a set of output signals from the RPSC to the selected RPD. There are six output signals to each RPD. Of these, the Data and CL. (Clock) signals are present at all times and are not gated. (They simply would not be acted upon by an RPD unless the other signals were present.) Control Lines 1 and 2 and the X ChCl signal are gated with the Sel. "N" signal. ( $\mathrm{X} \mathrm{Ch.Cl}$. is the Transmitted Character Clock, which serves to delineate characters.) The Sel. "N" pulse is gated with a signal designated AØ3 (After Phase Three) which comes on after the address of a message has been recognized and remains on for the balance of a message. The output of this gate is the Select signal. This activates the selected RPD. 
The set of "N"IGN signals are ORed together to produce the NoAd. (No address) signal, which means that the RPSC is not to take action on that address (since the corresponding RPD is not in a ready state).

The set of "N"BZ signals are ORed together to produce the SBZ (Selected Station Busy) signal, which means that the RPD designated in a message is not free to receive the message.

\subsection{RPSC CONTROL CIRCUITS (Drawings 2.3.1 - 2.3.7)}

Phase Advance Generator (Dwg. 2.3.1)

Operation of the RPSC during the receipt of a message from the computer is divided into phases. The phases progress as follows:

\section{$\underline{\text { State }}$}

$\emptyset 0:$

$\emptyset 1:$

$\emptyset 2:$

$\emptyset 3:$

$\emptyset 4:$

$\emptyset 5:$

$\emptyset 6:$

$\emptyset 7:$

\section{Conditions for advancing phase}

Expect SYN, advance to $\emptyset 1$ after it occurs

Expect SYN, advance to $\emptyset 2$

Ignore further SYNs;

Expect $\mathrm{SOH}$, advance to $\emptyset 3$

Interpret address character;

if designated station is not busy, advance to $\emptyset 4$

Expect STX, advance to $\emptyset 5$

Message occurs in this phase, between STX and ETX; Expect ETXD and advance to $\emptyset 6$

Advance to $\emptyset 7$

Generate FIN (Message Finished) Signal

The Phase Advance Generator develops a set of pulses for advancing the phase counter in accordance with the above schedule. The Phase Advance Generator produces the Adv $\emptyset$ (advance phase) signal under the following conditions:

$\emptyset 0$ and SYN

$\emptyset 1$ and $S Y N$ 
$\emptyset 2$ and $\mathrm{SOH}$

$\emptyset 3$ and SBZ - (Selected Station not busy)

$\emptyset 4$ and STX

$\emptyset 5$ and ETXD

$\emptyset 6$

The above signals are oRed and applied to the D input of a flipflop which has the ChCl- (Character Clock inverted) signal applied to its clock input.* When this flipflop goes on, it applies an "on" signal to the D input of a second flipflop which is clocked with CL- (BIt Pulses, inverted). The second flipflop thus is turned on at the first bit time after the character pulse. The output of this flipflop is the Adv $\emptyset$ signal, and the inverse of this signal is used to reset the first flipflop. Once the first flipflop is reset, it applies an "off" signal to the D input of the second flipflop, which then goes off at the next bit pulse.

Sequence Checker (Dwg. 2.3.2)

This circuit contains a set of gates which monitor for conditions that would represent violations of the sequence rules previously described, and also detects various special conditions that can occur in connection with incoming messages. An Abort signal will be generated under any of the following conditions:

SBZ and $\emptyset 3:$ Selected Station Busy at Phase 3.

SOH and Not $\emptyset 2$ : SOH at any time other than Phase 2.

STX and Not $\emptyset 4$ : STX at any time other than Phase 4.

(CF or ETX) and Not $\emptyset 5$ :

Printer Command Flag or ETX at any time other than Phase 5.

ETX and Not 4CF: ETX occurs without four Printer Command Flags having occurred.

SYN and (Not $\emptyset 1, \quad$ SYN occurs at a time other than Phases 1,2 or 6

Not $\emptyset 2$, or Not

$\emptyset 6)$ :

A D-type flipflop w111 assume the state applied to the D input upon the occurrence of a clock pulse. 
An Abort Signal will cause Ld: Bfr.1 (Load Buffer 1) signal to be produced, which will cause the address contained in the message from the computer to be loaded into the first address buffer register. This will result in the generation of a response message by the RPSC. If the selected RPD was found to be busy (SBZ on) during Phase 3, a DBZ (Detected Busy) signal will be produced, and this will cause the response message to have its status character set to the code for Busy. If the Abort was caused for any other reason, a DNAK (Detected NAK) signal will be produced, and this will cause the response message to have its status character set to the code for NAK.

Assuming that a message has been recelved successfully, the Busy signal for the selected RPD (SBZ signal is checked at Phase 7. If the SBZ signal is on, it signifies that the RPD is busy processing the message just received, which is the desired situation. This sets a flipflop which in turn will produce the Ld. Bfr. I signal. In this case, since there was no Abort signal, an ACK response message will be generated. This is the first acknowledgement message. If the selected RPD is not found to be busy at this time, the Abort flipflop is turned on. This will lead to a DNAK signal being produced, and a NAK response message will be sent.

A Ld. Bfr. 1 signal triggers a one-shot which generates the FIN (Message Finished) signal.

The Abort flipflop is reset by the ICR (Initial Condition Reset) signal and also by the ETXD signal.

Phase Counter and Decoder (Dwg. 2.3.3)

The phase counter has three stages and drives an octal decoder which produces eight outputs: $\$ 0$ - through $\$ 7$-. These are inverted to produce $\emptyset 0$ through $\emptyset 7$. The phase counter is driven by the Adv. $\emptyset$ signal from the Phase Advance Generator. The address of an incoming message is received during Phase 3, after which a variety of activities occur. Because of this, a flipflop is set at the start of Phase 4 and remains on until the ICR signal occurs. The output of the flipflop is applied to several line drivers which produce the AØ3 (After Phase 3) signal.

The phase counter is reset by the $R \emptyset C$ (Reset Phase Counter) signal which can occur for a variety of reasons, as discussed in the next section. When the phase counter is in Phase 0 , it causes the ICR (Initial Condition Reset) signal to be produced. 
This circuit develops the $\mathrm{R} \emptyset \mathrm{C}$ (Reset Phase Counter) signal in response to a variety of conditions:

$\emptyset 1$ and Not SYN:

$\emptyset 2$ and Not (SYN or SOH):

$\emptyset 3$ and No Ad. :
Any character other than SYN at Phase 1

Any character other than SYN or SOH at Phase 2

No Address signal at Phase 3

(Addressed RPD not in Ready State)

These signals are gated together and applied to the D input of a flipflop which is clocked by the ChC1-signal. This flipflop is reset by the ICR signal. The output of this flipflop is ORed with the FIN-signal and Master Reset signal.

Bit Counter and Character Clock Generator (Dwg. 2.3.5)

The bit counter consists of three stages driven by the $\mathrm{Cl}$. signal (Bit Pulses), and counts off bits in groups of eight. A fourth stage is separately driven by the inverted $\mathrm{Cl}$. signal. The outputs of these stages are gated to produce the $\mathrm{X} \mathrm{Ch}$. Cl. (Transmitted Character Clock) signal, which is a pulse that occurs during the last half of the seventh bit interval. This in turn triggers a flipflop which produces the Ch. Cl. and Ch. Cl-signals. This flipflop is reset by the next bit pulse which occurs after it is turned on, therefore it exists for the first half of the eighth bit interval.

ETXD Detector (Dwg. 2.3.6)

This circuit produces the ETXD (ETX Detected) signal, which indicates the last data character of a message from the computer. The ETX signal from the character recognizer is gated together with the $\emptyset 5$ signal and the $4 \mathrm{CF}$ (Four Printer Command Flags) signal. The output of this gate is applied to the D input of a flipflop which is clocked with the CHPI- signal. This flipflop produces the ETXD signal and its inverse. Following the occurrence of the ETX character, the Longitudinal Parity character is to be entered into the Character Shift Register and transmitted. The PE- (Parallel Entry-) signal is used for this, and is produced by gating together the ETXD signal, the Cl. signal, and signals from the stages of the bit counter. The output of this gate sets a flipflop which is clocked by the ChCl- signal. This flipflop and the ETXD flipflop are initially held in the reset state by the ICR signal. 
This circuitry monitors for a variety of special conditions, drives Control lines 1 and 2 which go to the RPDs, and produces various other signals. The coding of signals on the control lines is as follows:

$\begin{array}{lll}\text { CL 1 } & & \text { CL2 } \\ 0 & & 0 \\ 0 & & 1 \\ 1 & & 0 \\ 1 & & 1\end{array}$

Condition

If there is no special condition present, Control Lines 1 and 2 will be in their zero state, meaning that data is being transmitted to the RPD. When a Printer Command Flag occurs, the CF signal appears and is applied to a flipflop which is clocked with the ChCl- signal. The signal from this flipflop passes through two more flipflops for delay and shaping and then goes to an $O R$ gate which drives Control Line 2. In addition, the CF signal is inverted and passed through several stages of logic where it eventually is used to inhibit the X Ch.C1. (Transmitted Character Clock) for one character interval.

The occurrence of Printer Command Flags is counted by a counter which generates the 4CF (Four Command Flags) signal. (Each message is required to have four Printer Command Flags, delineating the four segments of a message.)

The Longitudinal Parity character is transmitted during Phase 6 . The $\emptyset 6$ signal is applied to an OR gate which drives Control Line 1. A signal on Control Line 1 indicates to the RPD that the Longitudinal Parity character is being sent.

If an Abort signal occurs, it is applied to an OR gate driving Contro1 Lines 1 and 2, thus turning both of these lines on.

The Longitudinal Parity Character is built up by applying successive message characters to the set of flipflops in the Longitudinal Parity Generator. Not all characters are applied, since some of these are intercepted by the RPSC and are not passed along to the RPD. The LPStr. (Longitudinal Parity Strobe) signal is used to apply the desired characters to the Longitudinal Parity Generator. This signal is obtained by using the ChCl signal to drive a one-shot which produces the ChP1 (Character Pulse) signal. This in turn is gated with various signals to inhibit its 
occurrence when it is not desired. It is enabled during Phase 5, which is the interval in which the text portion of a message occurs. It is inhibited for Printer Command Flags, Null characters, Delete characters, and the ETXD signal.

The X Ch Cl (Transmitted Character Clock) signal which is used to drive the RPDs is obtained by using the inverted ChCl signal to clock a flipflop which is enabled during Phase 5, except for the occurrence of Printer Command Flags, Null Characters, and Delete characters. The output of this flipflop is ORed with the $\emptyset 6$ signal to include the Longitudinal Parity interval, and is then combined with the inverted $X$ ChCl signal.

\subsection{RPSC Reply Circuitry (Drawings 2.4.1 - 2.4.6) Output Data Gating (Dwg. 2.4.1)}

The RPSC formulates response messages for transmission to the computer. These messages follow a fixed format, as shown below:

SYN

SYN

Four synchronizing characters

SYN

SYN

$\mathrm{SOH} \quad$ - Start of Heading

Addr. -

Address character containing address of RPD on whose behalf this response is being sent

Status -

STX -

ETX -

LP -
ACK, NAK, or Busy

Start of Text (no text is sent in a response message)

End of Text

Longitudinal Parity Character position, sent as all one's

Response messages are formulated by sequencing through a ROM which has the fixed characters built into it, and merging them with the proper address. For a first acknowledgement, the address is obtained from the second of two address buffer registers; for the second acknowledgement, 
it is obtained by sampling the appropriate thumbwheel switches.

The Output Data Gating consists of multiplexers which recelve characters in parallel form. The various multiplexers are enabled at appropriate times during the message and the applied characters are serialized and merged at an OR-gate for transmission to the computer via the Transparency Unit. A three-stage bit counter (see Dwg. 2.4.2) counts bits in groups of eight for each character. The three stages of this counter ( $\mathrm{BCO}, \mathrm{BCl}, \mathrm{BC} 2)$ are applied to the multiplexers to effect the serializing of characters. The multiplexers contain the decoding circuitry necessary to decode the bit count.

ROM Message Generator (Dwg. 2.4.2)

This circuit contains the ROM (Read-Only Memory) for the fixed portion of response messages, together with the counting and control circuitry for its operation. The ROM is organized into four message areas, three of which are actually used. The two high order bits are used to select the appropriate area for the desired message.

If the response message is to be a first acknowledgement, the status code may be set to ACK, NAK, or Busy. If a second acknowledgement is being sent, it can only be ACK. If a first acknowledgement is being sent, the Xm.Bf. (Transmit from Buffer) signal will be present. This is applied to a pair of gates, together with the NAK and BZ signals, and the outputs of these gates are used as the high order address bits for the ROM. If either of these are on, the corresponding message will be chosen; otherwise, the ACK message will be chosen.

The SXC1. (Station Transmit Clock) signal is used to drive a counter consisting of three low order stages and four high order stages. The low order stages count groups of eight bits to delineate characters. The high order stages are connected as a divide-by-ten circuit and are used to count characters and furnish address signals to the ROM. A formbit decoder is connected to recognize the fifth state of the counter (actually the sixth character). It produces the Sel Rom signal, which causes the address character for the ROM message to be selected. The Sel Rom signal is applied to a pair of gates, together with the Xmt Sta (Transmit Station) signal and the Xmt Bf (Transmit Buffer) signal. The outputs of these gates are the Sel Sta (Select Station) signal and the Sel Bf (Select Buffer) signal. These cause the address character to be selected from the proper source. The Sel Rom signal is used to inhibit the ROM multiplexer 
during the time that the address character is being sent (see Dwg. 2.4.1).

When the character counter has counted ten characters, the response message is completed; at the end of the message, this counter triggers a one-shot which produces the Fin Xmt (Finished Transmitting) signal.

The bit and character counters are normally held in a reset state and are enabled by the presence of the SCTS (Station Clear to Send) signal.

Transmit Priority Determiner (Dwg. 2.4.3)

Requests for the transmission of first and second acknow1edgements can arise independently. This circuit resolves any conflicts which might occur between requests for these two types of response. If the 2 Bf Rdy (Second Buffer Ready) signal is present, it means that a first acknowledgement is to be sent. If the Sta Rdy (Station Ready) signal is present, it means that a second acknowledgement is to be sent. If either of these signals is present, the RTS (Request to Send) signal is sent to the Transparency Unit. The 2 Bf Rdy and Sta Rdy signals are applied to gates which are applied to the $D$ inputs of a pair of flipflops. These flipflops are clocked by the $\mathrm{XmCl}$ signal, using the normal signal on one flipflop and the inverted signal on the other, to avoid the possibility of them being set at the same instant. When either of these flipflops is set, it inhibits the gate leading to the other, thereby holding the other of until the first request has been answered. When the $2 \mathrm{Bf}$ Rdy signal sets its flipflop, the flipflop produces the Xmt.Bf. (Transmit Buffer) signal, meaning that a first acknowledgement signal is to be sent using the address contained in the second buffer register. When the Sta Rdy signal sets its flipflop, the flipflop produces the Xmt. Sta. (Transmit Station) signal, meaning that a second acknowledgement is to be sent, using the address obtained from the thumbwheel switches for the requesting RPD. When the requested message has been sent, the FinXmt (Transmission Finished) signal is applied to the Transmit Priority Determiner and is passed through gates which reset whichever flipflop had been set by the transmission request. The Fin.Xmt. signal is also gated to produce either the Clear 2 Bf (Clear Second Buffer) signal or the Clear Priority Request signal.

Output Buffers (Dwg. 2.4.4.1)

This circuit consists of the first and second buffer register, which receives addresses to be used in first 
acknowledgement messages awaiting transmission. Addresses are transferred in parallel ( 5 bits) from the Message Address Register (2.2.4) into the first buffer. As soon as the second buffer is avallable, an address stored in the first buffer will be transferred to the second buffer, from which it will be picked up during the transmission of a response message. The buffers are actually elght-bit registers, the five low order bits being used for addresses, and the other three being used for status information. The sixth bit is turned on if a NAK response is to be sent; the seventh bit is turned on if a Busy response is to be sent. The eighth bit is turned on whenever an address is transferred into the buffer, and serves to indicate that an address is present. This produces the I Bf Rdy (First Buffer Ready) signal. When an address is transferred from the first buffer to the second, these status bits are transferred along with it. The first buffer is loaded by means of the Ld Bfr 1 (Load Buffer 1) signal applied to its transfer inputs. The contents of the first buffer are transferred to the second buffer by a signal designated strobe Bf 2. This is applied to the transfer inputs of the second buffer via a pair of gates which are enabled by the presence of a one in the eithth bit of the first buffer. If both buffers become full, the input to the RPSC is to be inhibited, since there is no provision for storing any further addresses. This condition is sensed by applying the 1 Bf Rdy (First Buffer Ready) and 2 Bf.Rdy (Second Buffer Ready) signals to an AND gate, the output of which is used to inhibit the data input of the RPSC. This signal is designated BFINH (Buffer Inhibit). A parity generator chip is used to produce the lateral parity bit to be attached to the address character when it is included in a response message.

\section{Output Buffer Control (Dwg. 2.4.4.2)}

Addresses for response messages to be transmitted are first loaded into the first buffer and then transferred to the second buffer when it is available. If the second buffer is not occupied when the first buffer is loaded, this transfer takes place on the next occurring pulse of the Xmt. Clock. If the second buffer is occupied, the transfer does not take place until it becomes available; the transfer occurs on the next occurring Xmt. Clock pulse after the second buffer is cleared.

The Output Buffer Control circuit contains an AND gate which has as inputs the Ld Bfr 1, 1 Bf Rdy, Clr 2 Bf, and 2 Bf Rdysignals.

If the second buffer is avallable, the 2 Bf Rdy signal will 
be off (no address being stored in second buffer). The inverse of this signal (2 Bf Rdy-) is applied to the AND gate, and will be in the on-state under this condition. When the Ld Bfr 1 signal occurs, the first buffer will be loaded, and this will cause the 1 BF Rdy signal to come on. The Ld Bfr 1 signal is applied to the AND gate as a negative pulse, so that the gate will be inhibited for the duration of the pulse. At the trailing edge of the Ld Bfr I signal the AND gate will produce a signal which passes through an inverter and is applied to the D input of a flipflop. This flipflop is clocked with the Xmt Clock signal, which will turn on the flipflop at the first clock pulse which occurs after the $D$ input goes on. The output of this flipflop enables a pair of AND gates which are driven by opposite phases of the Xmt. Clock signal. The output of one of these gates is the Strobe B2 signa1, while the other becomes the CIr I Bf (Clear First Buffer) signal.

If the second buffer is full at the time the first buffer is loading the 2 Bf Rdy signal will be on, and the inverse of this signal will inhibit the input AND gate. When a CIr 2 Bf signal occurs, the second buffer will be cleared and the 2 Bf Rdy signal will go off, causing its inverse to go on, and enabling the AND gate. The 1 Bf Rdy signal will also be present at this time. The Clr 2 Bf signal is a negative pulse and is used to inhibit the AND gate for its duration. At the trailing edge of the $\mathrm{Clr} 2 \mathrm{Bf}$ signal, the D-type flipflop will be enabled, and the sequence described previously will take place, resulting in the generation of the Strobe B2 and CIr 1 Bf. signals.

\section{ACK Priority Determiner (Dwg. 2.4.5)}

When an RPD successfully completes the printing operation on a message, it generates an ACK signal. This sets a toggle circuit in the RPSC and generates a request for the transmission of an ACK response message (second acknowledgement). There is a toggle circuit for each RPD, and these are connected to a priority determining chip having eight inputs. If one or more inputs are on, the chip generates a three-bit output code for the input having the highest priority. There is also an output signal (GS) which goes on if any of the inputs are energized. The three-bit code is transferred, together with the EO signal, to a four-bit latch chip which retains this code until the corresponding response message is transmitted. The three-bit output code is designated the Priority Code. It is applied to an octal decoder which decodes it back into one of eight lines. This decoded signal is used to reset the toggle which was storing the acknowledgement request, since that information has now been transferred to the four-bit latch. The Priority Code 
When any input to the priority chip is energized, that chip produces the GS signal. This is applied to the D input of a flipflop which is clocked with the Xmt Cl- signal. This flipflop goes to a second flipflop of the same type, so that when the GS signal appears, the first flipflop goes on and then the second. The second flipflop generates the Sta Rdy (Station Ready) signal. The two flipflops together contro1 the input to the four-bit latch. At the end of a response message, a Clear Priority Request signal occurs which resets the two flipflops and also the four-bit latch. A gating circuit is included for the selection of one of two sets of address thumbwheel switches. The Up Sw (Upper Switch) signal selects the upper set of switches (original set of eight). The Lo Sw (Lower Switch) signal selects the lower set of four switches, contained in the RPSC Annex.

\section{ACK Storage Flipflops (Drawings 2.4.5.0 - 2.4.5.7)}

The RPSC contains a toggle circuit for each RPD to store requests for the transmission of second acknowledgements. A palr of cross-connected AND gates are used to form the toggle circuit. The toggle circuit is normally in the reset state and is triggered into the set state by a negative "ACK" pulse from the RPD. The toggle circuit produces the ACK " $N$ " signal, where " $N$ " is the number of the requesting RPD. The "ACK" pulse is also applied to an AND gate controlled by the toggle circuit. The output of the AND gate goes negative at the trailing edge of the "ACK" pulse. This signal is applied to the priority encoder.

\section{Address Switch Multiplexers (Dwg. 2.4.6)}

For second acknowledgement messages, the address character is obtained by sampling the thumbwheel switches for the RPD which initiated the request. Five multiplexers are used for this purpose, one for each of the five low order bits of the address character. All of the " 0 " bits of the elght sets of thumbwheel switches are connected to the first multiplexer chip; all of the " 1 " bits to the second chip, etc. The three-bit priority code from the ACK Priority Determiner (Dwg. 2.4.5) is applied to each of the multiplexers to select the desired one of the eight inputs. The multiplezers have built-in decoders for decoding the three-bit octal selection code. The selected address code is avallable at the output of the multiplexers as five bits in parallel, to which are added three more bits. The two high order address bits are permanently wired as one's. A parity generator chip is used to produce the eighth bit which contains lateral parity. 
A set of drive circuits are used to provide signals to the RPSC Annex which contains additional circuitry for the operation of four more RPDs. 
The RPD Traffic Control Unit

\section{Table of Contents:}

1. Introduction

2.0 The Control Console

2.1 Console Displays

2.2 Audible Alarm

2.3 Console Switches

3.0 The RPD Electronics-Functional Description

3.1 Communications

3.2 The RPD Memory

3.3 Control Console Logic

3.4 Alarms

4.0 The Remittance Handler and Printing Systems

4.1 Paper Handling

4.2 Check Printing

4.3 The Endorsement

4.4 Alarms

5.0 The Document Handler and Printing System

5.1 The Document

5.2 The Document Label

5.3 Alarm Messages 
6.0 The 813 Register

6.1 Register Messages

6.2 Register Printer

6.3 Printer Alarms

7.0 Audio Jackbox

8.0 RPD Logic Control Circuitry

8.1 Physical Description

8.2 Theory of Operation

8.3 KST

8.4 RPD Control Logic

8.5 RPD Busy - Enable (Dwg. 3.1.1)

8.6 Clear Memory Signals (Dwg. 3.1.2)

8.7 Special Command Recognizer (Dwg. 3.1.3)

8.8 Longitudinal Parity Checker (Dwg. 3.1.4)

8.9 Character Shift Register (Dwg. 3.1.5)

8.10 Printer Select Character Decoder (Dwg. 3.1.6)

8.11 Lateral Parity Checker (Dwg. 3.1.7)

8.12 OK to Print (Dwg. 3.1.8)

8.13 Internal Clock Pulse Divider Chain (Dwg. 3.1.9)

8.14 Minor and Major Bits Decoders (Dwg. 3.1.10)

8.15 Control Character Recognizer (Dwg. 3.1.11)

8.16 Data Bit Serializer (Dwg. 3.1.12) 
Section III

Table of Contents (Continued)

9.0 Teletype Buffer and Control Logic

9.1 Teletype Enable Circuit (Dwg. 3.2.1)

9.2 Address Counter for Memory Chips (Dwg. 3.2.2)

9.3 Teletype Storage Chips, Bit 0 through 6 (Dwgs 3.2.3ฤ-3.2.3.6)

9.4 Teletype Transmit - Receive Chip (Dwg. 3.2.4)

9.5 Teletype OK (Dwg. 3.2.5)

9.6 Forced Form Feed Generator (Dwg. 3.2.6)

9.7 Special Character Decoder (Dwg. 3.2.7)

9.8 Forced Form Feed Insertion (Dwg. 3.2.8)

9.9 Ribbon Motion Detector (Dwg. 3.2.9)

9.10 Teletype Special Conditions (Dwg. 3.2.10)

9.11 Document Printer Enable (Dwg. 3.4.1)

9.12 Document Clock Generator (Dwg. 3.4.2)

9.13 Document Data Storage Chip (Dwg. 3.4.3)

9.14 Document Data Deserializer (Dwg. 3.4.4)

9.15 Document Address Counter (Dwg. 3.4.5)

9.16 Document Printer Drivers (Dwg. 3.4.6)

9.17 Document Printer OK (Dwg. 3.4.7)

10.0 Amount and Audit Trail Printing

10.1 MICR (AMOUNT) Printer Enable (Dwg. 3.5.1)

10.2 MICR (AMOUNT) Clock Generator (Dwg. 3.5.2)

10.3 MICR Address Counter (Dwg. 3.5.3)

10.4 MICR Storage Chip (Dwg. 3.5.4) 
10.5 MICR Deserializer (Dwg. 3.5.5.1)

10.6 MICR Data Drivers (Dwg. 3.5.5.2)

10.7 MICR OK (Dwg. 3.5.6)

10.8 MICR Status and End Signals (Dwg. 3.5.7)

10.9 Burroughs Event Signals (Dwg. 3.5.8)

11.0 Audit Trail Printing on Burroughs Printer (Drawings3.5.9 3.5.15)

11.1 Drawing 3.5.9

11.2 Drawing 3.5.10

11.3 Drawing 3.5.11

11.4 Drawing 3.5.12

11.5 Drawing 3.5.13

11.6 Drawing 3.5.14

11.7 Drawing 3.5.15 


\subsection{INTRODUCTION}

A work station within the RPS system is known as a Remittance Processing Device (RPD) and is a desk-like enclosure suitable to the environment of a large clerical office and contains the necessary electronic, electromechanical and mechanical equipment necessary to receive and store messages from the RPSC and to print and apply them to remittances and documents as required by the RPS.

The RPD consists of the following units resting on or enclosed in the structure which also provides workspace for a Second Entry Operator (SEO):

a. A keyboard and CRT display unit (KST) connected to the DDES computer system through a DCU, which the SEO used for data verification operations;

b. The Control Console;

c. The RPD electronics for interacting with the RPSC, for receiving and storing messages for the printers, for controlling the printers and paper handlers, and for interacting with the Control Console;

d. The Remittance Handler and Printing System;

e. The Document Handler and Printing System;

f. The Register Printing System;

g. The RPD Power Supplies;

h. The Audio Jackbox

\subsection{THE CONTROL CONSOLE}

\subsection{Console Displays}

The console displays include provision for the following displays:

a. A 12-element projection display unit composed to display three-line messages with 3/8-inch high characters as follows:
(1) Top Line: CHECK MICR LABEL or 813
(2) Center Line: PAPER RIBBON or a blank
(3) Bottom Line: LOW OUT JAM STOP 
(4) Background: YELLOW, RED, or BLACK

The characters may be white on whichever background is selected. Any combination of one message from each line and any background color is selectable.

b. GO light: A green light indicates when a message has been received for printing.

c. Manual light: A red light indicates when the RPD is in the manual mode. This light is incorporated in the Manual switch.

d. Power light: An amber light is on whenever all the units of the RPD are on. Generally this shall be the only light lit on the Control Console when the RPD is idle.

Appropriate alarm messages are displayed on the console display when there is a malfunction in one of the RPD units or replenishment of some supply is required. These are reset whenever the malfunction is corrected or the material supplied.

\subsection{Audible Alarm}

An audible signal with a frequency of about $400 \mathrm{~Hz}$ and manually adjustable volume is included in the control console to indicate to the SEO that a remittance or document required for the printing of the message is not in position. A volume control is provided for limited adjustment of the audible level.

\subsection{Console Switches}

Switches are provided for the following functions:

a. Mode Selection--This switch provides means of switching back and forth between the Normal (machine-controlled) and the Manual modes of operation. While in the Normal mode, all other Console Switches are disabled except those for Lamp Test.

b. Unit Selection--It is possible to select for control the printing of any of the four messages, together with the operation of the necessary paper handler. It is also possible to select all of the four messages at the same time. A non-selecting position is also available. 
c. Operation Selection--A set of push-button switches are provided to cause the following operations to occur when Manual and a message are selected:

(1) Proceed--Continue printing or paper moving from a point of interruption;

(2) Repeat--Repeat the entire printing cycle for the selected message;

(3) Cancel--Set to the conditions which would have been reached at the end of the printing cycle without causing any printing or paper motion.

d. Lamp Test--A set of switches is provided to permit operation of the display lamps, the panel lights, and the buzzer in such a way that it is possible to determine whether any one is out of order.

Physical and Circuit Descriptions of the Control Console are shown in Section 7 of this report.

\subsection{THE RPD ELECTRONICS--FUNCTIONAL DESCRIPTION}

The RPD Electronics consists of the three major functional parts:

(a) The communications Electronics

(b) The Message Storage Electronics

(c) The Control Console Circuitry

\subsection{Communications}

The Communications Electronics performs the following functions:

(a) Receives commands from the RPD Interface

(b) Receives messages from the RPD Interface

(c) Performs format checks on the messages

(d) Sends status signals to the RPD Interface

(e) Diverts message sections to the proper portion of the Message Storage. 
The commands recognized come from the RPSC and are:

(a) A reset or abort command to cause the RPD to go to the idle state;

(b) A selection command to prepare the RPD to receive message data and data control commands;

(c) The next character is a data control command;

(d) The next character is a longitudinal parity character.

Status messages to the RPSC from the RPDs indicate their being in one of the following states:

(a) Inoperative (No DTR)

(b) Idle (DTR)

(c) Busy

(d) Aborting the message

Both 1ateral and longitudinal parity checks are made by the RPD. Checks for a valid memory selector character are also made. Failure to meet any of these checks causes an abort status signal to be sent to the RPD Interface and to the control portions of the RPD electronics.

When a Command Follows signal is received from the RPSC, the next character is taken as a control character to determine where the following message shall be stored, as follows:

@--Deselect memory, do not store any following message

A--Store the following message in the MICR memory

B- Store the following message in the Check memory

D--Store the following message in the Label memory

H--Store the following message in the 813 memory

Any Other --Invalid, cause an abort signal to be generated.

When an LP Character Follows signal is received from the RPSC, the RPD disables the lateral parity check and compares the next character with the longitudinal parity character generated during reception of the message. If the two do not agree, an abort signal is generated. If they do agree, the Go light on the Control Console is lighted. 
When a Select signal is received, the status signal changes from the Idle to the Busy condition. When all printing has been completed, the signal changes back to the Idle condition. After an abort signal has caused the proper control resets, the status reverts to the Idle condition.

\subsection{The RPD Memory}

The RPD Memory consists of four sections, one for each of the printer messages. Each section is large enough to store a maximum length printer message, with enough bits per character to be able to select the proper symbol set. Selection of the section of memory to load is controlled by the Communications Electronics.

The memory is cleared at Selection signal time. It is loaded at a rate consistent with the 3520 bps incoming message rate. The message is stored until it is to be replaced by the next message.

The memory is under control of the individual printer control circuitry. The reading rates are consistent with the printing rates of the individual printers.

The output memory address register(s) are set to the initial address of the message section at clearing time, or at a Repeat signal when in the Manual mode. If there is an interruption to the printing because of a malfunction, reading will continue from the next address when a Proceed signal is received.

\subsection{Control Console Logic}

This circuitry accepts the input alarm signals from various units of the RPD and converts them into signals to cause the appropriate message to appear on the console display. It controls the GO and Power lights on the console.

This circuitry also accepts the switch signals from the Control Console and causes the selected operations to occur.

\subsection{Alarms}

The RPD Electronics unit monitors its power supply voltages and causes the Out of Service status to occur, and the Power light to go out, if any of its power supplies fail.

As soon as a message from the RPSC has been accepted, the GO light will turn on. If a remittance message is to be printed, but no remittance is placed in the entry point within 20 seconds after the GO light is turned on, the Console audible 
alarm will sound for 5 seconds. If a document message is to be printed, and a document is not in the entry position within 20 seconds after the GO light is turned on, the audible alarm sounds for 5 seconds, and the YELLOW background lights up on the display. Both signals cease as soon as the remittance and/or document is placed in the entry point(s), or the activating message is cancelled manually.

\subsection{THE REMITTANCE HANDLER AND PRINTING SYSTEM}

The Remittance Handler and Printing System is a modified Burroughs S102/S103 Electronic Proof Encoder described in Section IV of this report. Its functions in the RPD are as follows:

(a) Take the remittance from the entry point, cause the messages described below to be printed, and stack the remittances convenient for pickup by a clerk.

(b) Print a numeric audit trail on the top back edge of the remittance.

(c) Print according to ABA standards the amount of the remittance in MICR characters on the lower right front edge of the remittance.

(d) Print an endorsement located centrally vertically near the right end on the back of the remittance.

(e) Cause the appropriate alarms to be displayed on the Control Console when malfunctions occur or when any supply needs replenishment.

\subsection{Paper Hand1ing}

The SEO has only to place the remittance in the entry position to cause all required remittance operations to take place. The remittance will pass through the Check, MICR and Endorsement printing stations and be stacked in a convenient position for pickup. It is capable of transporting any rectangular check, money order, or remittance slip with maximum $A B A$ height, and printed on any good quality paper stock from 15-1b (500 - 17 × 22 basis) to punchcard stock with punches and rounded corners.

A remittance will pass through the system while printing maximum length messages at all stations within approximately 10 seconds after being placed in the entry point with the CO light on.

A remittance amount of 10 numeric characters, preceded and followed by the ABA amount symbol, is printed on the lower 
right front edge of the remittance in accordance with the provisions of American Bankers Association Document 147R3, The Common Machine Language for Mechanized Check Handling.

The stored message to produce this message is a variable length amount (maximum of 10 characters) followed by a breaker (+) symbol sent to the RPD MICR memory. In the printing process, leading zeros are filled in as needed. If only the breaker symbol has been sent, the amount printed will be 10 zeros.

\subsection{Check Printing}

An audit trail is printed upside down within 1/2-inch of the top edge of the remittance on its back. Except for the Pointer Symbol, all characters are numerics. The code for transmitting the pointer symbol from the computer is the ASCII code for ; . The printed message is arranged as follows :
a. 1 Pointer
b. 9 Numerics
c. 1 Space
d. 4 Numerics
e. 1 Space
f. 1 Pointer
g. 3 Numerics
h. 1 Space
1. 14 Numerics
j. 1 Pointer

These shall be printed in one line in MICR characters. The use of the same printer for MICR and CHECK printing is made possible by use of the modified Burroughs S102/S103 Electronic Proof Encoders.

Location of the printed messages on the remittance are shown on Figure 1. Note that the location of the printed message must not be closer than one inch of the right-hand edge of the remittance. 


\subsection{The Endorsement}

The endorsement consists in part of a fixed text which is alike for all machines at a particular IRS Service Center. The text was provided by the IRS for the Philadelphia installation. It is contained on a rubber stamp which operates in conjunction with a PORLON ink roller and is a part of the Burroughs S102/S103.

The second part is a three-digit number which is settable by the SEO and is assigned to each machine in the Service Center.

This endorsement is to be placed on each remittance passing through the handler without any need for a message from the computer. It is located on the back of the remittance, centered vertically, and within 1/2-inch of the right end of the remittance. It is printed clearly in an ink color selected by IRS for a particular run of work.

The endorsement printer is easily accessible by the SEO for changing the date or replenishing the ink supply or ribbon. The endorser may be manually removed to prevent endorsement, should this be desired, and as a safety precaution while the $\mathrm{SEO}$ is working in the area.

\subsection{Alarms}

Alarm messages appear on the Control Console display whenever a sensed malfunction occurs.

The top line of the display is "CHECK" for malfunctions associated with printing the audit trail, and "MICR" for malfunctions associated with printing the amount.

Failure of paper to move other than because a printer will not accept it, or does not complete printing is indicated by "PAPER" on the second line. For these two exceptions, the second line is left blank.

Failure of paper to move other than in the printers is shown by the word "JAM" in the third line, except when it has passed the last printer, when it shows "STOP".

Failure of a printer to accept the remittance or to complete printing is indicated by "STOP" in the third line.

Should power to the remittance handler or to any of the printers fail, the amber Power light will go off, and the message CHECK OUT with red background will be displayed. 
Should a remittance message be received, and no remittance placed in the entry point of the handler within 20 seconds of that time, the audible alarm on the Control Console will sound for 5 seconds.

\subsection{THE DOCUMENT HANDLER AND PRINTING SYSTEM}

This system is intended to print a message from the computer on a label and to attach the label to the document associated with a remittance. The SEO enters the document into the system, retrieves and stacks it after the label is applied.

The SEO is required to use only one hand for entry and retrieval, and does not have to take particular care about alignment when entering the document.

For a complete description of the document labeling device refer to Section 8 of this report.

\subsection{The Document}

Documents may vary in size from $3-1 / 2$ by 7 to $8-1 / 2$ by 15 inches. They may consist of single sheets of 15-1b. paper to a packet 3/4-inch thick. The document handler can handle this range of sizes in either orientation with thicknesses up to $1 / 2$-inch.

Document thickness may vary across a single document because of attachments by tape, glue, or staples.

\subsection{The Labe1}

The label is approximately 1/2-inch wide, and 4-inches long. The label message consists of three groups of numerics (14 digits, 9 digits, and 10 digits), each separated by a single space. The printed characters are about 0.080 by 0.120 inches, spaced to remain within the maximum label length. The data is printed in a single line.

\subsection{Alarms}

Alarm messages are displayed on the Control Console in case of malfunction or need for replenishment of supplies. These are individualized as much as possible within the restraints of the display so as to help pinpoint the malfunction. An alarm causes the RPD to refuse to accept any more messages until the system is restored to normal, or the operator cancels the message by using the Control Console switches. 
In case of power loss to the handler or printer, the amber Power light will go out, and the message LABEL OUT with red background is displayed.

If a Label message has been received, and a document has not been placed in the entry point within 20 seconds of its reception, the YELLOW background is displayed and the audible alarm sounds for 5 seconds. The alarm turns off when the document is entered.

A signal is given the SEO when the label has been applied and it is proper to remove the document. This signal is a light at each end of the document entry slot which remains on until the document is removed. No new message can be accepted by the RPD until the document is removed.

\subsection{THE 813 REGISTER}

\subsection{Register Messages}

The register form is SUB 813, which is printed in triplicate on blank numbered form sheets. The register for a batch of remittances may take from one to five of these sheets.

All printing, including that for headings, comes from alphanumeric messages from the computer. One message prints the register and column headings; there is a message for each transaction, taking one line; the trailer with batch totals takes another message. The longest printed line is under 60 character spaces in length.

Certain portions of some messages are not to be printed on one copy, these are indicated by codes in the message.

\subsection{The Register Printer}

This unit is a Model 37 Teletype printer and has the following capabilities:

a. It prints on $9-1 / 2$ by 11 inch three-ply continuous forms with a 1/2-inch removable pinfeed strip on each side.

b. Pica-sized numeric and upper case alphabetic characters are required, as well as the symbols ., / and - and Space.

c. The following ASCII function codes must also be recognized: New Line, Horizontal Tabulation, Form Feed, and Escape. The functions of Normal Color Shift and Second Color Shift are recognized by the Teletype codes for these functions. Color shift is a locking function, and does 
not require the function code before each non-normal colored character.

d. The horizontal tabulation stops may be set either by a code sequence or offline using the message generator. (See section $\mathrm{V}$ for details.)

e. Form Feed sets the printing point to 1/2-inch from the top edge of the 11-inch form. Form Feed includes a New Line or Carriage Return operation.

f. Printing and operational rates are such that a maximum form feed operation followed by a maximum line-length message shall take no more than 10 seconds. Horizontal tabulations take no more than spacing time for the same interval.

g. The ribbon is black (normal) and is provided with an uninked section with a barrier between the sections to prevent diffusion of the ink into the uninked portion.

h. Manual New Line and Form Feed operations are possible by use of buttons conveniently located at the printer.

i. An automatic Form Feed signal is generated whenever the printing line moves within I/2-inch of the bottom of an 11-inch sheet.

j. The output of the printer is stacked convenient for pickup by a clerk. The SEO may monitor the printout to see that the printer is working properly, from her operating position.

\subsection{Printer Alarms}

The following alarms are provided by means of the Control Console:

a. 813 PAPER LOW (YELLOW) when the paper supply is sufficient only to finish the current form (no less than five sheets).

b. 813 PAPER OUT (RED) when the printer is writing on the last sheet, or when the supply web has broken.

c. Loss of power will cause the amber Power Lamp to go out, and the message 813 OUT with red background to appear. 
An audio jackbox has been attached to the RPD below tabletop level in a position convenient for the SEO to plug in a set of headphones for training purposes. This circuit is a part of an existing IRS and $C O$ system in the RPS area.

\subsection{RPD LOGIC CONTROL CIRCUITRY}

\subsection{Physical Description}

The logic circuitry for the control functions and message storage are contained in a single electronics drawer located in the left rear corner of the RPD console. The circuit drawings for these functions are the Series 3 drawings appended to this report. Drawing number 3.6.1 shows the component layout and type numbers of the IC chips in this drawer.

\subsection{Theory of Operation}

The overall functional requirements of the Remittance Processing Device and its relationship to other portions of the RPS have been described previously in this report. The following is a description of the detailed functioning of the hardware and logic circuitry.

\subsection{KST}

The KST is electrically independent of the RPD, and has its own communication circuits to and from the DDES computer. Its function is to allow the operator to establish contact with the DDES, to accept Key Verification information, to display the Original Entry information, to direct the operator's attention to any discrepancies, and to allow corrective action to be taken. Upon successful completion of the verification process, the computer formulates a complete print message and transmits this to the RPD, without further use of the KST.

\subsection{RPD Control Logic}

The RPD Control Logic interfaces with the communication circuits from the RPSC. It performs the following functions:

(1) Furnishes a Data Terminal Ready (DTR) signal to the RPSC when it is powered up.

(2) Responds to a signal on the Select line from the RPSC.

(3) Returns a Busy signal to the RPSC when it is processing 98 
a message.

(4) Receives data messages from the RPSC and performs sequence checks, parity, and other error checks on them.

(5) Recognizes special control characters in the data messages.

(6) Monitors the various buffers and status signals associated with the printers.

(7) Decodes the Printer Selection Characters and routes the corresponding message segments to their respective buffers.

(8) Provides an acknowledgement signal to the RPSC upon correct receipt of a print message.

(9) Upon recognition of any error condition, generates an Abort signal which terminates the message reception.

(10) Generates clear memory and reset signals.

The RPD Control Logic receives a set of inputs from the RPSC. These are as follows:

(1) Data--This input is the stream of information bits which occurs when the RPSC routes a message to the designated RPD.

(2) Clock--This input consists of a stream of bit pulses.

(3) Select--This input is energized when the RPSC selects the designated RPD. It comes on just after the address character of a message is decoded by the RPSC and remains on until the transmission of the message is complete.

(4) Control Lines 1 and 2 are inputs energized in various combinations to convey control information from the RPSC to the RPD. They are encoded as follows: 


$\begin{array}{lll}\text { CL1 } & \text { CL2 } & \begin{array}{l}\text { Meaning } \\ 0\end{array} \\ 0 & 1 & \begin{array}{l}\text { Accept Data } \\ \text { Accept Printer Command Code }\end{array} \\ 1 & 0 & \begin{array}{l}\text { Accept Longitudinal Parity } \\ \text { Character }\end{array} \\ 1 & 1 & \text { Abort }\end{array}$

(5) X CH. CL. input is the Transmitted Character Clock and consists of a pulse which identifies the receipt of each character.

The RPD Control Logic develops a set of signals which it transmits to the RPSC. These are as follows:

1. BZ - RPD Busy. This signal is energized by the RPD in response to the Select Signal from the RPSC. It is present through the receipt of a message and persists through the subsequent processing and printing operations.

2. ACK - This is an acknowledgement signal developed by the RPD at the end of the printing operation indicating that the operation has been completed successfully. Note: If the operation does not end successfully, the operator will be notified after a time interval and action must be taken to complete the required operation, so that an ACK is eventually produced.

3. DTR is the Data Terminal Ready signal and signifies that the RPD is powered up and ready for use.

\subsection{RPD Busy-Enable Control (Dwg. 3.1.1)}

When the computer begins to transmit a message, it is first received by the RPSC, which looks for SYN characters, establishes bit and character synchronism, and scans for correct character sequence. When the RPSC detects an $\mathrm{SOH}$ character it accepts the following character as an address character. If the high-order bits are 10, this indicates that the message is addressed to the RPS, and the five loworder bits are decoded to determine which RPD is to be the recipient. The RPSC notes whether the designated RPD is in a Ready status and not busy, and energizes the Select line to it. This sets the Busy flipflop (Dwg. 3.1.1) which in 100 
turn transmits the BZ signal back to the RPSC. When the Busy flipflop is in the reset condition it generates the ICR signal (Initial Condition Reset) which clamps all key circuits in the RPD in a reset state.

When the Busy flipflop is set, this ICR signal is turned off. Note that if the RPD was in manual mode when the RPD was selected, the BZ signal would be inhibited.

Under normal conditions, the Busy flipflop remains on until the message is received and all data is printed, whereupon a NoD signal (no more data) is produced; this triggers a short delay after which the Busy flipflop is reset. A variety of error conditions are ORed together to reset the Busy flipflop at an earlier point, if required, These are as follows:

- Longitudinal parity error

- Lateral parity error

- Abort

- Control character error

- MSTRS (Master Reset)

Upon successful completion of the printing operations, followed by the NoD signal, an ACK signal is produced. This ACK signal will be inhibited if an Abort has occurred, or this can be achieved manually by means of a switch on the operator's control box.

If the RPD is in manual mode and the operator has called for the repetition of any message segment, the Busy flipflop will be inhibited and will not respond to a Select signal.

\subsection{Clear Memory Signals (Dwg. 3.1.2)}

Upon receipt of a message containing data to be stored for each printer, a CLRM signal (clear memory) is produced. This signal is gated with a 96 Kpps signal to produce a short, rapid burst of pulses to clear the storage chip for that printer in anticipation of the new data. The burst of pulses is designated CLRMCl. (Clear Memory Clock). In manual mode, these signals are inhibited so that the previous message data will be stored for reuse. 


\subsection{Special Command Recognizer (Dwg. 3.1.3)}

As described previously, there are two control lines (CL1 and CL2) which convey special commands from the RPSC to the RPD. Signals on these lines are decoded to produce the following signals:

CL1 $=0$, CL2 = 0: Accept data. Under these conditions, both flipflops in the recognizer section remain off and the Ld. Dt. (Load Data) signal is produced.

CL1 $=0$, CL2 = 1: Accept printer select character. Sets command character flipflop, producing CCF signal. This condition exists for one character time. The Ld. CC signal (Load Command Character) is also produced.

$C L=1, C L 2=0:$ Accept Longitudinal Parity Character. This sets the upper flipflop, producing the LGT signal, which prepares the RPD to receive the Longitudinal Parity character.

CLI $=1, \operatorname{CL} 2=1:$ Abort. This combination causes the Abort signal to be produced.

The X Ch. Cl. signal drives a one-shot which produces the Ch. P1. (Character Pulse) signal used throughout the RPD.

\subsection{Longitudinal Parity Checker (Dwg. 3.1.4)}

Longitudinal parity is generated at the RPSC over the span of characters comprising each complete message transmitted to the RPD. (Lateral parity is also generated for each character; this is discussed later). The sense of the longitudinal parity is even; that is, for each bit position the number of one's, taken over all the characters in a message, together with the corresponding bit in the longitudinal parity character, should be even. Each character consists of seven information bits plus a lateral parity bit. The lateral parity bit of the longitudinal parity character represents the longitudinal check for all of the lateral parity bits of the message characters. This may or may not result in correct lateral parity for the longitudinal parity character. (The correct sense of the lateral parity for message characters is odd.) For this reason, lateral parity checking is suspended for the longitudinal parity character. The method of checking longitudinal parity is to apply the successive characters in a message to the toggle inputs of a set of eight flipflops. The set of flipflops is initially held in the reset state by the ICR signal. When a message is to be received, the ICR signal 
is turned off, releasing the flipflops, and the message characters, including the longitudinal parity character, are applied to the toggle inputs. At the end of the message, the set of flipflops is checked with an 8-input AND gate to determine if all of the flipflops have correctly ended up in the reset state. If so, the even longitudinal parity criteria has been satisfied, and no error indication will occur. If any of the flipflops end up in the set state, it indicates the occurrence of an odd number of one's for those bit positions in the message, and at the end of the message an error flipflop will be set, producing the LGPer. signal (Longitudinal Parity error).

\subsection{Character Shift Register (Dwg. 3.1.5)}

An eight-bit shift register is used to receive data characters as they are sent from the RPSC. Characters are received as a serial bit stream. The input to the shift register is the Data signal from the RPSC, and the shift pulses are derived from the $\mathrm{Cl}$. (clock) signal, consisting of a pulse at each bit time. The eight bits are labeled Bt. $\emptyset$ through Bt. 7 with Bt. $\emptyset$ being the lowest-order bit and the first transmitted. The last bit transmitted is Bt. 7, which is the lateral parity bit. The RPSC scans the messages from the computer and screens out certain characters, using these as the basis for special control signals which are sent to the RPD. The characters are not actually inhibited from entering the shift register. Instead, the Data signal and the $\mathrm{Cl}$. signal are always present. However, the response to transmitted characters is always initiated by the X.Ch.Cl. (Transmitted Character Clock) pulses, and these are inhibited by the RPSC, whenever the RPD is to ignore a particular character.

\subsection{Printer Select Character Decoder (Dwg. 3.1.6)}

Messages to the RPD contain four segments for print information, one for each of the four items to be printed (Amount, Audit Trail, Label, TTY Register). Each segment is preceded by the Printer Command Flag and Printer Select Code. The Printer Command Flag is the same for each segment. This flag is recognized by the RPSC, which signals the RPD via Control Lines 1 and 2 that the following character will be the Printer Select Code. Printer selection is governed by the four low-order bits of the Printer Select Code character, as follows:

$$
\begin{gathered}
\text { Bt. } \emptyset \text { - Amount } \\
\text { Bt.1 - Audit Trail } \\
\text { Bt. } 2 \text { - Document Label } \\
103
\end{gathered}
$$


In response to control information from RPSC, the special Command Recognizer develops the Ld. CC signal (Load Command Character) which is sent to the Printer Select Character Decoder. The Decoder contains a four-bit latch which receives the four low-order bits of the Printer Select Code from the Character Shift Register and stores them. For message segments to be printed, there should always be one and only one bit set, and this bit produces an output signal which indicates that the message segment which will follow is to be loaded into the appropriate printer message buffer. If a given message segment is to be disregarded, rather than printed, the Printer Select Code will consist of the "a" symbol, which has all zeros in the low-order bits. Therefore, no buffer will be selected for this segment. An error detection circuit elsewhere monitors the printer selection bits to give an alarm if more than one bit should happen to be set at the same time, which would be an error condition. The four-bit batch is reset by the ICR signal.

\subsection{Lateral Parity Checker (Dwg. 3.1.7)}

Characters are sent to the RPD from the RPSC as a serial bit stream and are loaded into the Character Shift Register. The flipflops of the shift register are attached to the Lateral Parity Checker, which contains the parity check circuitry on one chip. The correct sense for lateral parity is odd, meaning that the number of one's in a character, including the lateral parity bit, should be odd. If the parity check circuitry detects an even number of one's in a character, it puts out a signal which is used to set a flipflop after the character has been received. If this flipflop gets set, it produces the LTPer. signal (Lateral Parity error). This flipflop is reset by the ICR signal.

\subsection{OK to Print (Dwg. 3.1.8)}

The print cycle is initiated upon completion of the receipt of a message by the RPD, provided that there are no indications to the contrary. For each of the four printers there are circuits which monitor the status and produce enabling signals if everything is satisfactory for printing. These are:

$$
\begin{aligned}
& \text { McrEn. - MICR Amount Enable } \\
& \text { ATEn. - Audit Trail Enable } \\
& \text { DcEn. - Document Label Enable } \\
& \text { TTyEn. - Teletype Enable }
\end{aligned}
$$


As long as one or more of these signals is present, it indicates that there is data left to be printed. As soon as all of these signals go off, it indicates that the printing operations are finished, and this results in the generation of the NoD signal (no more data to be printed).

The presence of data to be printed, together with the PrCy (Print Cycle) signal, sets a latch consisting of crossconnected AND gates, the output of which is the OK $\operatorname{Pr}$. signal (OK to Print).

\subsection{Internal Clock Pulse Divider Chain (Dwg. 3.1.9)}

A variety of timing signals are required in the RPD, and these are obtained by dividing down from a $96 \mathrm{kHz}$ oscillator. The signal first passes through a driver which squares it up and produces $96 \mathrm{Kpps}$. This is further divided down to produce 48 Kpps., 9600, 4800, 2400, 240, 30, and 15 pps.

\subsection{Minor and Major Bits Decoders (Dwg. 3.1.10)}

In order to recognize certain special characters, the Character Shift Register is connected to decoders which energize separate output lines for various bit patterns. These are octal decoders, each having three inputs and eight outputs. There is also an enabling input. One decoder is used for the three low-order bits (Bt.0 - Bt.2; Minor Bit Decoder); this decoder is permanently enabled and produces outputs MnO through Mn7. The next three higherorder bits (Bt.3 - Bt.5) are applied to two decoders (Major Bit Decoders). The highest-order information bit is the seventh bit (Bt.6), and this is used to select between the two Major Bit Decoders. When Bt. 6 is zero, outputs Mj 0 through $\mathrm{MJ} 7$ are enabled; when Bt. 6 is one, outputs Mj 8 through $\mathrm{Mj} 15$ are enabled.

\subsection{Control Character Recognizer (Dwg. 3.1.11)}

This consists of circuitry for recognizing various character bit patterns which have special significance to the RPD. This circuitry operates on the outputs from the Minor and Major Bits Decoders. This circuitry also checks the Printer Select code to produce an error indication if more than one printer select bit should happen to be set at the same time.

\subsection{Data Bit Serializer (Dwg. 3.1.12)}

The four message segments to be printed are separately stored on buffer storage chips. One chip is used for the Label printer, one for the Amount and Audit Trail together, 105 
and a set of seven for the Teletype. The storage chips for the Label, Amount, and Audit Trail store only four bits per character (essentially numeric information), and this is stored bit serially on the chips. The Amount and Audit Trail are stored in different regions of the same chip. The Teletype message must be stored as full seven-bit characters. Because these messages may be lengthy, seven chips are used, one for each bit position in a character. The seven Teletype storage chips are loaded in parallel directly from the Character Shift Register.

The storage chips for the Label, Amount, and Audit Trail message segments are loaded using only the four low-order bits (Bt.0 - Bt.3) from the Character Shift Register. This data is serialized by means of a multiplexer chip used in the Data Bit Serializer. Characters are loaded using short bursts of pulses at 96 Kpps., between the recelpt of successive characters by the RPD. When a character is to be serialized for loading, a Ld.Dt. (Load Data) signal occurs. This arms a gating flipflop which is triggered on at the next occurring pulse from the $96 \mathrm{kpps}$ source. This flipflop opens a gate which allows 96 Kpps. pulses to pass through, and these are designated SLd.Cl. (Serial Load Clock). These are applied to a pair of flipflops connected as a counter, which counts off four pulses and then shuts off the gating flipflop. The two counting flipflops also are used as an address generator for the multiplexer, successively selecting Bt.0 through Bt. 3 and presenting these at the output of the multiplexer chip as the Sr.Dt. signal (Serialized Data). This signal goes through gates which apply additional controls and becomes the GSD signal (Gated Serial Data).

One function of the SLd.Cl. signal is to drive a retriggerable one-shot which produces the SLd. signal (Serial Load).

\subsection{TELETYPE BUFFER AND CONTROL LOGIC}

Message segments to be printed on the Teletype are stored in a set of seven storage chips, each representing one bit position In the characters. The Teletype accepts data in the same code that is sent to the RPD; therefore, no code conversion or other transformations are necessary.

The storage chips are cleared prior to the recelpt of a message by holding the data input at the zero level and cycling the memory address counter through all address locations, while applying the Write command $\left(\mathrm{TW}_{\mathrm{r}}\right)$. 
Read-out from the storage chips is non-destructive. The contents of any location selected by the memory address counter will appear at the data output terminal. When data is to be entered, a Write command is applied and the value to be stored (one or zero) is applied to the data input terminal. Immediately after the Write command is removed, the new value can be observed at the output terminal, and may be read as often as desired. In this system, data enters the storage chip at the rate that it is transmitted from the computer. Messages are simply routed through the RPSC to the designated RPD, and the Teletype message segment is routed by the RPD Control Circuitry to the Teletype storage chips.

During the printing of Teletype messages, the data is read out of the storage chips at a rate of 15 characters per second, except for certain special situations in which delays are required, such as a tab command or a form feed. Because seven storage chips are used in parallel for the Teletype data, a full seven-bit character is obtained for each location that is read out. This data must be serialized for transmission to the Teletype, and this is done with a Transmit-Receive chip which contains all of the circuitry for accepting a character in parallel, serializing it, and adding the framing bits.

\subsection{Teletype Enable Circuit (Dwg. 3.2.1)}

The Teletype Enable signal ( $T$ Ty En.) is developed by a pair of cross-connected AND gates used as a latch. It is developed as a result of a Ld. TTy signal, indicating that a message segment has been loaded for the Teletype, or as a result of manually-generated Rpt. TTy signal (Repeat Teletype), indicating that the operator wishes to repeat the printing of the current Teletype message. If there is nothing to inhibit printing, the presence of the TTy. En. signal results in the production of the THRL signal. The THRL signal causes the memory address counter to advance, making characters available at the outputs of the message storage chips; this signal also activates the TransmitReceive chip, sending serialized characters to the Teletype. If a delay is required, a DLS signal (Delay Symbol) occurs and inhibits the transmission for one character time. This is sufficient for the New Line function, but the tab and form feed functions take longer. However, the Teletype has contact closures which exist during these functions, and these are used to provide the additional hold-off time. These are also accompanied by the DLS signal, to allow time for the contact closures to occur.

\subsection{Address Counter for Memory Chips (Dwg. 3.2.2)}

The Teletype address counter contains 8 stages (256 count8) and is used to address the seven storage chips for Teletype 
message segments. It produces Teletype address bits TA through TA7. It operates at three different times, and at different rates:

(1) Prior to receipt of a Teletype message, the address counter cycles through its range while the memory is cleared. It is driven by the CLRM c1. signal at 96 KPPS.

(2) During receipt of a Teletype message segment, the counter counts at a rate of approximately 3500 bits per second, as furnished by the Ld.Dt. signal, in combination with the Ld. TTy. signal.

(3) During printing of a Teletype message, the counter counts at a rate of 15 pps., except when additional delays are needed.

The address counter is reset by the RAAC signal (Reset A11 Address Counters) and also by the Rpt. TTy signal (Repeat Teletype) if the operator wishes to repeat the Teletype message.

9.3 Teletype Storage Chips, Bit 0 through 6 (Dwg. 3.2.3. $3.2 .3 .6)$

These are 256-bit chips, each of which stores a serial bit stream, representing one bit position in the Teletype characters. The chips require an eight-bit address from the memory address register. They require a serial bit stream at the Data In terminal for loading, and produce a serial bit stream at the Data Out terminal. Data is stored by applying a short TWr (Teletype Write) pulse while presenting the input value at the Data In terminal. The bit value at the addressed location is always present at the output (except for a possible transient condition during writing).

\subsection{Teletype Transmit-Receive Chip (Dwg. 3.2.4)}

This chip contains all the circultry necessary to accept the seven bits of a Teletype character in parallel and serialize it, with the proper parity and framing bits added. It is enabled by the TTy, En. signal, and each character is triggered by the THRL signal. The chip derives its bit rate from a 2400 pps. clock signal, which is internally divided by 16 . The chip is capable of both transmitting and receiving, but is only used for transmitting. 
This circuit uses a pair of cross-connected AND gates as a latch, the output of which is the TTy. OK signal (Teletype OK). This signal is turned on by the TTY. En. signal, or if the operator wishes to repeat a Teletype message (TTy. Repeat). However, it is turned off if certain special Teletype conditions are detected, such as Paper Out, or absence of the Data Terminal Ready signal.

\subsection{Forced Form Feed Generator (Dwg. 3.2.6)}

Form feeds are normally included in computer-generated messages, to format the Teletype output as desired. However, a circuit is included to generate a forced form feed command if the printing should come too close to the end of a page. This circuit contains a line counter which keeps track of New Line commands and injects a form feed command into the Teletype message, should one be required. This requires the current printing to be held up, and the form feed character to be inserted in the Teletype character stream. When the line counter reaches a preset value, it generates the FFF signal (Forced Form Feed) which causes the necessary steps to occur.

\subsection{Special Character Decoder (Dwg. 3.2.7)}

Various special characters must be deleted in the Teletype character stream, and this is done through the use of a pair of octal decoders. One decoder operates on bits 0,1 , and 2, and the other operates on bits 3, 4, and 5. Bit 6 is used to inhibit both decoders, since this bit has a value of one for graphics functions and a value of zero for the control functions of interest. For certain functions the DLS signal (Delay Symbo1) is produced. Other characters detected include New Line, Space, and TETX (Teletype End of Text).

\subsection{Forced Form Feed Insertion (Dwg. 3.2.8)}

A pair of four-bit multiplexer chips are used to allow an extra form feed character to be inserted into a Teletype message, whenever required. These chips have two sets of inputs, one set coming from the seven storage chips (TMD-TM6) and the other being wired for the form feed code. The FFF signal is used to select between these two inputs, and to place these signals on the outputs (TD - TD6).

\subsection{Ribbon Motion Detector (Dwg. 3.2.9)}

This circuit was devised to monitor ribbon motion in the 109 
Teletype and to generate a warning signal if no motion was detected after a certain number of characters, spaces excepted. However, this circuit was subsequently deemed to be unnecessary and was disconnected.

\subsection{Teletype Special Conditions (Dwg. 3.2.10)}

A variety of special conditions are indicated by the Teletype mechanism as contact closures. A set of relays and dropping resistors are used to convey this information to the Teletype logic circuitry, in the form of appropriate logic levels.

\subsection{Document Printer Enable (Dwg. 3.4.1)}

The Document (Label) Printer circuitry is enabled by a signal produced by a pair of cross-wired AND gates serving as a latch. The Dc.En. signal (Document Enable) is turned on either by a Ld. Dc. signal (Load Document) from the RPD Control Circuitry, or by a Rpt. Dc. signal (Repeat Document) produced manually by the operator in the event that the printing of the label is to be repeated. The Dc.En. signal is further combined with the DPT signal (Document Print) and the $\mathrm{OK} P r$. signal (OK to Print) to produce the Dc.Pr. signal (Print Document).

\subsection{Document Clock Generator (Dwg. 3.4.2)}

The Dc.Cl. signal (Document Clock) is a pulse train used to drive the address counter for the Document storage chip. It produces three sets of pulses at different times:

(1) A set of pulses at 96 Kpps. resulting from the CLRM C1. signal, to clear the storage chip, prior to receipt of a message.

(2) A set of pulses at approximately 3500 pps. during the loading of a message segment, resulting from the S1d.C1. signal together with the Ld.Dc. signal (Load Document).

(3) Bursts of pulses at $4.8 \mathrm{Kpps}$. when a message is to be printed to shift bits out of the storage chip and send them to the printer.

\subsection{Document Data Storage Chip (Dwg. 3.4.3)}

The Document Data Storage Chip stores a serial bit stream consisting of the message segment for the label printer, using four bits per character. It is filled with zeros prior to the receipt of a new message. It is loaded with 
a new message segment via the GSD signal (Gated Serial Data)

at its input. It produces the Dc.Dt. signal (Document Data)

at its output when a label is to be printed.

\subsection{Document Data Deserializer (Dwg. 3.4.4)}

Data to be printed on the label is retrieved from the storage chip as four-bit serial characters. It enters a four-bit shift register which presents characters to the printer in parallel form. A special code of all one's is used as the stop code and is recognized by an AND gate which produces the DStp signal (Document Stop). Another AND gate recognizes a special code used for a space and produces the Dc.Sp. signal (Document Space).

\subsection{Document Address Counter (Dwg. 3.4.5)}

This is an eight-bit counter (256 counts) which generates addresses DAØ through DA7, for the bits stored in the Document Data Storage Chip. The first two stages of the counter (DA $\emptyset$ and $D A I$ ) are used as a divide-by-four circuit to group the data bits into four-bit characters. A oneshot is triggered at each count of four and generates the D Ch. signal (Document Character). This signal is further combined with the Dc. Pr. signal and D. Stp. signal to produce the Print Command. The counter operates at three different times and at different rates:

(1) Prior to the receipt of a message, the address counter cycles through its range while the memory is cleared, at a rate of 96 Kpps.

(2) During receipt of a message segment to be stored, the counter counts at a rate of approximately 3500 pps.

(3) During the transfer of data to the label printer, the address counter counts in bursts at a rate of 4.8 Kpps.

\subsection{Document Printer Drivers (Dwg. 3.4.6)}

This circuit consists of a set of drivers to convert the logic levels for data and printer commands into suitable levels for transmission to the label printer.

\subsection{Document Printer Enable (Dwg. 3.4.7)}

This circuit contains a pair of cross-wired AND gates connected to form a latch, which produces the Dc OK signal (Document $\mathrm{OK}$ ). The latch can be set by the Dc.En. signal (Document Enable), or by the Rpt. Dc. signal (Document Repeat). 
The circult monitors a set of signals indicating the status of the label printer and will be reset if the DERR signal occurs (Document Error).

\subsection{Amount and Audit Trail Printing}

The amount and audit trail are printed on opposite sides of the remittance (check) by the Burroughs S102/S103 MICR Printer. The amount is printed on the face of the check in the first pass through the printing mechanism. The check then passes between a pair of twisted belts which invert it, after which it passes through the printing mechanism a second time for the printing of the audit trail on the reverse side. An endorsing stamp imprints the reverse side of the check as It passes through the handing mechanism.

The amount and audit trail message segments consist of essentially numeric information and are stored as four-bit characters. They are stored as a serial bit stream, and are stored in a single storage chip. The audit trail segment starts at the beginning of the chip and the amount starts in the last quarter of the chip (two high-order address bits both one's).

Note: It was originally planned to use a separate printer for the audit trail, and this is reflected in a number of the drawings. (See Drawings 3.3.I through 3.3.6.) The separate printer was eliminated in a design rework ("second pass"), and a method was devised for inverting the check and using the Burroughs MICR printer for both the amount and the audit trail.

\subsection{MICR (Amount) Printer Enable (Dwg. 3.5.1)}

The MICR circuitry is enabled by a signal produced by a pair of cross-wired AND gates serving as a latch. The Mcr. En. signal (MICR Enable) is turned on either by a Ld. Mcr. signal (Load MICR) from the RPD Control Circuitry, or by a Rpt. Mcr. signal (Repeat MICR) produced manually by the operator in the event that the printing of the amount is to be repeated. The Mcr. En. signal is further combined with the Mcr. OK signal and the OK Pr. signal (OK to Print) to produce the Mcr. Pr. signal (MICR Print).

\subsection{MICR (Amount) Clock Generator (Dwg. 3.5.2)}

The Mcr. Cl. signal (MICR Clock) is a pulse train used to drive the address counter for the MICR (amount) storage chip. It produces three sets of pulses at different times: 
(1) A set of pulses at $96 \mathrm{Kpps}$, resulting from the CLRM C1. signal, to clear the storage chip, prior to receipt of a message.

(2) A set of pulses at approximately 3500 pps. during the loading of a message segment, resulting from the SId. C1. together with the LDBR signal (Load Burroughs).

(3) Bursts of pulses at $4.8 \mathrm{Kpps}$. when a message is to be printed, to shift bits out of the storage chip and send them to the Burroughs printer.

\subsection{MICR Address Counter (Dwg. 3.5.3)}

The MICR address Counter contains eight stages (256 counts) and is used to address the storage chip which holds the amount and audit trail message segments. It produces address bits MA $\emptyset$ through MA7. It should be noted that the amount message segment is stored in the last quarter of the storage chip, for which the two high-order bits (MA6 and MA7) are both one. These two bits are gated with the LPMCR signal (Load or Print MICR) which causes them to both be one when the amount is being loaded.

The first two stages of the counter (MA $\emptyset$ and MAI) are used as a divide-by-four circuit to group the data bits into four-bit characters. A one-shot is triggered at each count of four and generates the Mch. signal (MICR character).

The counter operates at three different times and at different rates:

(1) Prior to the recelpt of a message, the address counter cycles through its range while the memory is cleared, at a rate of $96 \mathrm{Kpps}$.

(2) During receipt of a message segment to be stored, the counter counts at a rate of approximately 3500 pps.

(3) During the transfer of data to the Burroughs printer, the address counter counts in bursts at a rate of 4.8 Kpps.

\subsection{MICR Storage Chip (Dwg. 3.5.4)}

The MICR Storage Chip stores a serial bit stream made up of the amount and audit trail message characters, using four bits per character. The audit trail is stored in the first part of the chip and the amount is stored in the last quarter, for which bits MA6 and MA7 are one's. 
This circuit contains a four-bit shift register which receives serial data from the MICR storage chip. Four-bit characters are shifted into this register and are available in parallel form at its output. The characters are passed through a multiplexer chip which is used to substitute a special character when an input character of all one's is detected. The output of the multiplexer also consists of four-bit characters in parallel form, signals GMD1, GMD2, GMD4, and GMD8. In addition, a high-order bit is generated, GMD16. An extra bit is obtained by inverting GMD1, and is designated GMDS. This assures that all output characters will have at least a single one, since this indicates the presence of a character to the Burroughs printer. A character pulse is also produced (GMDP) and an MICR Stop signal (McSTP).

\subsection{MICR Data Drivers (Dwg. 3.5.5.2)}

The Burroughs printer used for printing the amount and audit trail was adapted from a unit normally used with a manual keyboard. This circuit contains a set of drivers which simulate the signals that would have been produced by the keyboard. The output signals from the drivers go to a connector that plugs into the receptacle normally used for the keyboard.

\subsection{MICR OK (Dwg. 3.5.6)}

This circuit monitors a set of signals indicating the status of the Burroughs printer. The circuit contains a latch consisting of a pair of cross-wired AND gates, the output of which is the Mcr. OK signal (MICR OK).

\subsection{MICR Status and End Signals (Dwg. 3.5.7)}

This circuit contains relays to translate certain status signals from the Burroughs printer into logic levels. It also contains a Schmidt trigger which responds to the BFIN signal (Burroughs Finished).

\subsection{Burroughs Event Signals (Dwg. 3.5.8)}

This circuit contains relays which are used to convert certain signals from the Burroughs printer into logic levels. Signals produced are EOES (End of Endorse Signal) and DEDS (Document Edge Signal). 
The circults shown in these drawings are used to implement the printing of the audit trail on the Burroughs printer. The Burroughs logic was modified to allow for a two-pass operation in which the audit trail would be printed first on the reverse side of the check and then the amount would be printed on the face. Throughout the discussion, the term MICR refers to the printing of the amount.

\subsection{Drawing 3.5.9}

The modified Burroughs printer logic includes a flipflop whose state indicates whether the audit trail or the amount is being printed. This is designated the audit trail flipflop and the signal from it is designated ATF. This signal is filtered to remove transients (Dwg. 3.5.15) and the filtered signal is FATF. As shown in Drawing 3.5.9, the FATF signal is inverted to obtain a signal which is on when the amount is to be printed. This signal is combined with the MCRPR (MICR Print) signal to produce the AMPR(Amount Print-) signal. This is applied to an OR gate, together with the NATPR-(New Aud1t Trail Print-) signal, the output being the BRPR (Burroughs Print) signal. The Inverse of this signal is also produced.

Drawing 3.5.9 also shows a clrcult for lnhibiting the printing of the amount or the audit trail.

\subsection{Drawing 3.5.10}

This drawing shows the circults which produce the IAT(Inhibit Printing of Audit Trail-) signal, the GAT-(Gated Audit Tra1l-) signal, and the NATPR (New Audit Trail Print) signal. The GAT- signal is produced by combining the FATF (Filtered Audit Trail Flipflop), PRCY (Print Cycle), and MCROK (MICR OK) signals. The GAT- signal is applied to a one-shot, the output of which is combined with the ATEN(Audit Trail Enable) signal to produce the IAT- (Inhibit Audit Trail Print) signal. The GAT- signal is also inverted and combined with the ATPR (Audit Trall Print) signal to produce the NATPR- (New Audit Trail Print) signal. The inverse of this signal is also produced.

\subsection{Drawing 3.5.11}

This drawing shows the one-shot used to produce the NFAT (New Finished Audit Trail) signal and its inverse. The inverse (NFAT-) is applied to an OR gate with the RPBR(Repeat Burroughs-) signal and the output is inverted to 
produce the RMAC-(Reset Memory Address Counter-) signal.

An OR gate is used to produce the LDBR (Load Burroughs) signal. Its inputs are the LDAT-(Load Audit Trail-) and LDMCR-(Load MICR) signals.

An AND gate is used to produce the LPMCR-(Load and Print MICR-) signal. Its inputs are the LDMCR (Load MICR) and MCRPR (MICR Print) signals.

\subsection{Drawing 3.5.12}

This drawing shows a set of one-shots used when a printing operation is to be repeated manually. The Repeat signal triggers a 150 millisecond one-shot. The inverted output of this one-shot is used to trigger a $70 \mathrm{millisecond} \mathrm{one-}$ shot which then triggers a 2 microsecond one-shot to produce the RPTPL (Repeat Pulse) signal.

The CKM and MCM signals are applied to an OR gate, the output of which is gated with the output of the first of the above one-shots. The output of this gate is inverted to produce the RPBR-(Repeat Burroughs-) signal. It is also applied to an OR gate together with the LDBR (Load Burroughs) signal, and the output of this gate is inverted to produce the CLRBR (Clear Burroughs) signal.

\subsection{Drawing 3.5.13}

This drawing shows the circuit used to couple a signal from the Burroughs logic into the RPD logic which indicates when the equipment has been placed in manual operation. An optical coupling circuit is used. The output is the MAN(Manual-) signal.

\subsection{Drawing 3.5 .14}

This drawing shows the circuit used to develop the manually produced MSTRS-(Master Reset) signal. A normally closed pusihbutton is used to trigger a one-shot for this purpose.

\subsection{Drawing 3.5 .15}

The Burroughs printer logic contains a flipflop whose state indicates whether the audit trail or the amount is being printed. The output of this flipflop is the ATF (Audit Trail Flipflop) signal. The output of this flipflop is optically coupled into the RPD logic to provide isolation and to provide a time constant long enough to block any transients which might occur on this signal. The output 
is the FATF (Filtered Audit Trail Flipflop) signal. 
Modification of the Model S102/S103 Electronic Proof Encoder

\section{Table of Contents}

1.0 Introduction

2.0 Functional Description

3.0 Mechanical Modifications

4.0 Electronic Modifications

5.0 Functional Description of Electronic Modification

\section{References}

(a) Final Report "Modification of the Model S102/S103 Electronic Proof Encoder", Contract No. 2-35905. Prepared by Burroughs Corporation, Federal and Special Systems Group. June 15, 1973.

(b) Burroughs Manuals for the Model S102/S103 Electronic Proof Encoder 
The check processing element of the RPD is a modified Burroughs Corporation S102/S103 MICR Proof Encoder. Th1s device is used by banks to MICR Proof Encode and endorse checks. In the RPD application the keyboard input and tape printer have been removed and certain other electronic and mechanical modifications have been made to enable the device to perform the required functions and interface with other components within the system.

\subsection{FUNCTIONAI DESCRIPTION}

The Burroughs S102/S103 MICR Encoder has been modified by the Burroughs Corporation to allow checks to receive, in addition to normal data, a MICR encoded AUDIT TRAIL message on the reverse side. The system operates as follows: Each check is inserted upside down with the reverse side facing the operator. It then recelves a MICR encoded AUDIT TRAIL message on the reverse side. Next, the check is diverted out of the main stream, by a solenold actuated gate, inverted to the right-side-up orientation, and reinserted into the existing check feed station. The check is then processed thru the $\mathrm{S102/S103}$ in a normal manner to recelve the MICR encoded CHECK AMOUNT message on the face of the check, the endorser message on the reverse slde of the check, and is then stacked in the receiver pocket.

The electronic control system of the S102/S103 MICR Proof Encoder has been modified to allow it to communicate with, and operate as an integral part of the RPD. All message data to be MICR encoded on the check is electronically entered into the S102/S103 control system directly. The keyboard normally supplied with the S102/S103 has been removed. The S102/S103 system provides check position signals and functional status signals to the RPD control circuitry.

\subsection{MECHANICAL MODIFICATIONS}

Mechanical modifications required to provide the second pass of the document through the MICR printer are described in Ref. (1). In addition the following S102/S103 mechanical modifications have been made:

(1) Removed and relocated the left front leg as shown In Drawing No. 7.1.17.

(2) Removed and discarded keyboard pan.

(3) Provided cutouts in existing Formica top to accommodate check path clearances. See Drawings Nos 7.1.4 and 7.1.5.

(4) Provide a two plece plastic cover for the check path. See Drawing No. 7.1.6 and 7.1.7. 
The modifications to the electronics of the S102/S103 required to provide control signals, encoding and interface are specified in the Burroughs Corporation final report (Ref. (a)). The S102/S103 maintenance manual (Ref. (b)) describes the circuitry before modification and should be used in conjunction with Ref. (a) for a complete understanding of the S102/S103.

\subsection{FUNCTIONAL DESCRIPTION OF ELECTRONIC MODIFICATION}

(1) An additional photocell circuit has been added to the transport path following the ENDORSER to indicate completion of full cycle of encoding.

(2) The existing ENDORSER photocell has been moved upstream. This change is necessary due to the interference which the new AUDIT TRAIL transport diverter gate creates with the present endorser photocell location.

(3) Logic has been modified so that the normal MICR encoding function includes two passes of the document. The first, to start after AUDIT TRAIL DATA or associated inhibit signal are stored, is for encoding the AUDIT TRAIL with 36 characters printed on a check which has been inserted by the operator upside down and with the rear of the check facing the operator. The second pass to start after AMOUNT data or associated inhibit signal are stored will be a normal MICR mode encoding of the amount, the check having been returned to its normal position by the new AUDIT TRAIL transport path. Elther of the two encodes may be inhibited by signal from the Remittance Processing System. Such inhibit signals prevent printing but will not affect transport operation.

(4) Logic has been modified so that the AUDIT TRAIL PRINT commences at about an inch following the document edge (which is indicated by the first DOCEDGE signal).

(5) Logic has been modified to receive the AUDIT TRAIL as a 6-bit serial input per character for a total of 36 characters. Additionally, a NO AT MSG. control signal is received and included in the logic. This latter signal when received will cause the AUDIT TRAIL cycle to continue but the AUDIT TRAIL print is inhibited.

(6) Logic has been modified so that the first DOCEDGE signal will result in activation of the solenoid operated transport diverter gate which, in turn, will route the check through the new audit trail transport path. The first DOCEDGE signal will also inhibit the endorser function by inhibiting 120 
the endorser photocell output.

(7) NO JAM signal is generated by the S102/S103. This is already designed into the RPD logic as a result of a time out count which is generated from the DOCEDGE signals and Complete Cycle (new Photocell following ENDORSER) signals generated by the S102/S103.

(8) Logic has been modified so that the 2nd DOCEDGE signal will reactivate the ENDORSER photocell function and will deactivate the solenoid operated transport diverter gate, thereby allowing the document to travel the normal path on the second pass including the ENDORSER and terminating in the receiving pocket.

(9) Logic has been modified so that the second document edge signal will cause the normal AMOUNT MICR encoding function to print unless inhibited. The logic is modified to accept AMOUNT MICR Mode data in a six bit serial fashion. Additionally a control signal is received called NO MICR MSG. This control signal when present will not affect the transport cycle but will inhibit the AMOUNT Print. The second DOCEDGE signal will create a MICR PASS signal to be sent to the RPD.

(10) Logic has been modified to produce a Complete Cycle signal based on receipt of the new photocell output following the ENDORSER function. The second DOCEDGE signal will also initiate a time out cycle which will create a JAM signal unless the Complete Cycle signal is generated within the time out period.

(11) Logic has been modified to allow external input and storage of the AUDIT TRAIL data, 36 characters wide. This input replaced the normal keyboard input and therefore keyboard constrained logic was appropriately modified.

(12) Logic has been modified to allow external input and storage of up to 10 characters of AMOUNT data to be encoded on the second pass of the check through the encoding station. This input is to replace the normal keyboard input and associated keyboard constrained logic has been modified.

(13) Logic has been modified to exclude all tape printer logic functions where such would have constrained the AUDIT TRAIL register or its controls, the AMOUNT register or its controls, the Data Compare Logic, the Transport Logic, the Encoder Logic, or the Endorse Logic. 
(14) Provision for input and output interconnection with the Remittance Processing System has been made using those interconnecting cables and connectors made available due to the removal of the keyboard unit and the tape printer unit.

(15) Logic associated with the Skew 2 photocell has been modified to allow automatic override during the first AUDIT TRAIL pass and until first DOCEDGE for special document conditions such as a corner-off condition.

(16) The following additional signals are provided to the Remittance Processing Device: Ribbon Tension, and Ribbon Out, Interlock Open, and Data Recelved. The Data Received signal will acknowledge receipt of the data (or inhibits) for each pass.

(17) A modification was required to permit operation of the S102/S103 when the original keyboard was removed. The S103 contains a serial number switch which the S102 does not have. When the keyboard is removed, the line to the input of this switch must be grounded.

(18) A modification was made to indicate the mode of operation (Audit Trail Path or Amount Encode Path) via a flipflop activated by the Skew 1 photocell output. The mode flipflop output is sent to RPD as Audit Trail Flip-Flop (ATF).

(19) The RPD sends to the S102/S103 an Audit Trail consisting of 36 characters as previously indicated. Their pointer symbol is coded as 11 (blank) and their space by a 15 code. The S102/S103 will ignore these when they occur and fill in the necessary symbols and spaces via patchboard.

(20) Prior to sending the Audit Trail data, the RPD sends a clear signal which the S102/S103 will pass on to the system unaltered. Following the Audit Trail the S102/S103 generates a Debit signal.

(21) Following the Audit Trail encoding completion, the RPD loads AMOUNT data. There is no Clear signal prior to this data. A Debit code is generated by the RPD following the AMOUNT data.

(22) The Skew 2 signal is provided with a manual override to handle corner out conditions. This allows the operator to examine a check that will not transport through the Encoder section because of an apparent skew condition. If the operator finds the corner torn or otherwise out she may manually override the skew circuit and allow the check to pass through. 
(23) Only one Encoder Ribbon signal is sent to RPD. It represents either Ribbon Out or Ribbon Broken (two series switches) to the RPD. This is RADR signal.

(24) No Data Received signal is generated to the RPS from the S102/S103. This is not a required signal. 


\section{Section V \\ Modification of the Model 37 \\ Teletype for the RPD \\ Table of Contents}

1.0 General

1.1 Requirements

1.2 Type and Location of Modification

1.2.1 Ribbon

1.2.2 Stunt Box

1.2.2.1 Shift Out

1.2.2.2 VT Set and VT Clear

1.2.2.3 HT Set and HT Clear

1.2.2.4 New Line

2.0 Alarms Provided

2.1 Paper Low

2.2 Paper Out

2.3 Physical End-of-Line

2.4 Power Loss

3.0 Wiring Changes 
List of Tables

Table 1. Signal Description -- Teletype Connector P 303

Table 2. RPD to Teletype Cable 
The Teletype Corporation's Model 37 Receive Only Teletype unit was chosen for application in the Remittance Processing System. Its most important feature for this application was its impact printing utilizing a two color ribbon. Other required features were obtained by making small modifications to the unit.

\subsection{Requirements}

Minor modifications have been made to the Teletype Mode1 37 Receive Only unit in order to meet the following requirements:

(1) The need for certain alarm circuits required by remittance processing procedures;

(2) Compatibility with, and inter-connection to, the RPD control Electronics;

(3) To provide additional time to complete operations taking more than one character time without sending NUL characters;

(4) To provide omission of certain information on the first copy of the register form although other copies of the form must contain the information.

\subsection{Type and Location of Modifications}

The following modifications were incorporated to fulfill the requirements listed in paragraph 1.1 .

\subsubsection{Ribbon}

The standard black/red Teletype ribbon was replaced by a special ribbon consisting of a black inked upper half and an uninked lower half with a barrier between the areas to prevent the black ink from bleeding into the uninked area. This permits the printing on the second copies without the information appearing on the first copy.

\subsubsection{Stunt Box}

The stunt box wiring and mechanism were modified to provide desired responses, inhibit undesirable responses, and provide signals to the RPD control electronics.

\subsubsection{Shift Out}

The Shift Out function was disabled with a clip on stunt box slot number 30 . 


\subsubsection{VT Set and VC Clear}

Clips were placed in stunt box slots 39 and 40 to disable the Vertical Tab Set and Vertical Tab Clear functions.

\subsubsection{HT Set and HT Clear}

The Horizontal Tab Set and Horizontal Tab Clear functions were disabled by placing clips in slots 15 and 16 of the stunt box. Before disabling, the horizontal tab stops were set at positions 12, 14, $21,24,29,31,36,37,50$, and 55 on the Teletype scale. The tabs can be cleared and reset by the message generator after removing the disabling clips. The clips should be replaced after resetting the horizontal tabs to prevent accidental changes. The left margin stop is set at position 9 on the Teletype scale.

\subsubsection{New Line}

The New Iine Function, which is a combination of carriage return and line feed, was enabled by removing the stunt box clip from slot number 4 .

\subsection{ALARMS PROVIDED}

\subsection{Paper Low}

A sensor switch was mounted on the paper feed bin so that a -12.5 volt signal is maintained as long as there is paper in the bin. When all paper has been removed from the bin, the switch contacts open and the Paper Low alarm is generated and displayed on the Control Panel. When this occurs, there is sufficient paper still available to complete any batch of documents that has been started.

\subsection{Paper Out}

The Paper Out switch located on the Teletype unit provides a zero volt signal until a paper out condition occurs. Its state then changes to +12.5 volts and a paper out alarm is generated and displayed on the Control Panel. The paper out condition will occur when printing is begun on the last sheet or if a torn section of paper passes the paper out switch. 


\subsection{Physical End-of-Line}

In order to insure against failure to obtain a carriage return after printing a register line, a physical end-ofline switch was mounted on the motor base of the Teletype unit. This may be set anywhere along the line so that a maximum normal writing line will be accepted, but a line exceeding the selected length will cause an alarm. The switch is wired to provide -12.5 volt signal as long as the line length is less than the present alarm length.

\subsection{Power Loss}

A loss of power on the AC line, or a +12.5 volt power supply fallure will cause the DTR (data terminal ready) signal to change to zero volts. This interrupts printing by the Teletype unit.

\subsection{WIRING CHANGES}

Wiring for the Paper Out alarm and for the modifled DTR (data terminal ready) is shown on drawing number 5.4. Drawing number 5.5 shows wiring for the Paper Low and End-of-Line switches as well as the modified stunt box switching and modified ribbon shift wiring. Power supply circuits are shown on drawing numbers 5.1 and 5.2 .

All wiring changes have been made at plugs and jacks to preserve the separability of the unit's different sections. Tables 1 and 2 provide cabling information and signal descriptions. 
Table 1

Teletype Connector P303 (RS-232)

\begin{tabular}{|c|c|}
\hline $\begin{array}{l}\text { Pin 非 on } \\
\text { P303 }\end{array}$ & Signal Description \\
\hline 1 & Protective Ground \\
\hline 2 & $\begin{array}{l}\text { Paper Out Alarm: Paper OK }=\text { OV; } \\
\text { Paper Out }=+12.5 \text { volts }\end{array}$ \\
\hline 3 & Received Data \\
\hline 7 & Signal Ground \\
\hline 12 & $\begin{array}{l}\text { Paper Low Alarm: Paper } \mathrm{OK}=-12.5 \text { volts; } \\
\text { Paper Low }=0 \text { volts }\end{array}$ \\
\hline 14 & $\begin{array}{l}\text { End-of-Line: Not EOL }=-12.5 \text { volts; } \\
\text { EOL }=0 \text { volts }\end{array}$ \\
\hline 20 & $\begin{array}{l}\text { Data Terminal Ready: }+12.5 \text { volts if Power } \\
\text { Supply is on; OV if off }\end{array}$ \\
\hline 21 & $\begin{array}{l}\text { New Line: Voltage Changes From }-12.5 \text { to } \\
\text { OV for one Character Time }\end{array}$ \\
\hline 23 & $\begin{array}{l}\text { Form Feed: Voltage Changes From }-12.5 \text { to } \\
\text { oV for one Character Time }\end{array}$ \\
\hline 24 & $\begin{array}{l}\text { VT. HT., and FF: Voltage Changes }-12.5 \text { to } \\
0 \text { Volts for Duration of Function }\end{array}$ \\
\hline
\end{tabular}


Table 2

RPD To Teletype Cable

\begin{tabular}{|c|c|c|c|}
\hline $\begin{array}{l}\text { RPD } \\
24 \text { PIN } \\
\text { ELCO }\end{array}$ & Signal & Color & $\begin{array}{l}\text { DB 25-S } \\
\text { Teletype } \\
\text { RS-232 } \\
\text { J303 }\end{array}$ \\
\hline A & Data In (To Teletype) & $\mathrm{R}$ & 3 \\
\hline$E$ & NL & $\mathrm{R} / \mathrm{W}$ & 21 \\
\hline $\mathbf{F}$ & FF & $\mathrm{R} / \mathrm{BK}$ & 23 \\
\hline $\mathrm{H}$ & $\mathrm{Tab}$ & 0 & 24 \\
\hline $\mathrm{J}$ & EOL & 0/BK & 14 \\
\hline $\mathrm{K}$ & Paper Out & GN & 2 \\
\hline L & Paper Low & $G / W$ & 12 \\
\hline M & DTR & $\mathrm{G} / \mathrm{BK}$ & 20 \\
\hline $\mathrm{X}$ & Signal GND & $\mathrm{BL} / \mathrm{W}$ & 7 \\
\hline W & Protective GND & DK & 1 \\
\hline
\end{tabular}


Section VI

\section{The RPD Control Panel}

\section{Table of Contents}

1.0 General

1.1 Location and Description

1.2 Functions

2.0 Physical

2.1 Front Panel

2.1.1 Layout

2.1.2 Component Description

2.2 The Box

2.2.1 Layout

2.2.2 Components

3.0 Electrical

3.1 Wiring

3.1.1 Wiring Diagrams

3.1.2 Cables

3.2 Terminal Board Circuits

4.0 Displays and Signals

4.1 Warnings

4.1.1 Display Lights

4.1.2 The Audible Alarm

4.1.3 The Test Switches 
4.2 Control Signals

4.2.1 General

4.2.2 Manual Switch

4.2.3 Selector Switch

4.2.4 Operations Switches 
2.1 Front of Control Panel

2.2 Layout of Display Messages

2.3 Buzzer Mounting Bracket

2.4 Box Layout

3.I Sht A Wiring Diagram

3.1 Sht B Wiring Diagram

3.1 Sht C Wiring Diagram

3.1 Sht D Wiring Diagram

3.1 Sht E Wiring Diagram

3.1 Sht $F$ Wiring Diagram

3.2 Display Signal Cable Wiring

3. 3 Components on $\mathrm{TB}-1 \& \mathrm{~TB}-2$

3.4 Components on $\mathrm{TB}-3$

3.5 Components on $\mathrm{TB}-4 \& \mathrm{~TB}-5$

4.1 Display Unit - Warning Circuits

4.2 Results of Pressing Test Switches

4.3 Control Signals

\section{Table}

3.1 Summary of Display Box Wiring 


\subsection{GENERAL}

\subsection{Location and Description}

The RPD Control Panel is physically configured as an eightinch cubical box with the lower rear portion cut out to clear the check track which runs beneath it. Its front panel contains the display lights and switches required to perform its functions. The front panel and the aluminum box are black anodized except for the inscriptions on the front panel which are clear. It is located at the right rear of the keyboard shelf in front of the operator.

\subsection{Functions}

The RPD Control Panel through its message display unit, lights, and buzzer, informs the operator when a malfunction occurs. It also alerts the operator if a document required for the operation has not been provided.

The switches on the front panel provide means for carrying out manually-controlled printing operations or for cancelling print commands. These capabilities are required for unusual occurrences such as recovery from malfunctions.

\subsection{PHYSICAL}

\subsection{Front Pane1}

\subsubsection{Layout}

The layout of the front panel is shown in Figure 2.1. The informational displays are grouped on the left side of the panel. The upper right of the panel contains the controls. Two sixteen-pin terminal boards are mounted behind the panel on four screws in the lower right of the panel.

\subsubsection{Component Description}

The display unit in the upper left corner is an IEE Series 360 12-message display unit with messages arranged as in Figure 2.2. This arrangement permits a three-line message with an appropriate color background to be displayed. The background colors used in this device are red and yellow. The drive circuits for the display are located in the box, and all leads from the display go to a 56-pin connector used to interconnect the front panel components with those attached to the box. 


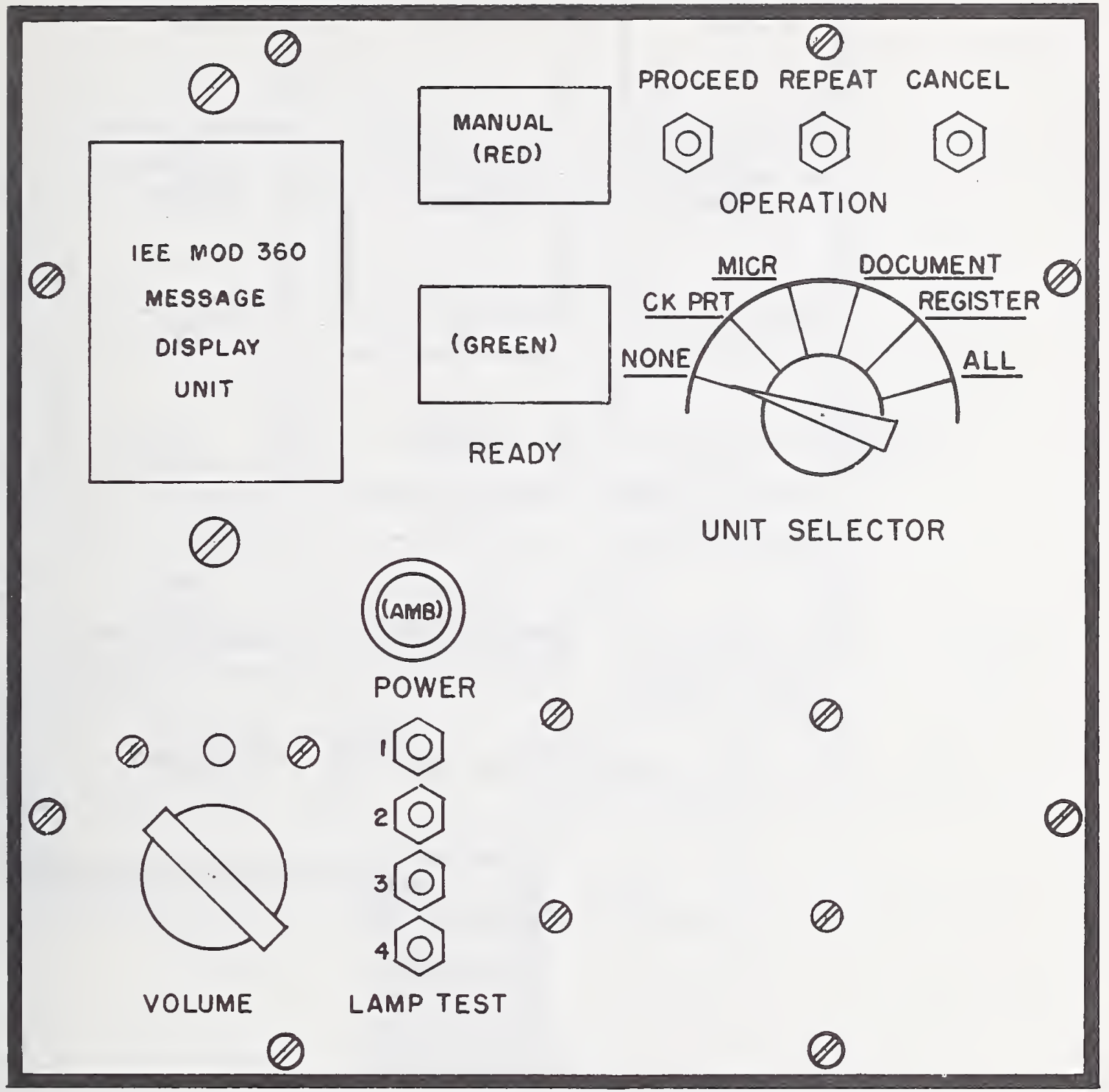

FIG. 2.I - FRONT OF CONTROL PANEL 

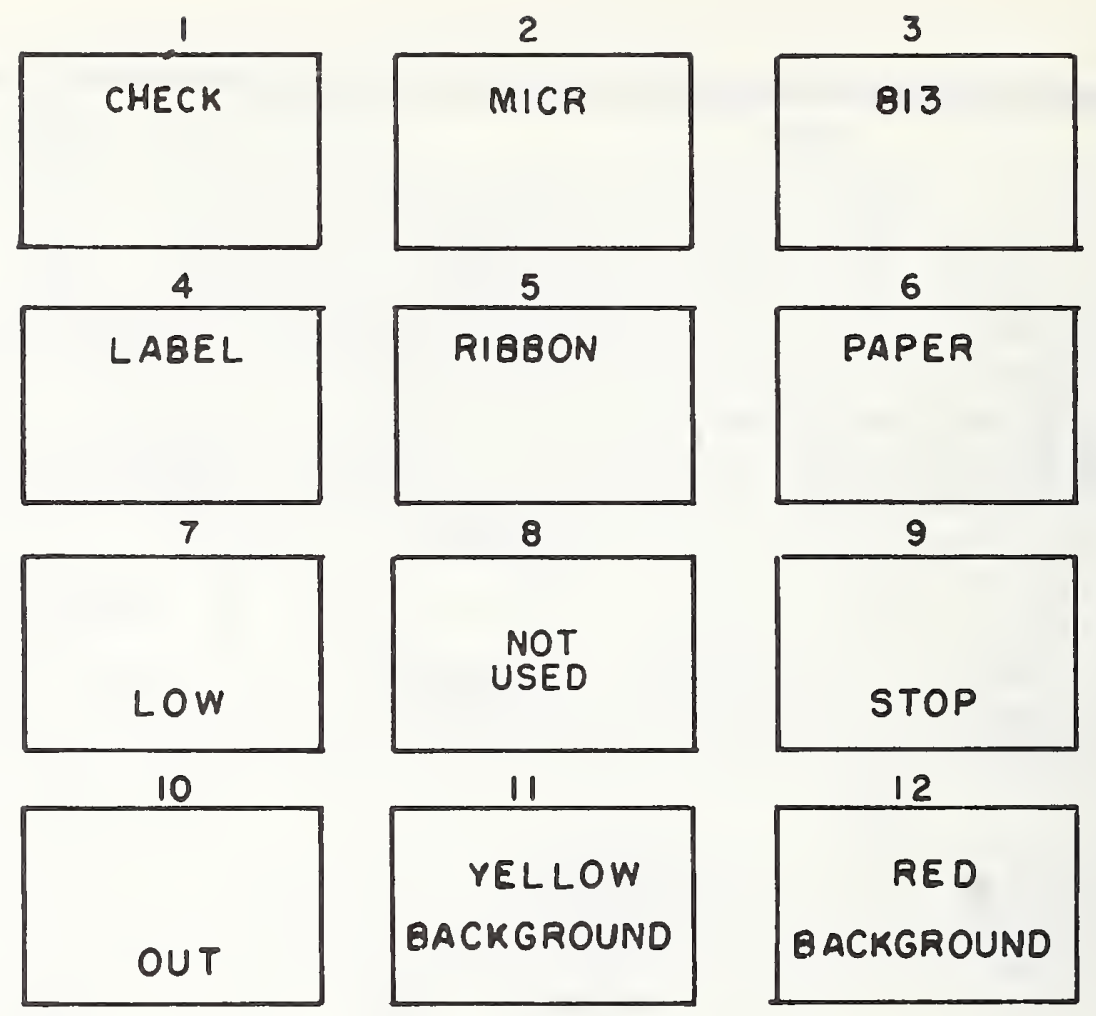

8
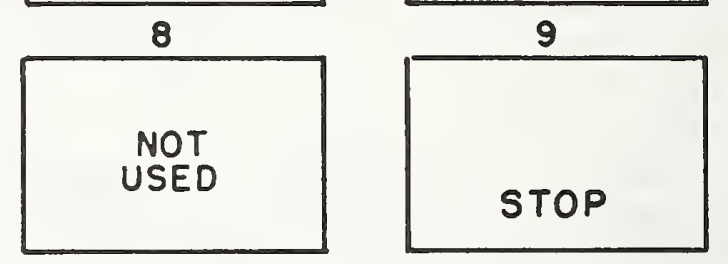

11

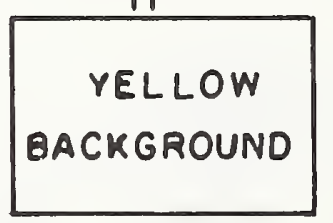

12

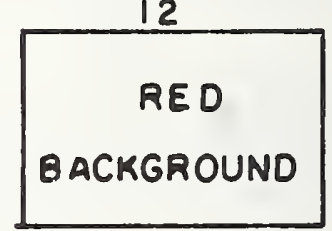

A. LAYOUT OF DISPLAY MESSAGES

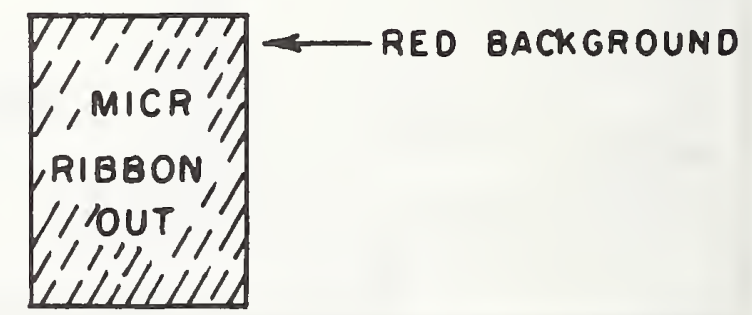

\author{
B. TYPICAL MESSAGE
}

FIG. 2.2 - LAYOUT OF DISPLAY MESSAGES 


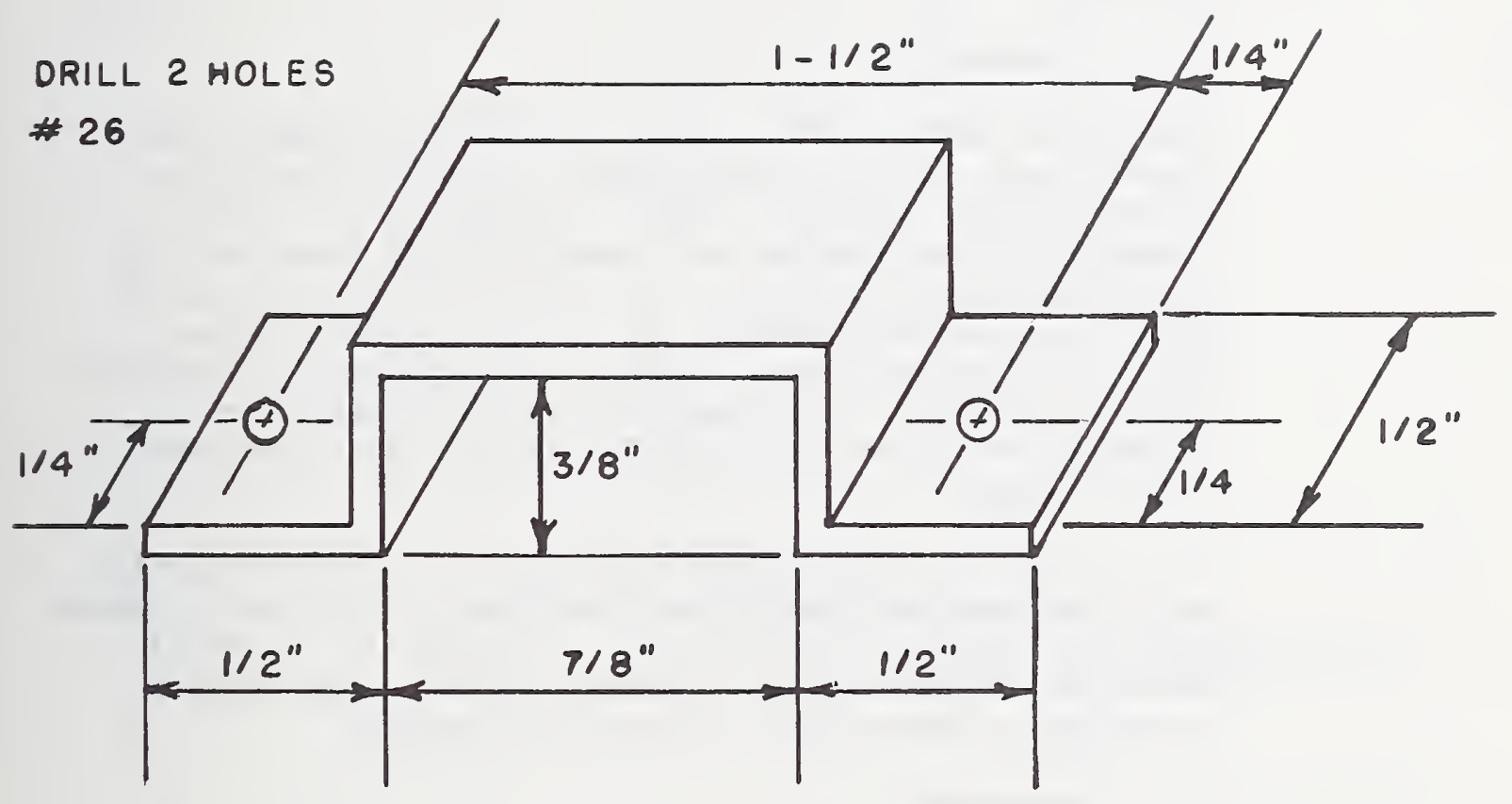

MAT'L: I/I6"AL

FIG. 2.3 - BUZZER MOUNTING BRACKET 
The Ready light is a four-lamp display light with a plain green top. The Power light is a Dialco pilot lamp with an amber jewel.

The Manual Switch is a DPDT switch with a four-lamp illuminated red persh button with the legend "MANUAL." The other push-button'switches are of a normally-open miniature type. The Selector switch is a single-section six-position wafer switch.

The audible alarm is a miniature electronic buzzer. Its volume is controlled by a miniature 50-ohm potentiometer.

\subsection{The Box}

\subsubsection{Layout}

The box is made in three pieces as shown in Figure 2.4. These pleces are held together by metal screws. The back cover is removable to permit changing of the display unit lamps without removing the front panel from the box. The back cover is held on the box by two screws inserted into a pair of 8-32 tapped holes in the box flange. All other lamps are replaceable from the front of the front panel. The front panel is attached to the box with (8) 3/8-inch 非 flat-head sheet metal screws.

The 6.3 VAC filament transformer and a 4-terminal barrier strip are mounted inside the lower back of the box. Inside the right hand side of the box are mounted three 60 pin terminal strips mounted on screws, using a nut on each screw behind the strip to serve as standoffs.

\subsubsection{Components}

A 56-pin receptacle is used for signal connections between the RPD electronics and the Control Panel. A three-pin miniature Twist-Lok male chassis mounted plug is used to connect to the 115 VAC line cord. This supplies current to a 6.3 VAC filament transformer whose output leads are connected to a 4-terminal barrier strip. The other electronics components are mounted on three 60-pin terminal strips.

\subsection{ELECTRICAL}

\subsection{Wiring}




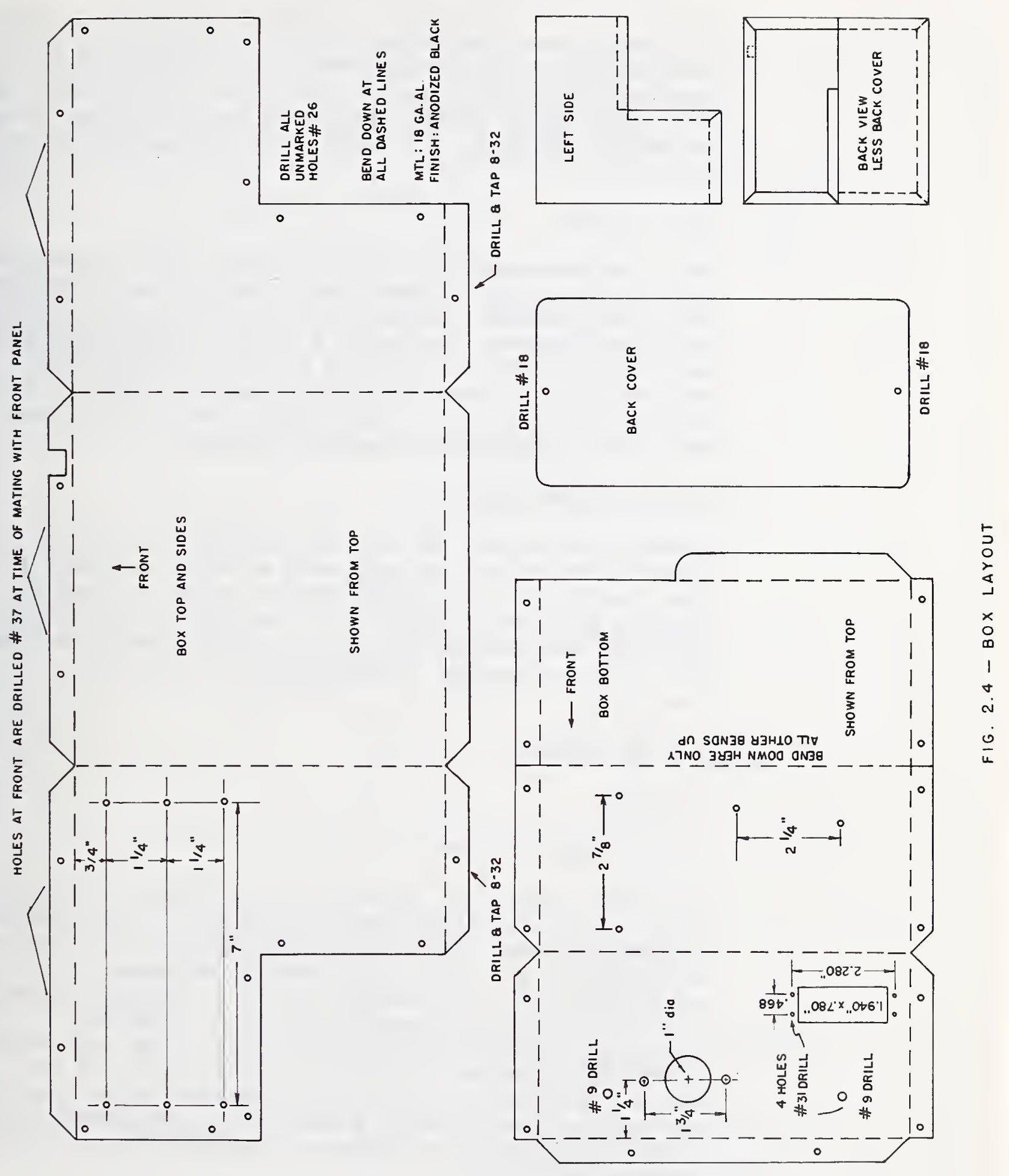




\subsubsection{Wiring Diagrams}

Wiring among the components is shown in Figure 3.1. Sheets A through $C$ show box wiring; sheets $D$ through $F$ show front panel wiring. All wiring between the two sub-units is through $\mathrm{P}-103 / \mathrm{J}-103$. Most of the wiring uses "rainbow" RMA color-coded strip cable. The legend for the wiring diagrams is shown on Sheet $C$. A summary of the wiring appears in Table 3.1.

\subsubsection{Cables}

The input power cable is a standard light-duty three-wire cable with a parallel blade plug on one end, and a miniature Twist-Lok connector with central ground on the other end. The signal cable to the RPD is a 10-ft. cable terminated at each end by a 56-pin plug wired as shown in Figure 3.2. Both cables come through a hole in the table and plug into the bottom of the box. Ground connections to the chassis were made by removing the anodized surface.

\subsection{Terminal Board Circuits}

Terminal boards TB-1 and TB-2 are alike and seven SCR circuits are mounted on each board as shown in Figure 3.3 . The circuitry for this is on terminal board TB-3 and is arranged as shown in Figure 3.4 Isolation diodes for the four test switches are mounted on terminal boards TB-4 and $T B-5$. The arrangement of these diodes is shown on Figure 3.5.

\subsection{DISPLAYS AND SIGNALS}

\subsection{Warnings}

\subsubsection{Display Lights}

All lights except the Manual Light are driven in the same manner. The circuit is shown in Figure 4.1. One side of the lamp is connected to the common 6.3 VAC bus, while the other side is grounded through one of two paths. The operational path goes through a silicon controlled rectifier to signal ground. The gate is driven from the RPD electronics through a $10 \mathrm{kilohm}$ resistor. The gate is held at signal ground for the OFF conditions, and at +5 VDC for the ON condition. Since AC is applied to the lamp, the SCR regains control within a cycle after the control signal returns to ground. The lamps are energized only on the positive half-cycle. 

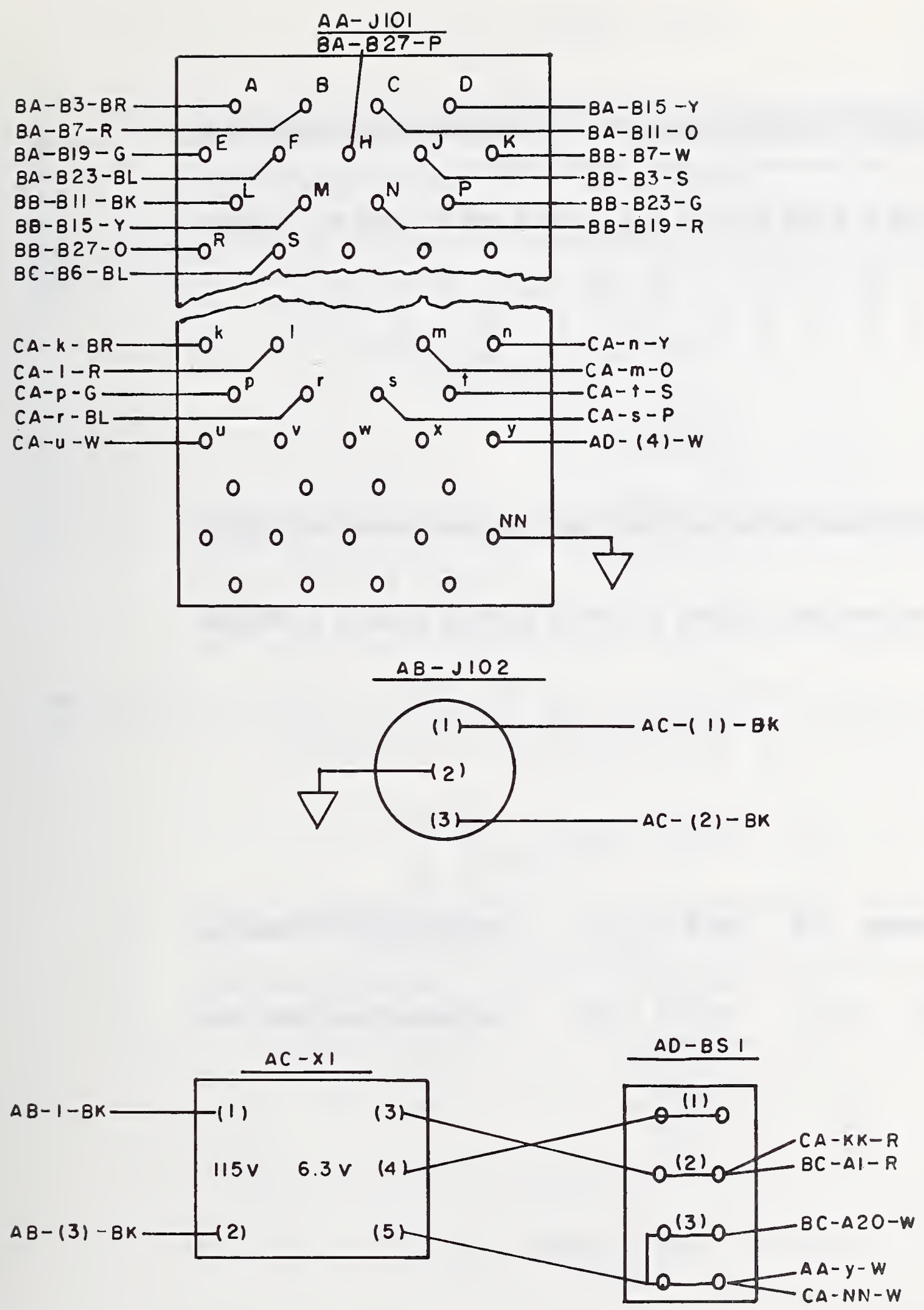

FIG 3.1 - WIRING DIAGRAM, SHEET A 


$$
\begin{aligned}
& 3 \\
& 1 \\
& N \\
& 8 \\
& 1 \\
& 0 \\
& 0
\end{aligned}
$$

$$
B A-T B I
$$

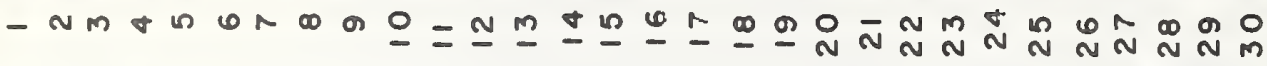

\begin{tabular}{|c|c|c|c|c|c|c|c|c|c|c|c|c|c|c|}
\hline $\begin{array}{ll}\frac{\pi}{0} & \frac{\pi}{0} \\
0 & 0 \\
1 & 1 \\
8 & 8 \\
1 & 1 \\
0 & 4\end{array}$ & $\begin{array}{l}a \\
1 \\
0 \\
1 \\
0 \\
u\end{array}$ & $\begin{array}{c}a \\
1 \\
0 \\
1 \\
a \\
a\end{array}$ & $\begin{array}{l}0 \\
1 \\
1 \\
0 \\
0\end{array}$ & $\begin{array}{l}0 \\
1 \\
1 \\
4 \\
4\end{array}$ & & & $\begin{array}{l}2 \\
\vdots \\
1 \\
1 \\
0\end{array}$ & & $\begin{array}{l}0 \\
1 \\
w \\
1 \\
5\end{array}$ & & 8 & & & \\
\hline
\end{tabular}

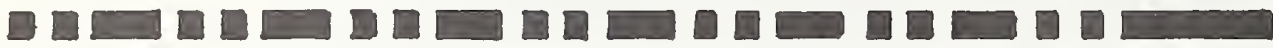

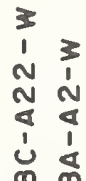

$$
B B-T B 2
$$

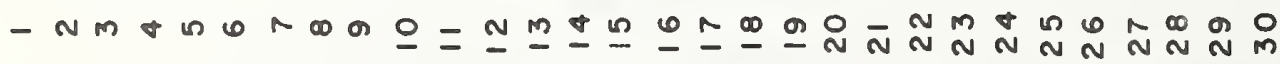

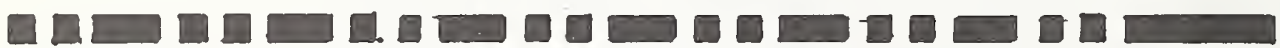

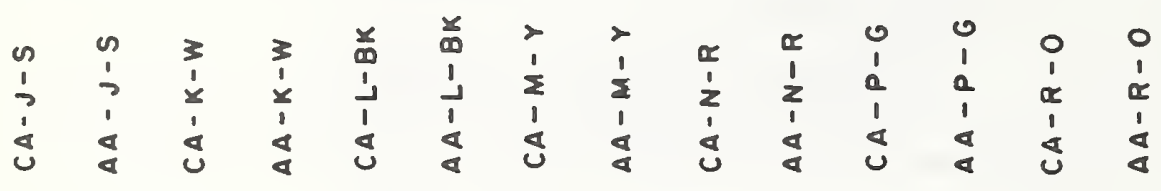

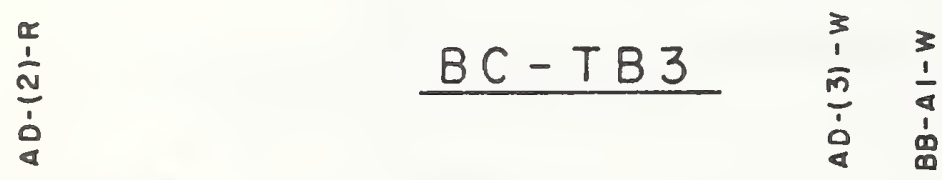

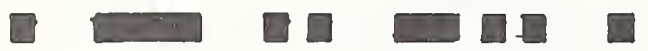

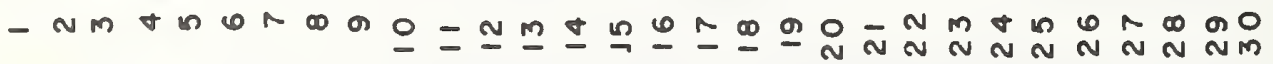

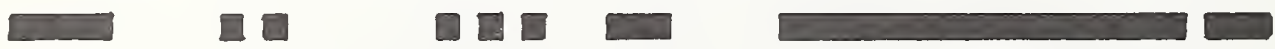

$\begin{array}{lll}\overrightarrow{1} & \overrightarrow{0} & \overrightarrow{0} \\ 0 & 1 & 1 \\ 1 & 0 & 0 \\ 1 & 1 & 1 \\ \vdots & 0 & 0\end{array}$

$\frac{1}{1}$

FIG. 3.1 - WIRING DIAGRAM, SHEET B 


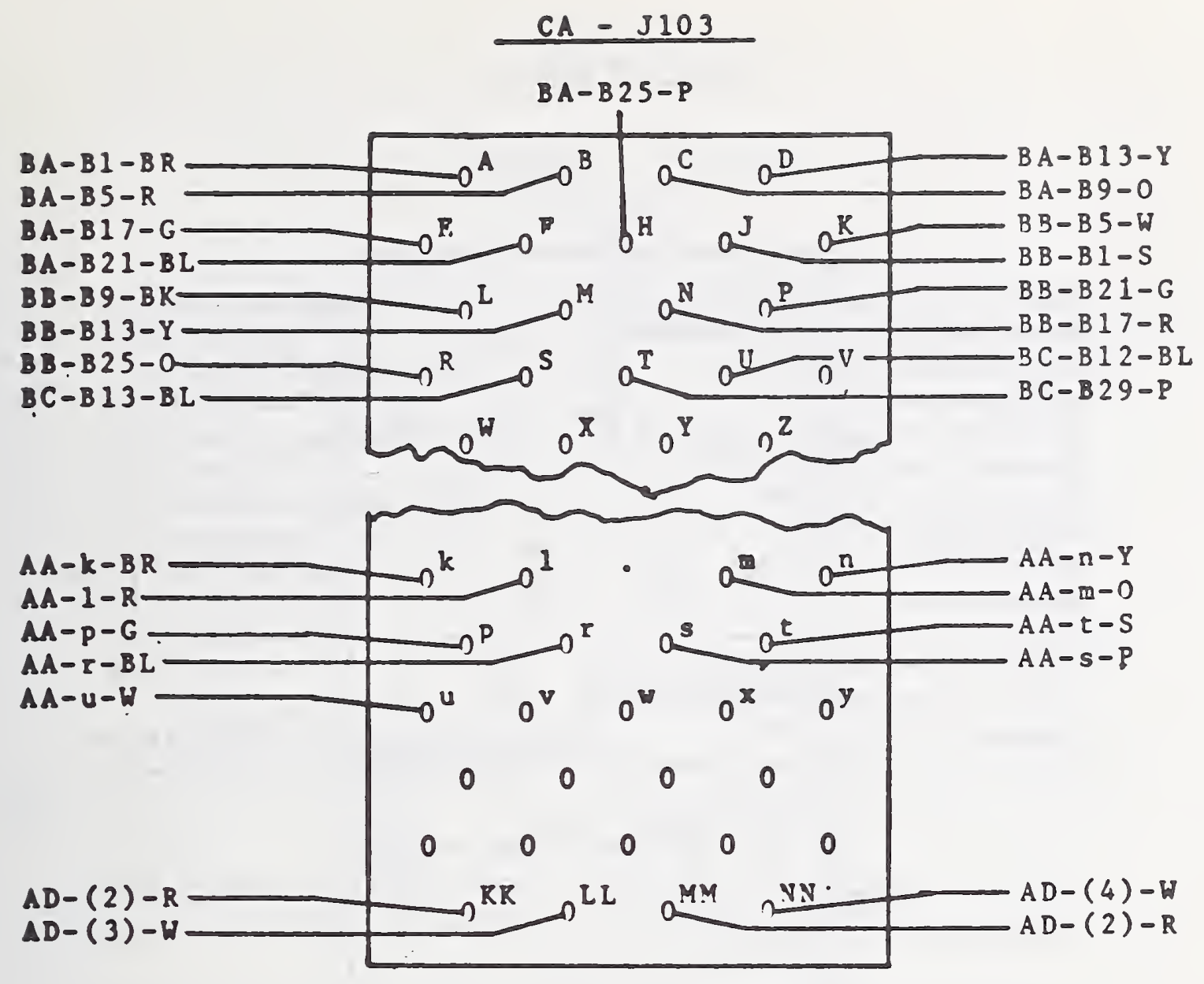

\section{IEGEND}

$X X-Y Y-Z Z \quad()=$ Not Marked

\begin{tabular}{|c|c|}
\hline BR-brown & $\overline{B L}-b 1$ ue \\
\hline $\mathbb{R}$-red & $P$ - Durple \\
\hline 0 -orange & $-8 r a y$ \\
\hline Y -yel1ow & $H$-white \\
\hline G-green & $\mathrm{BR}-\mathrm{b} \mathbf{1} \mathrm{ack}$ \\
\hline
\end{tabular}

PIG. 3.1--WIRING DIAGRAM, SHEET C 

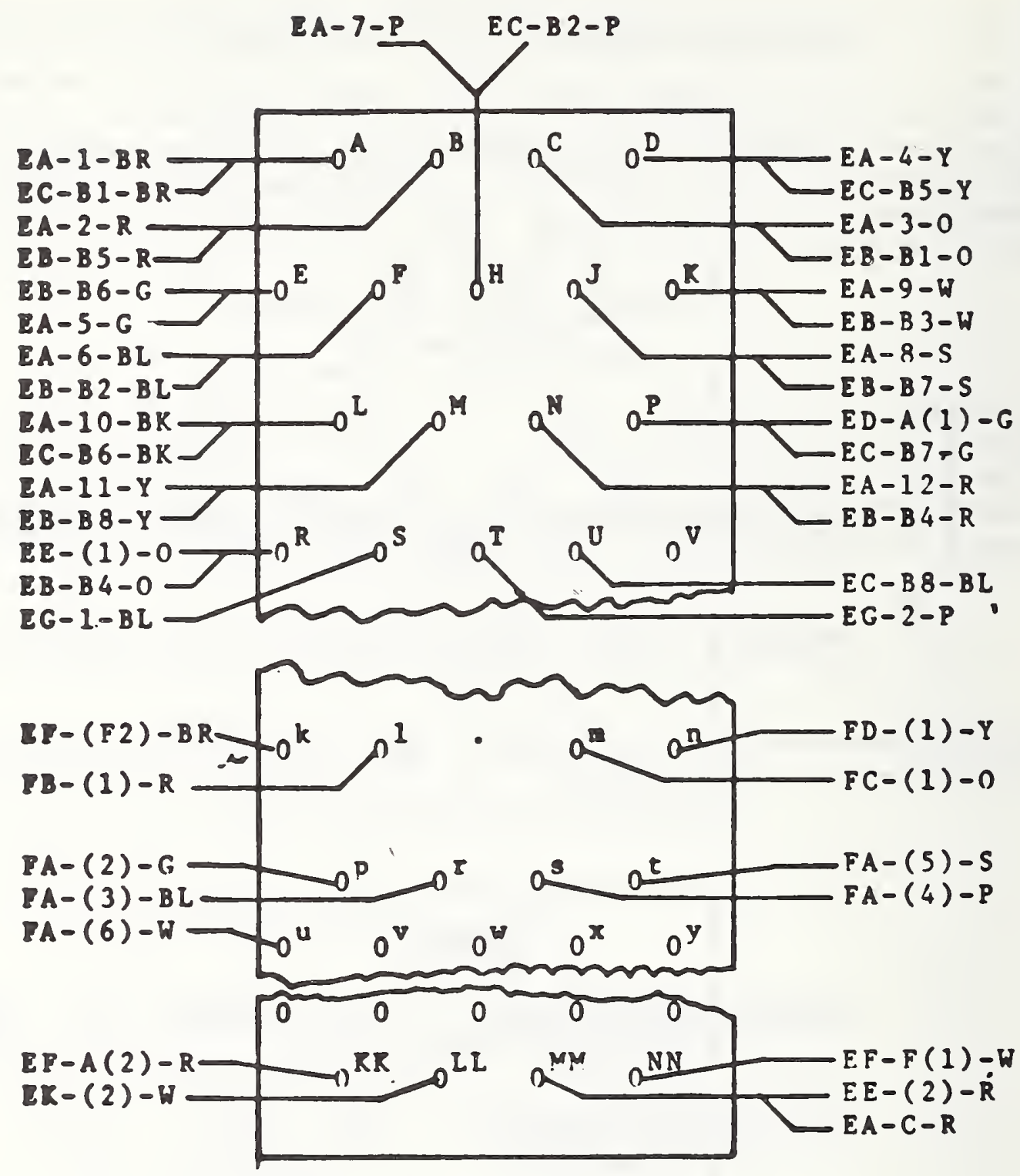

PIG. 3.1--WIRING DIAGRAM, SHEET D 

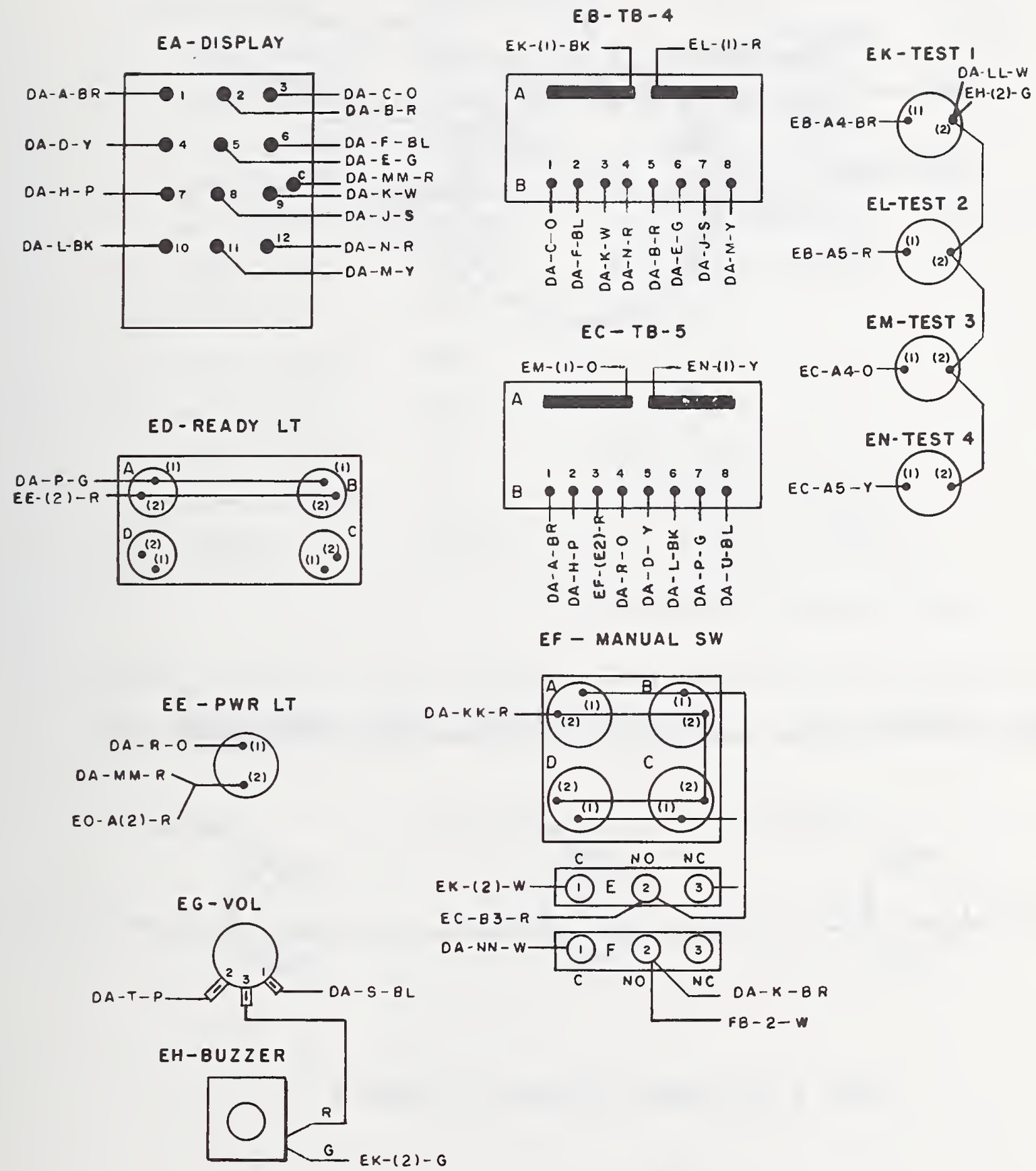

FIG. 3.1 - WIRING DIAGRAM, SHEET E 

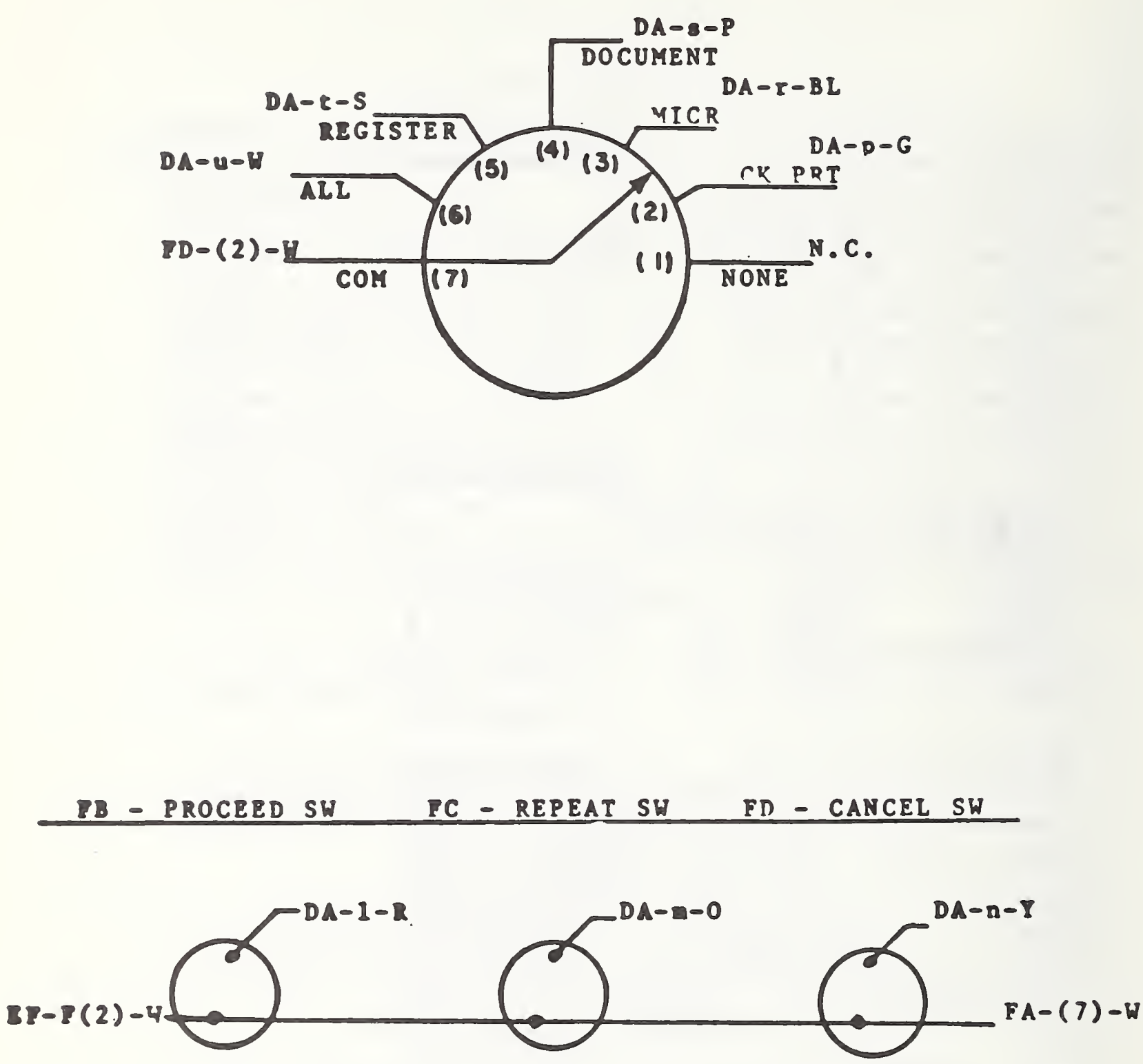

FI6. 3.1-OHIRING DIAGRAM, SHEET F 
PIN TO DISPLAY

A CHECK IIGHT R

B MICR LIGHT $O$

C 813 LIGHT G

D LABE LIGHT BL

E RIBBON LISHT R/BK

$P$ PAPER IIGH' $\mathrm{O}$ T BR

H LOW LIGHT G/BR

J JAM LIGHT

$\pi$ STOP LIGHT

L OUT LIGHT

M YEL. BKGRND

N RED BKGPND

$P$ READY LIGHT

R POWER LIGHT

S BUZZER $W / R$

$R / O$

$R / r_{\text {, }}$

$\mathrm{R} / \mathrm{BL}$

$\mathrm{G} / \mathrm{W}$

$G / n$ $k$ MANUAL SWITCH $\mathrm{P} / \mathrm{W} / \mathrm{BK}$

1 PROCEED SWITCH $R / W$

m REPEAT SWITCH $R / B K$

n CANCFI SWITCH W/R/BR

P CHF.CK PRINTER BK

I MICR PRINTER BK/W

S LABFL PRINTER BL/W

t 213 PRINTER. BK/R/W

4 ALL PRINTER.S i

PIN LETR:L

SIGNAL GROUND

G/BK/W

CRASSIS GROUND *

*G WIDF, nUTSIDE CABLE

DISPLAY PLUG: ELCD 56-PIN n0-9016-056-000-720 KFYED 1,1 RPD PLUG: SA:E, KEYED 2,2

FIG. 3.2--DISPLAY SIGNAL CABLE' HIRING 


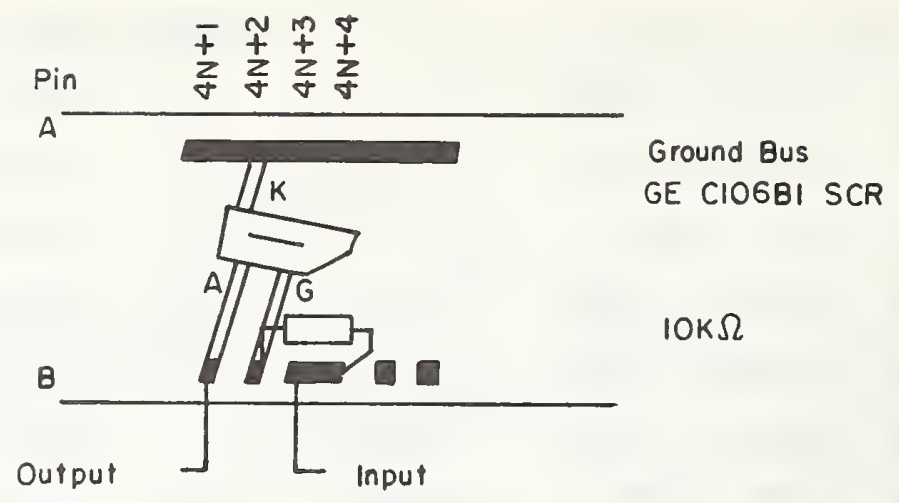

Figure 3.3. Components on $T B-1$ and $T B-2$

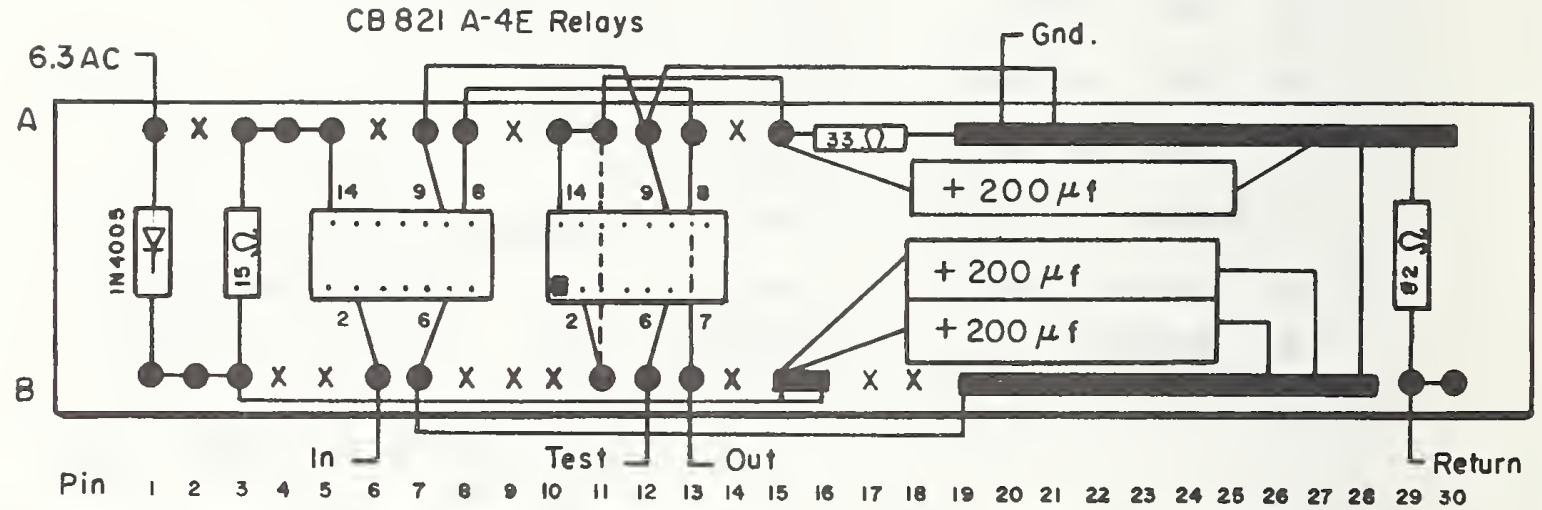

Figure 3.4 Components on $\mathrm{TB}-3$

All diodes of each group of four foces in some direction

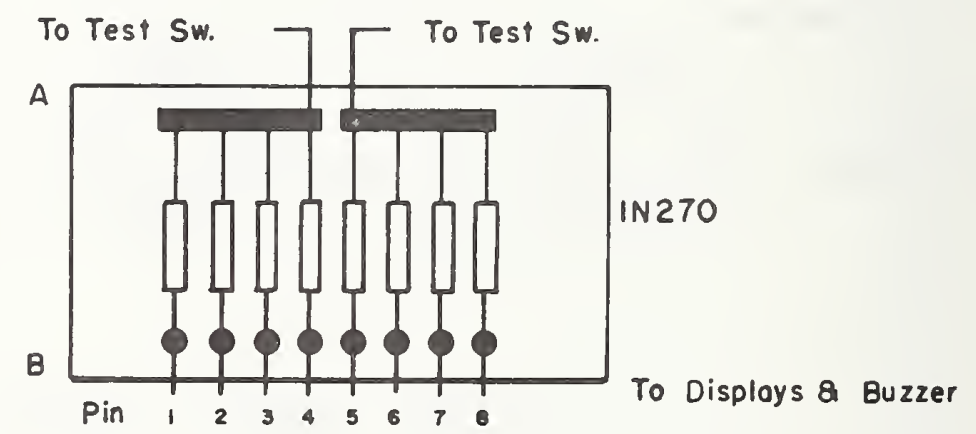

Figure 3.5. Components on TB-4 and TB-5 

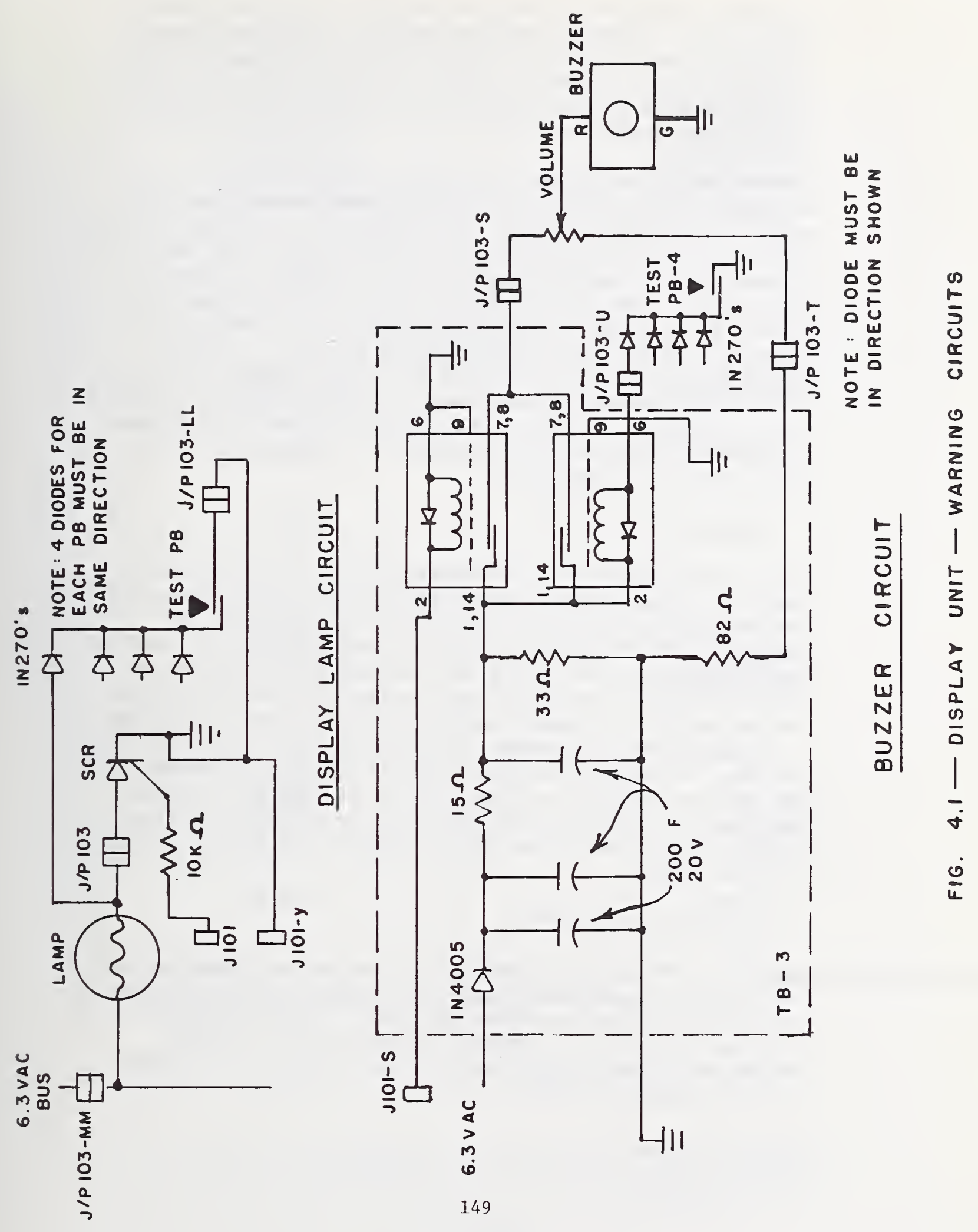
The test path runs through one of four isolation diodes located on a terminal board attached to the front panel, through a test switch to signal ground. The diode limits current flow to one-half cycle of the AC voltage, so that the appearance of the display during test is the same as that during operation.

The Manual Switch is an illuminated pushbutton, two lamps of which are lighted while in the Normal state, and two while in the Manual state. The lamps for the Normal state are wired with a series resistor to provide a low level of illumination to permit reading of the "MANUAL" legend on the button. When in the Manual position, the other two lights are supplied in parallel with 6.3 VAC through one normally open contact of the Manual switch. The Test switch provides an alternative source of current through a diode to the Manual mode lamps. When energized in this way, the Manual mode lamps are not as bright as when they are switch operated, but they are well above the Normal mode level of illumination.

\subsubsection{The Audible Alarm}

The audible alarm is a miniature, DC-operated electronic buzzer. Because it is undesirable to put the elements of the buzzer electronics above ground, this circuit is operated through one of a pair of reed relays. The coil of one relay is driven by the RPD electronics. A positive voltage causes a normally open contact to close, applying the output of a DC power supply to the volume control and a series resistor provided to set a loudness level for the buzzer. The test reed relay coil is connected between the DC power supply and Test Switch 非 4 through a diode. The Test switch, when operated, provides a path to ground, operating the reed relay which connects the Buzzer to the DC power supply.

\subsubsection{The Test Switches}

The Test Switches are provided to assure the operator that the alarms are working. Each of the four switches operates four of the alarms as shown in Figure 4.2. The Test Switches provide an alternate ground circuit through isolation diodes, which prevent cross-feeding of signals during normal operation.

\subsection{Control Signals}

\subsubsection{General}


TEST 1

rerrerrer rrr813rrs IIPAPERIY IIISTOPIY rererrer

TEST 3

CHECK

LOW

retrertr

ITMANUALI

rerrerrer

a

adaaa

aad

a

POWER
TEST 2

VYYYYYYYY YVYMICRVV SYRIBBNNY YYYJAMYVY MYYYYYYY

TEST 4

IABEL

OUT

१९qqवqवqव 999ववqवव g9g9व9g9

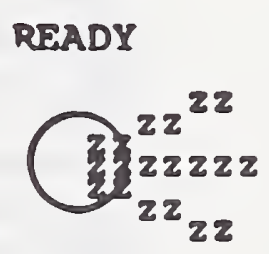

BU\%ZFR

$r$ = red backqround, $y=$ yellow backaround, a = amber background $g$ = green background, $z$ audible tone

FIG. 4.2-RESULTS OF PRESSING TEST SWITCHES 
The manual control switches provide means for operating under abnormal circumstances, such as when recovering from a printer malfunction, or during trouble-shooting. Manual operations consist of placing the Manual Switch in the Manual mode, selecting a printer (or all of them) with the Selector Switch, then depressing one of the three operations switches. Since the message to the RPD is not destroyed until the next message is sent, any operation may be repeated for several printers, or several operations may be carried out for one printer without leaving the Manual mode. At the end of the manual operations, it is necessary to place the Manual Switch in the Normal mode so that the unit responds to new messages. The unit is in the Busy status during manual operations.

All signals to the RPD consist of a switch closure to signal ground as shown in Figure 4.3 .

\subsubsection{Manual Switch}

The Manual Switch is a DPDT lighted push-button switch which provides signal ground level to all the control switches through one of its poles when its normally open contacts are closed. A signal to the RPD is also provided through the same contacts.

\subsubsection{Selector Switch}

This is a six-position rotary switch with its common terminal connected to signal ground through the Manual Switch. During Normal operations it should be set to NONE, which has no output lead. Any single printer or all printers at once may be selected for manual operation. If several, but not all printers are to be operated, they must be set up and operated individually, one after the other, in any order. Each active position of the switch provides a switch closure to signal ground on a separate lead to the RPD electronics.

\subsubsection{Operations Switches}

The operations switches consist of three SPST, normally open miniature pushbutton switches. Only one switch at a time should be operated.

Operating the Proceed Switch causes any selected printer to continue its operation from the point where it may have been interrupted. For some printers, once the actual printing operation has started, it is carried to completion 


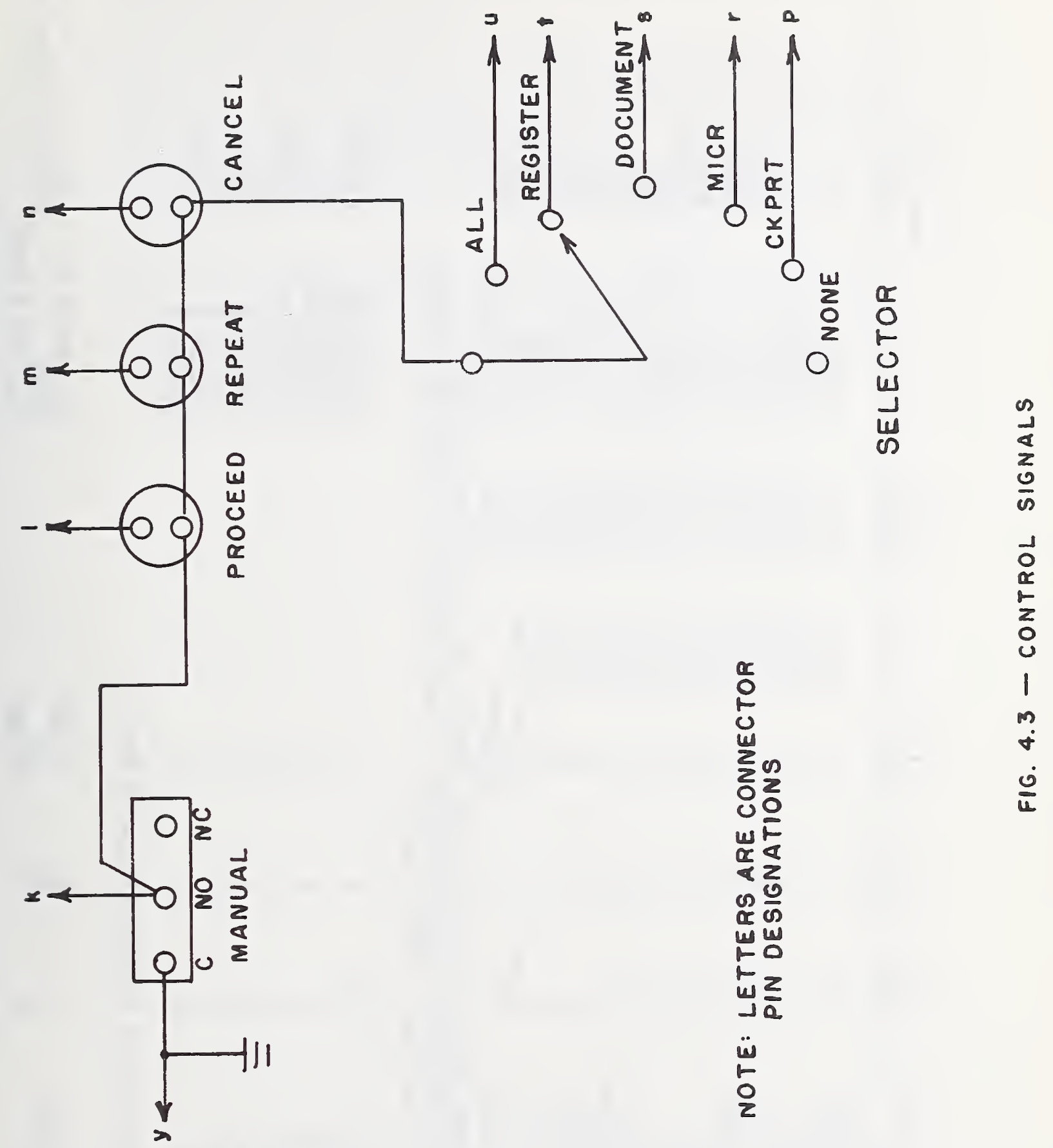




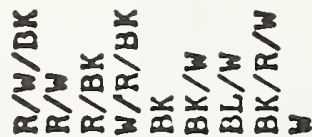

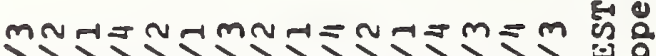

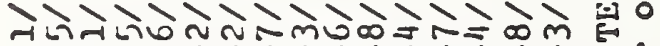

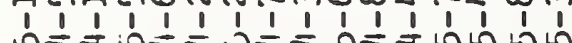

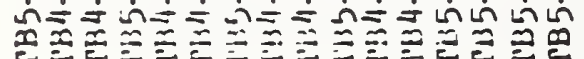

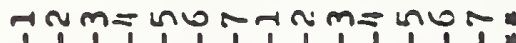

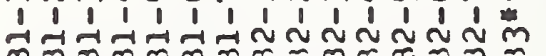

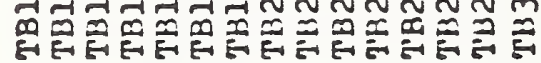

m है

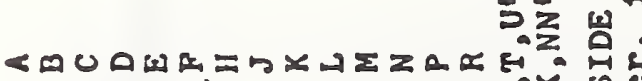

证 远远的舟的的

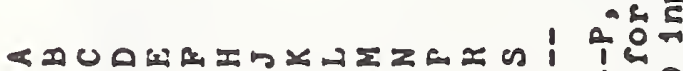
\&in $\rightarrow=0$

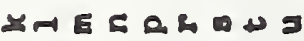

론

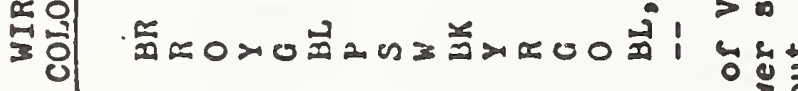
要

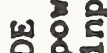
कृ $>0$

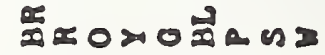

\section{(n)}


despite certain malfunctions. For these printers, the Proceed Switch does not have the usual meaning after the printing cycle has started.

The Repeat Switch causes the complete message to be repeated. If operation is successful, and all alarms are reset at the end of the cycle, return to the Normal mode would cause RPD operations to continue. If operation is not successful, the alarm lights would again come on, and return to the Normal mode would not cause operations to proceed.

The Cancel Switch causes the printer control electronics to step ahead to the condition that would result from a successfully completed printer operation. The printer message is still in the RPD memory, and other operations may be selected after this. 
The RPD Operator's Console

\section{Table of Contents}

1.0 Location and Description

1.1 Floor Plan

1.2 Functional Description

1.2.1 Enclosure

1.2.2 Spatial Arrangement

1.3 Physical Description

1.3.1 Configuration

1.3.2 Decor

2.0 Physical Design

2.1 Structural Framing

2.2 Enclosure Panels

2.3 Work Surface

2.4 Cooling

2.5 Paper Supply Bin

\subsection{Power Distribution}


The work area where the Remittance Processing System is installed is approximately $70 \times 96 \mathrm{feet}$. Approximately one-third of this area is occupied by the RPD and the RPSC. An additional 25 percent of the area is used for six key station terminals (KST) stations, work tables and supervisors' desks. The KST stations are used for original entries into the DDES prior to processing by the RPDs.

The entire area has an elevated flooring system with a carpeted surface. The air conditioning and power distribution are typical of those found in computer installations.

\subsection{Floor Plan}

Each operator's console requires a floor area approximately 12 feet square in order that the operators and supervisors have suitable mobility in the operating environment. This area also provides ample room for maintenance and repair. The ten operators consoles are arranged in two rows. One row contains six consoles and the remaining four are in the second row. The consoles are so arranged that all operators face the same direction. Drawing number 7.0 .1 shows the general arrangement of the system.

\subsection{Functional Description}

\subsubsection{Enclosure}

The general appearance of the console is similar to that of an overgrown office desk. The console surface is 30 inches in height and is 74 inches wide. Its depth ranges from 44 inches on the right hand end to 60 inches on the left hand end. A drop leaf extends the latter dimension to 78 inches.

The three principle functions of the console are to:

(1) Enclose and protect the logical and electrical circuits;

(2) Provide a work surface for the operator to efficiently separate remittances from the document stack, process the remittances, label and restack the documents;

(3) Provide a foundation for several devices which are components of the RPD. 


\subsubsection{Spatial Arrangement}

The RPD console was designed around the Burroughs S102 Electronic Proof Encoder because of its ability to endorse and MICR print on various sizes of checks. A mechanical addition to this equipment provided the capability for another of the required functions of the RPD. Considerable effort was extended in achieving a satisfactory arrangement of the various devices with which the operator must interface.

Various arrangements were sketched, modeled, and operator motions were studied to determine the desirability of the different device groupings. The arrangement chosen for the console has the devices most used by the operator near at hand. The register printer, requiring the least attention from the operator, is placed at the right rear of the table top where it was possible to provide a paper supply bin underneath. The keyboard and display are near the center of the console located at the front and rear respectively with the check track running between them. Also located centrally and between keyboard and display is the RPD Control Panel which recelves frequent operator attention. The Document Labeling Device is located at the front of the console near the operator's left hand. This device is totally contained within the structure of the console and is suspended from a removable portion of the table top. The only visible parts of the labeler are the movable document stops and the document entry slot. A pair of lights are located near the slot to inform the operator when the document may be withdrawn from the device.

The left rear of the surface is unencumbered. A drop-leaf support is located in front of the console at the operator's left side. The right front of the console contains the MICR printer, check endorsing mechanism and stacking area for the endorsed checks. The general arrangement of these devices is shown in Drawing No. 7.0.2.

\subsection{Physical Description}

\subsubsection{Configuration}

The general size of the console, the method of construction, and the weight of the equipment precludes portability. The weight of a complete RPD operator's console, fully equipped is approximately 950 pounds. 
The console shape is similar to that of an L-shaped desk. Drawing Number 7.0.2 shows the basic shape of the console and arrangement of the major components of the RPD.

Drawing Number 7.1 .1 shows the varlous pleces of the surface and gives the basic dimensions of the console. An item not shown in the drawing is a small accessory drawer situated below the surface and to the left of the keyboard pan.

\subsubsection{Decor}

The operators' consoles were finished in different colors for variety. Five basic colors were chosen and each color was used to finish the side panels for two consoles. The electronic proof encoders, which were incorporated into the consoles, were supplied with side panels of dark gray and with framing members of silver aluminum color. The side panels, with the exception of the right-end panel, were unmodified; however, the right-end panels were painted to match the basic color of the console side panels. The console framing was painted to match the framing of the electronic proof encoder. The table top was made of a wood-grained material to match that of the S102 Encoder.

\subsection{PHYSICAL DESIGN}

\subsection{Structural Framing}

The particular style of construction was chosen to be compatible with that used in the Burroughs S102 encoder. Vertical members are square shaped steel tubing and horizontal members are steel angles. The structure was formed by welding these members together, forming a very strong and rigid unit.

The console frame is composed of three major units. One unit was the S102 Encoder frame. The other two framing units were designed and constructed by NBS. Small modifications were made to the S102 in preparation for mating with the NBS framing units. This modification consisted primarily of removing the original keyboard and keyboard pan and the left front leg. The casters were also removed and adjustable helght glides were installed.

The two framing units fabricated by NBS were then clamped to the S102. The frames were then drilled and through bolted, making the console a separable unit for purposes of transportation and moving through doorways. Since each $R P D$ console is prepared in this way, disassembly and reassembly can be done without misalignment problems. 


\subsection{Enclosure Panels}

The enclosing panels are installed between the vertical square tubing with a small amount of clearance between. There is no interconnection between the panels and the vertical frame members. The NBS-fabricated panels are supported at the bottom by two locating pins attached to the lower angle member. At the top, the panels have two expandable fasteners which are inserted into holes in the upper angle member and expanded by a screwdriver.

Exceptions to this method of fastening exists on the two panels located on the left end of the console. For these panels, lever-operated catches are installed, allowing quick removal without tools for access to the document labeler and RPD logic circults. A small door is installed in the rear of the console for access to power switching.

\subsection{Work Surface}

The work surface or tabletop was designed to be compatible with and to make maximum use of the tabletop. furnished with the electronic proof encoder. A wood-grained plastic covering was cemented to a $1^{\prime \prime}$ chip board base. Portions were cut out as necessary to provide openings for equipment, cables, and paper forms. The tabletop was made in several pieces for conventence in handling and ease in construction. Attachment to the structural framing was accomplished with machine screws through the upper angles of the frame into threaded inserts installed in the tabletop.

The drop-leaf surface was prepared simflarly on a plywood base and attached to a special support angle by a plano hinge. A folding support is used underneath to allow the drop leaf to hang in a vertical position.

\subsection{Cooling}

An exhaust fan was installed to prevent the occurrence of localized hot spots. This fan was installed in the rear panel alongside the RPD logic chassis, and was mounted at a height fust above the logic chips. A continuous crossflow of air was thus provided to maintain approximately the same temperature throughout the volume occupied by the RPD logic circuitry.

\subsection{Paper Supply Bin}

A multiple copy, fan-folded, pin-feed form is used by the Register Printer. A supply of this form paper must be 
available at all times; therefore, it was necessary to provide a paper supply bin to meet this requirement. A commercial paper bin was obtained and to satisfy requirements of the system, a paper-low switch was installed in the bottom. This switch is lever-actuated and is held in the operated position by the weight of the paper forms. When this weight is removed, the switch returns to its unoperated state and a signal is sent to the RPD Control Panel which displays an 813 Paper-Low signal to the operator.

\subsection{POWER DISTRIBUTION}

Each RPD station is supplied with three power lines. One power line is a special circuit and is associated with the central computing system. This source is used to supply power to the keyboard and display equipment provided by IRS. The other two power lines provide power for the remainder of the RPD station.

These three power inputs are carried to each RPD via underfloor cabling to receptacle boxes. The RPD units are connected to these by cables terminated with locking type connectors. Inside the RPD there are three multiple receptacle outlet boxes for power distribution to the RPD components. One of these outlet boxes is for the exclusive use of the keyboard and display equipment.

The measured power consumption at an RPD station is less than $1 \mathrm{KVA}$. 
The RPD Document Labeling Device

\section{Table of Contents}

1.0 Introduction

2.0 Requirements

3.0 Mechanical Description of the Document Labeler

3.1 Structural Assembly

3.2 Document Gulding Assembly

3.3 Document Transport Assembly

3.4 Document Stop Assembly

3.5 Reel Assembly

3.6 Label Printer Assembly

3.7 Label Carrier Assembly

3.8 Stamp Assembly

3.9 Label Shear Assembly

4.0 Description of the Document Labeler Logic

4.1 Error Signals

4.2 Phase $\emptyset$, The Idle or Reset State

4.3 Phase 1, Preparation for Printing

4.4 Phase 2, Printing the Label

4.5 Phase 3, Label Handling

4.6 Phase 4, Affixing the Label 
5. 0 Construction

5.1 Mechanical

5.2 Electrical Wiring

5.3 Logic Wiring

6.0 Installation

7.0 Document Labeler Adjustment Procedures

7.1 Heat Adjustment

7.2 Lamp Intensity Adjustments

7.3 Mechanical Adjustments 
1. Placement of 555 in Socket

2. Line Receivers Type 8820 Association of pin numbers with symbols used.

3. Line Drivers Type 8830 Association of pin numbers with symbols used.

\section{List of Tables}

1. Integrated Circuits Used

2. Signal Location Guide

3. Wiring from Sequencer Logic to RPD Logic

4. Wiring from Labeler Sequencer Logic to Electro-Mechanical Devices

5. Wiring from Sequencer Logic to CTS Label Printer

\section{List of Reference Drawings}

Computer Terminal systems, Inc.

CTS Dwg. No.

127882 Rev. A

127885-1 Rev. B

172885-3 Rev. B

127883 Rev. A

126971-M2 Rev. C

126951-M2 Rev. C
Title

Input Data Connector

Schematic, Interface Board

Assembly, Interface Board

Wiring Diagram, Mechanical Assembly

Component Layout, Printer Board

Schematic, Printer Board

164 
The document labeling device was designed and constructed to provide identification on documents for information retrieval purposes. This device produces printed labels and automatically applies the labels to documents properly positioned within the document labeler. Data for printing the label is supplied to the document labeler from the RPD Logic.

\subsection{REQUIREMENTS}

The document labeling device is intended to print a message from the computer on a label and to attach the label to the document associated with a remittance.

The device is capable of printing and attaching labels to 5 documents per minute. An operator is required to properly orient each document, insert it into the document labeler and after the label is attached, remove and stack the document.

Documents to be labeled may vary in size from $3-1 / 2$ by 7 inches to $8-1 / 2$ by 15 inches and may consist of single sheets of thin paper to packets as thick as $1 / 2$ inch. The document may be positioned for label application near one of its 4 edges. The location of the label along the edge is controlled by the operator.

The labels are $1 / 2$ inch wide and four inches long and printed on adhesive coated paper. The adhesive is activated by heat after the printing operation is completed. The label message has 3 groups of numerical digits. The numerical groups are 14 digits, 9 digits, and 10 digits, respectively and are separated by single spaces.

\subsection{MECHANICAL DESCRIPTION OF THE DOCUMENT LABELER}

Major components of the document labeler are mounted on a vertical aluminum plate suspended from a removable portion of the RPD Console tabletop. The document labeler is removable from the RPD Console for maintenance purposes. It is possible to place the document labeler in the RPD Console in two different orientations for operator convenience.

The document labeler is composed of several subsystems performing specific functions controlled logically by the labeler sequencer unit which is also mounted on the main structural plate as an electronic subassembly. 
The major subsystems are:

(1) Structural Assemb1y

(2) Document Guiding Assembly

(3) Document Transport Assembly

(4) Document Stop Assembly

(5) Reel Assembly

(6) Label Printer Assembly

(7) Label Carrier Assembly

(8) Stamp Assembly

(9) Label Shear Assembly

\subsection{Structural Assemb1y}

The structural assembly provides the supporting features to interconnect the other subsystems. It consists primarily of four components. An aluminum angle fastened to the top edge of the main plate is used to suspend the entire document labeling device from the RPD Console tabletop. Another aluminum angle on the opposite side of the main plate is used for supporting motors and electromagnetic solenolds. The main plate carries the remainder of the components. A set of feet is attached to this structure to allow the labeler to be freestanding.

\subsection{Document Guiding Assembly}

The document guiding assembly is a combination of a fixed sheet metal plate (gulde plate) and a shaped sheet metal plece (deflector plate) supported on knife edges. The knife edge suspension serves as a pivotal axis to allow the deflector plate to swing away from the guide plate. Documents of different thicknesses (up to $1 / 2$ inch) are accommodated in this manner. The deflector plate also serves to keep thin documents in close proximity to the guide plate and the vacuum hold-down shoe for minimal disturbance when the stamp applies the label to the document.

\subsection{Document Transport Assembly}

The major components of the document transport assembly are two pulleys, a flat belt and a drive motor. The belt drive motor maintains a constant motion of the flat belt in either direction determined by the position of a toggle switch. When a document is placed in the labeler between the guide 
plate and deflector plate, it rests edgewise upon the drive belt and friction between the document and moving belt carries the document to one of the document stops.

\subsection{Document Stop Assemb1y}

The document stop assembly is composed of two moveable document stops. These stops were made moveable to control location of the labels on the documents. Opto-electronic circuits are associated with each document stop to sense the presence or absence of a properly positioned document. Another opto-electronic sensing device determines whether the document has been inserted deeply enough to contact the drive belt.

\subsection{Reel Assembly}

The reel assembly provides storage for the label material. The reel is fabricated from a hub, two flanges $8^{\prime \prime}$ in diameter and a knob to remove one flange for replacement of label material. The hub is mounted on a shaft with two ball bearings to allow easy motion of the reel. The shaft is supported by a flanged base. The remaining part of the reel assembly is a damper loop and damper spring to reduce overtravel of the reel after label pulling forces have been removed.

\subsection{Label Printer Assembly}

The label printer assembly consists of a commercially supplied strip printer, its associated control electronics and individual power supply. All parts of the label printer assembly are mounted on the main plate of the labeler structure. The printer currently being used was not originally chosen for the Document Labeler. Because the physical design of the Document Labeler was based on the use of another printer it was necessary to purchase an alternate printer which would fit in the avallable space when it was determined that the printer originally chosen was not satisfactory for the job. An off-the-shelf printer which would satisfy both operational and physical requirements was not available; however, a manufacturer was found who could provide, within the alloted time, a printer using its standard components in a special arrangement.

The device prints on a $1 / 2^{\prime \prime}$ wide paper tape with an inked print-wheel and hammer arrangement. The ink is contained in a porous roller which is turned by contact with the print-wheel, assuring a continuous inking of the type faces. A tape feed motor and a photoelectric circuit maintain a sufficient quantity of tape storage in the device to print 
one label. The tape supply is replenished automatically as the stored tape is used by the printing mechanism.

Separating the printed label from the supply tape and applying the label to a document are not done by the printer but are performed by devices external to the printer.

\subsection{Label Carrier Assembly}

The label carrier assembly is primarily a moveable arm, an actuating motor, mechanical devices to control motion of the arm and controls to position the arm at specific places during the sequenced operating phases of the Document Labeler. The label carrier arm is mounted on a horlzontal shaft provided with ball bearings and mounted on the main plate. At the proper time in the operating sequence, the label carrier arm is rotated $90^{\circ}$ about the shaft from its vertical rest position to a horizontal position. After other events it is returned to its rest position. A cam, switches, and logic circuitry govern power and braking control to the actuator motor and bell crank system which provides this motion.

Mounted on the label carrier arm is the vacuum hold-down shoe whlch is heated to actlvate the glue on the label material. The printed label is "captured" by the vacuum hold-down shoe when the electrically operated vacuum solenold valve is opened. Th1s occurs when the label carrier arm is in the horizontal position. After capture, a shearing operation is performed and the label carrier arm is rotated to 1 ts vertical position where under proper conditions, the label is applied to a document.

\subsection{Stamp Assembly}

One of the final actions performed in the labeling operation is to apply the glue coated label to a document. This is done by the stamp assembly which is an electromagnetic solenold mounted at the proper height and provided with a cushioned stamp platen to provide pressure on the document when the solenoid is energized, moving the document into contact with the label. The cushion allows minor varlations in document thickness to be accommodated in the stamping process. The vacuum is released and the stamp platen is returned to its rest position by the platen return spring.

\subsection{Label Shear Assembly}

The label shear assembly is mounted on the label printer. Its primary components are an electromagnetic solenold and 
a small pair of hand shears. A mounting block is used to attach these components to the label printer base plate. The shear is operated by a flexible link attached to the solenoid plunger. After the label has been printed, the solenold is energized and operates the shear, separating the printed label from the label stock. Energy for return of the solenoid plunger and for reopening the shear is provided by the internal spring in the shear mechanism.

This label shear assembly is a newly installed field modification and finished drawings are not available. The included drawings are for an earlier version of the label shear assembly.

\subsection{DESCRIPTION OF THE DOCUMENT LABELER LOGIC}

The label sequencer unit receives data and other signals from the RPD Logic and generates logical functions to control the mechanical functions of the labeler mechanism and to print data on the label. It also provides error signals and completion signals to the RPD Logic. In addition, there are signals provided to the sequencer by mechanical switches and optical circuits within the labeling mechanism itself. The RPD Logic provides the following signals to the Sequencer:

(1) Data for printing the label;

(2) A Print Command (PRCND) signal;

(3) A Label Start (LABST) signal;

(4) A Document Enable (DCEN) signal;

The sequencer provides the RPD Logic with these signals:

(1) Data Print Time (DPT)

(2) Power Out (POWER)

(3) Paper Out (PAPOT)

(4) No Vacuum (NOVC)

Four other signals are received by the Sequencer from the labeling mechanism:

(1) Shoe UP (SHUP)

(2) Shoe Down (SHDN) 
(4) End Detect (ENDT)

Of these, the first two are status indicators for the labeling mechanism while the latter two are used to indicate the presence and proper position of a document in the document labeling device.

In addition to the above signals, the sequencer generates two signals for the printer. The signals generated and transmitted to the printer are the forward/Reverse (FRRV) and the STROBE. The sequencer also gates the data from the RPD Logic into the printer.

From the LABST, SHUP, SHDN, and DCEN signals, the document label sequencer generates the RUN signal and several other intermediate signals, subsequently producing the signals which operate the label printer and the labeling mechanism.

The labeler mechanism operating signals are SMD, BRK, VCSW, STMPD, SHERD, and DCLMP. SMD is the shoe motor (actuator motor) drive signal and controls power to the actuator motor moving the label carrier arm on which is mounted the vacuum hold-down shoe. BRK is the brake signal which stops the actuator motor when the label carrier arm reaches its destination. VCSW provides label holding vacuum to the shoe by opening the vacuum solenoid valve after the printing operation and before the label shearing operation which is controlled by the SHERD (shear document) signal. STMPD is the stamp document signal and controls application of the label to the document. After affixing the label, the DCLMP (document lamp) signal activitates indicator lamps telling the operator to remove the labeled document from the document labeling device.

It is convenient when considering the operating sequence of the document labeler, to describe the operation in phases. The five phases to be described are:

Phase 0 - The Idle or reset state

Phase 1 - Preparation for printing

Phase 2 - Printing the label

Phase 3 - Moving the label to the document station

Phase 4 - Affixing the label

\subsection{Error Signals}

The Print Ready (PRDY) signal from the label printer is sent to the Control Panel through the Sequencer and RPD Logic. 
Absence of this signal turns on the "red" background lamp and the "OUT" lamp in the Control Panel indicating a power out condition.

If the opto-electronic circuit in the printer detects lack of paper tape for a period longer than 2 seconds, a Paper Out signal is generated by the printer, a PAPOT signal is sent to the Control Panel through the Sequencer and the RPD Logic. The Control Panel message is "Label Paper Out."

The labeler's remaining error indication is the NOVC signal. This signal indicates a condition of No Vacuum to the RPD Logic. Although logic circuitry still exists in the equipment, the electropneumatic switching for detecting this condition was not installed and the use of the NOVC signal was abandoned. The redesign of the vacuum hold-down shoe and Improved vacuum system has reduced label drops to a minimum and the use of this signal is no longer considered necessary.

When the RPD Logic recelves either the Power Out or PAPOT signal, operations are inhibited until the situation is corrected. The NOVC signal is wired out since it is no longer used.

\subsection{Phase 0, the Idle or Reset State}

Absence of the document enable signal (DCEN) clamps all circuits into their initial condition. If the shoe (vacuum hold-down shoe) is not in the UP position, absence of DCEN will cause the shoe to rise to the UP position, its proper location in the initial condition. No further action takes place until DCEN goes positive.

\subsection{Phase 1, Preparation for Printing}

The requirement for printing a document message causes DCEN to go positive. The Label Start (LABST) signal from the RPD Logic provides a positive signal to switch the RUN FF into its RUN state. This turns on the LWR FF, starting the shoe motor which keeps running until the shoe down sensor resets the LWR FF. The BRK pulse again stops the shoe, this time in the full down position.

The end of the BRK signal triggers the Data Transfer FF (DTFF) on, and the Data Print Time signal (DPT) is sent to the RPD Logic when the Forward (FWD) and Gated Label Start (GLST) signals are also in the on state. It is during this phase that the label tape is positioned for the printer to begin printing. The tape is positioned by

$$
171
$$


stepping the printer feed motor backwards.

When the RUN FF is $O N$, and the FWD FF is OFF, the STEP and STEP B signals are generated. The STEP signal permits counting of the $A D C N$ pulses in the counter. When $17 \mathrm{ADCN}$ pulses have been counted, the Halt Reverse (HLTR) complement is generated, setting the FWD FF which prevents further backward stepping of the printer motor.

\subsection{Phase 2, Printing the Label}

The message is printed on the label under control of the RPD Logic and the label printer electronics. The printing rate is received from the RPD Logic as the Print Command (PRCND) signal. The label is fed onto the surface of the shoe as the message is printed. When the last character has been printed, the label is not yet in position to be held on the vacuum hold-down shoe and sheared.

\subsection{Phase 3, Label Handling}

After the last character has been printed, the label tape is fed forward for 19 character spaces, the COUNTER is incremented once for each character space. After the 19 th count, a Halt Forward (HLTF) signal is generated turning OFF DTFF which turns the Vacuum (VAC) on, opening the vacuum solenoid valve, so that the label is held to the vacuum hold-down shoe. Setting the VAC FF initiates a delay before the Shear (SHER) pulse is generated which operates the label shear assembly, separating the label from the label printer. The trailing edge of the SHER pulse triggers the Raise (RZE) FF to start the shoe motor and to enable Phase 4. As in Phase 0 , the shoe moves to the UP position. The shoe UP sensor also resets the RZE FF.

\subsection{Phase 4, Affixing the Label}

The end of the Shear Pulse (SHER) enables Phase 4, but nothing can occur until the shoe is in the UP position and the Document Bottom (BTDT) signal and the Document End (ENDT) signal indicate the document is in place. When both conditions are satisfied, a Stamp Pulse (STMPD) is generated which causes the document to be pressed against the label. The end of the Stamp Pulse initiates generation of a Document Finished (DCFIN) Pulse and a Label Finished (LBFN) Pulse. DCFIN is a signal to the RPD Logic and LBFN is used to turn on the Document Lamps (DCLMP) indicating to the operator that the document should be removed from the document labeling device. The document lamps remain lit until the document is removed from the labeler. The vacuum 
solenoid valve is now closed, releasing the label from the shoe. The labeler is now in Phase 0 , and remains in this phase until a DCEN signal is recelved from the RPD Logic.

\subsection{CONSTRUCTION}

\subsection{Mechanical}

Standard types of gear motors and electromagnetic solenoids were chosen for constructing the document labeling device. The layout and basic dimensioning was designed around these components. Mechanical parts for the labeler were machined from standard sizes of aluminum angles, plates, bars and sheets. A few parts were made from stainless steel sheet and a few from brass stock. All parts were designed and machined in NBS facilities.

One major change was made in the original design when it became necessary to choose a more satisfactory printer than had originally been chosen. The impact of this change was minimal considering that mechanically it only required moving the reel assembly downward by two inches and a small movement of the Shear Assembly. The electronic changes involved eliminating a paper loop and a timed paper advance and reversal. Paper advance and reversal were then placed under the control of a counter which provided more precise control of label length and printing location on the label.

A few parts were remanufactured to improve upon the design as construction proceeded. Most notable of these changes were the document stops which went through several stages of experimentation before a satisfactory design was found.

The logic functions for operating the device are located on a separate frame as a subassembly and mounted on the main support plate of the labeler. All electrical connections to the logic is through plug-in connectors so that emergency maintenance can be done on a substitution basis. The printer is also easily replaced, allowing corrective maintenance to be performed on the bench rather than at the operator's console.

\subsection{Electrical Wiring}

The labeler receives power into an electrical switch box provided with an on-off switch and a double receptacle. The $15 \mathrm{~V}$ power supply is supplied with its 115 VAC input from this receptacle. Since the $15 \mathrm{~V}$ power supply is not mounted on the labeler, its output is brought to the labeler by a pair of wires to TB-1. All other wiring is 
done on the labeler terminal board connections and point-topoint techniques. The general wiring schematic is given on Drawing No. 8.3.1.

\subsection{Logic Wiring}

The types of circuits used are listed in Table 1 and, as shown there, are of two types: 15-volt and 5-volt. In order to simplify power wiring, the 15-volt circuits were placed in blocks 1 and 2 and the 5-volt clrcuits in block 3 . The Signetics 555 timer has only 8 pins and is placed in the 14-pin socket as shown in Fig. 1. Logic diagrams using the 555 indicate socket pin numbers, not chip pin numbers. HDRE, HDRF and HDRG are headers made by NBS and their pin numbers are shown on the logic diagrams. On level translator type 666, pins 2,5 and 8 were used as inputs. On level translator type 665 input pins 1, 6 and 11 were used.

Al1 chips have their voltage on pin 14 and ground on pin 7 . The designation $5 \mathrm{PR}$ used on the diagrams means that pin is connected to +5 volts. Note that all level translators type 665 have their pins 13 connected to t5 volts. The correct pin assignment for the DM 8820 and DM 8830 chips to match the symbols used on the logic diagrams are shown in Fig. 2 and Fig. 3. The symbols RES and PR represent pull ups of resistors located on HDRE and their pin assignments are shown on Drawing No. 8.2.16. The connectors are the 28-pin type. They are located in block 1 in socket positions $1,7,13$ and 19. Each pin used has its pin assignment on the logic diagram where it appears.

\subsection{INSTALLATION}

Installation of the document labelers was accompanied by some persistent problems. Prior to shipping and installation, the labelers had been operated and performance checked in the laboratory although thorough mechanical adjustments had not been made. Upon installation of the labelers it was immediately evident that many labels were dropping off of the vacuum holddown shoe before being moved into position for application to the documents. In some cases the label was never "captured" by the vacuum hold-down shoe. In others, the label was being knocked off the shoe either partially or totally by the shear mechanism. These problems were present continually in some labelers, sporadically in others and evidenced themselves in others only when the label paper supply was low and therefore when the paper had excessive curl. These label "drop" or "lost label" problems were reduced by thorough mechanical adjustment and a temporary field modification using a "puffer" to provide a short blast of air over the label at the same time the vacuum solenoid valve 


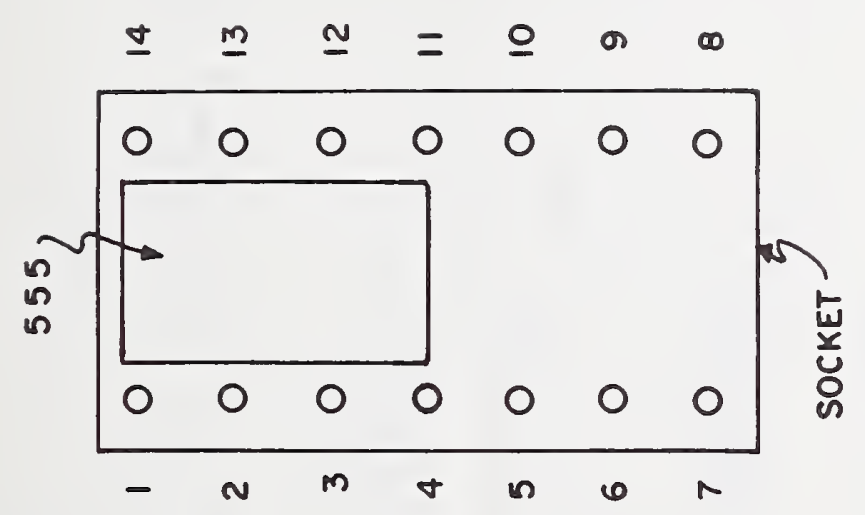

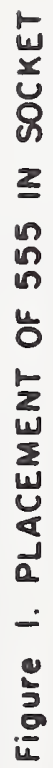

175 

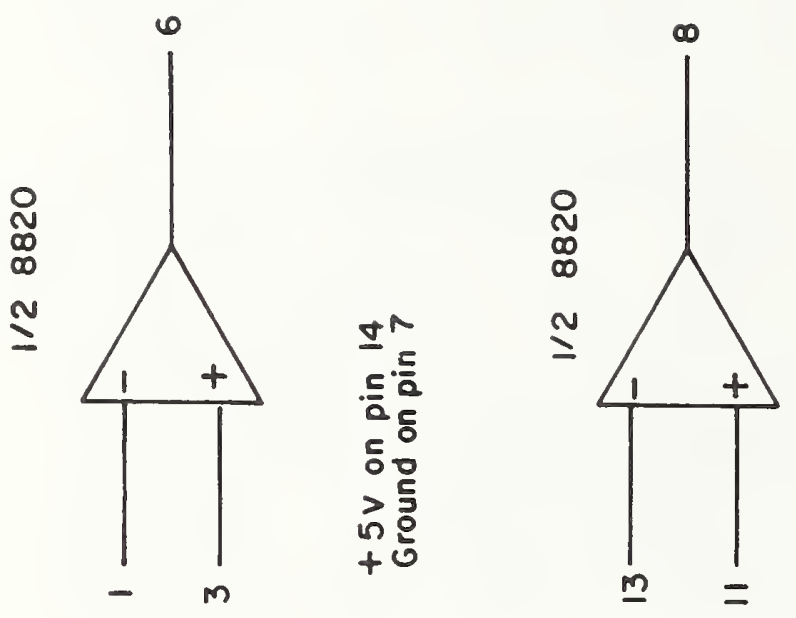

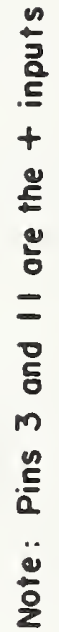

N
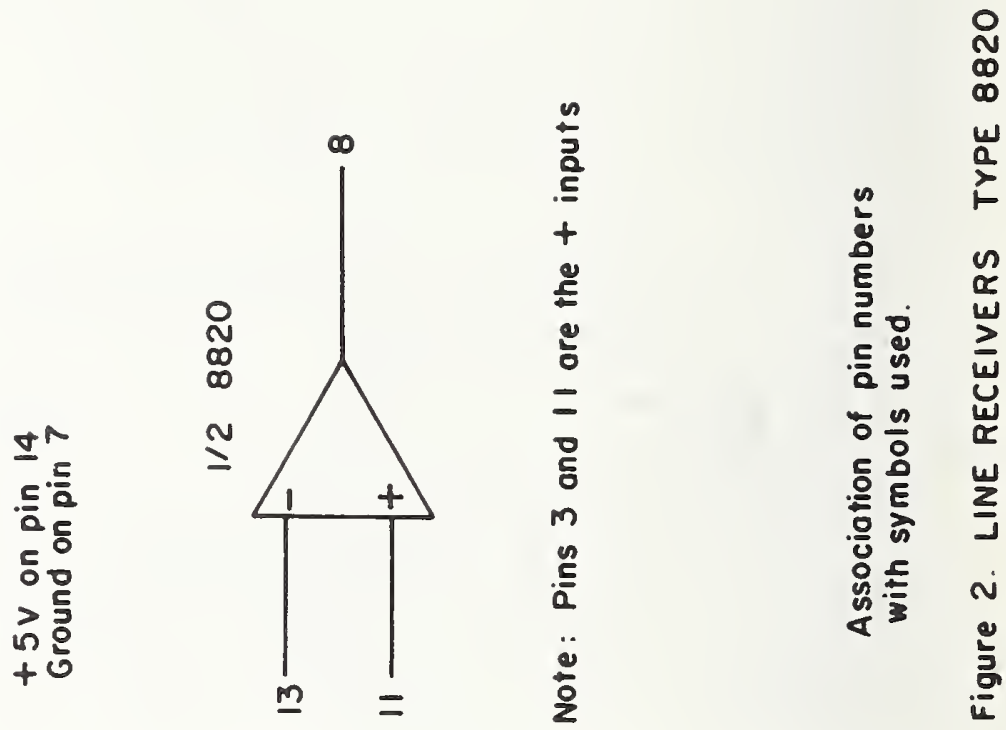

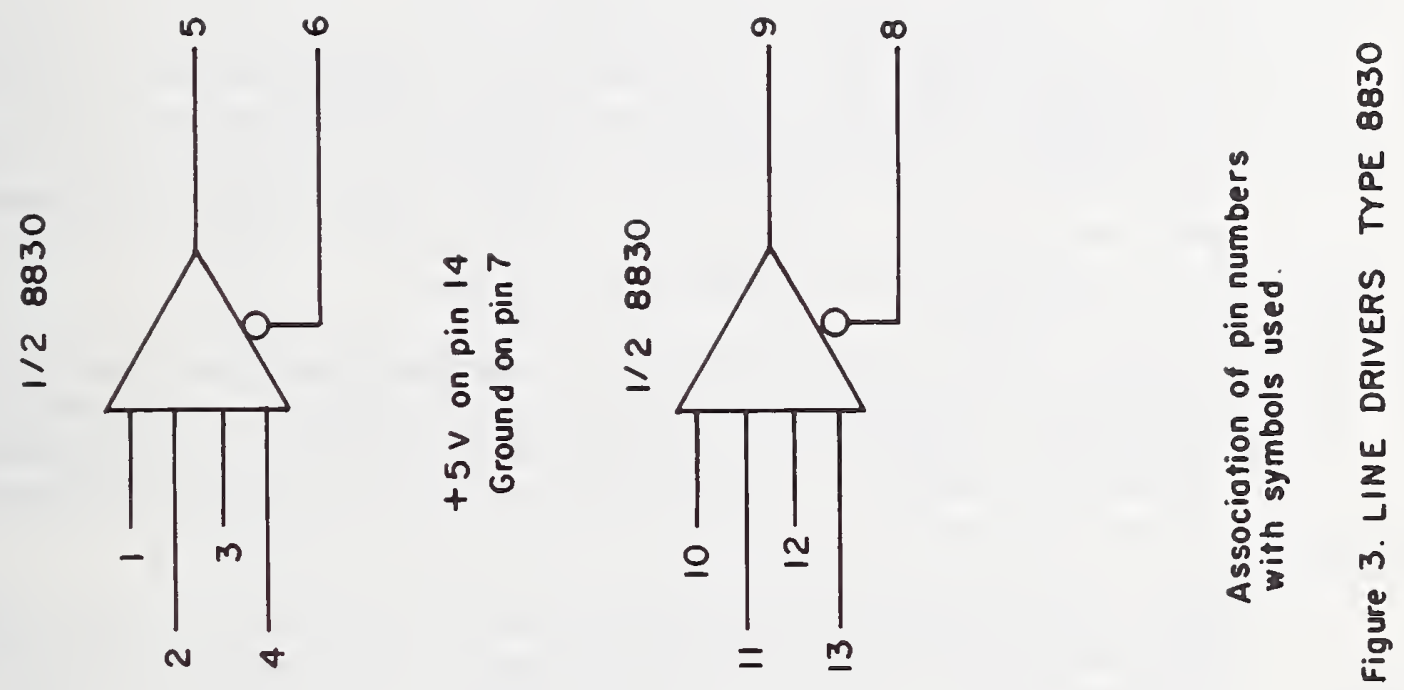
was opened. This helped capture the label more quickly, allowing the shoe to fully secure the label before the shearing action was initiated.

Another problem manifested itself frequently, resulting in the labeler performing the stamp operation before a Document was properly inserted. This problem could be relieved by making certain the document was inserted before the shoe was raised. This required extra attention from the operator and was not satisfactory on a long term basis although it was an interim measure while more permanent corrections were arranged. In some instances, the document sensing circuits were not sensitive enough to prevent logical malfunctions. Modulation of these circuits by the overhead fluorescent lighting was also a major problem causing the logic to receive incorrect signals. Correction of the document sensing problem was accomplished by a complete redesign and installation of the opto-electronic circuits.

The label drop problem was eliminated by installing a larger vacuum pump capable of increasing the system vacuum to 30 inches of water, providing at the same time a greater capacity for overcoming vacuum loss due to leakages. The size of the vacuum hose from the main distribution line to the vacuum hold-down shoe was also increased, thus reducing the vacuum loss if a label got displaced from its normal position.

In addition to this, the vacuum hold-down shoe was completely redesigned to provide a more widely distributed force pattern for label retention on the shoe. The new force distribution allows the label to be displaced somewhat without undue reduction of holding force to the point where the label drops.

The incressed vacuum and redistributed force pattern also enhanced the "capture" of the labels and the puffers were removed from the labelers. The label drop problem is also affected very strongly by the inter related positions of the label printer, vacuum hold-down shoe, and the shear mechanism. Careful adjustment of these assemblies is essential to good operation.

After a lengthy installation, adjustment, and modification period, the document labelers have been performing satisfactory service on a daily basis.

\subsection{DOCUMENT LABELER ADJUSTMENT PROCEDURES}

There are several adjustments which must be made to insure that the document labeler properly prepares and applies the labels to the documents. One is a heat adjustment, three are lamp intensities, and the remainder are mechanical adjustments. 


\subsection{Heat Adjustment}

The heat adjustment is uncomplicated. The adjusting device is a variable autotransformer capable of supplying any output voltage from zero to approximately 130 volts. A dial is provided and is calibrated from 0 to 100 . No facilities are provided for measuring the temperature of the heated vacuum hold-down shoe and no definite operating temperature has been specified. The proper heat is that which assures the label will be completely affixed to the document and remain without loosening at a later time. Experience has shown that a dial setting of approximately 80 will accomplish this.

\subsection{Lamp Intensity Adjustments}

Three document sensing lamp intensity adjustments must be made. The document sensing circuitry is shown on Drawing No. 8.3.1. The adjusting potentiometers, light amplifier circuits and test points for checking are all contained in a small box mounted on top of the shelf-mounting angle. Rotating the adjustment potentiometer clockwise increases the lamp intensity. The procedure for adjusting each lamp is identical.

Connect an oscilloscope between the amplifier output (test point) and ground (test point). Increase the lamp intensity until the amplifier output voltage is less than 0.2 volt. It is preferable to have this voltage as low as possible and still be consistent with the following test.

Insert a piece of thin paper into the light beam and observe the output voltage. The voltage should be at least 4 volts. Decreasing the lamp intensity increases this voltage.

Recheck the output voltage after removing the paper. If the output voltage does not fall to less than 0.2 volt, the lamp intensity has been decreased too much. Inability to achieve satisfactory voltage levels in both states indicates a problem with circuitry or components.

The existence of waveforms on the oscilloscope indicates that the circuitry is being modulated by the fluorescent ceiling lights. This can occur if the lamp does not have enough intensity and usually can be cured by replacement of the lamp.

It is necessary to use the oscilloscope for the above adjustments in order to determine the presence or absence of light modulation effects which would result in improper 
signals being sent to the sequencer logic.

The above adjustments are in opto-electronic circuits employed to determine when a document is properly inserted in the document labeler. When this is the case, signals are sent to the labeler sequencer logic which in turn causes the final function of the labeler to be initiated, i.e., affixing the completed label on a properly positioned document.

\subsection{Mechanical Adjustments}

The basic success or failure of the labeling process is controlled primarily by the relative positioning of three major mechanical assemblies attached to the main support plate: the printing assembly, the label shearing assembly, the label carrier assembly. The label carrier assembly should be adjusted first and the other two assemblies adjusted relative to the label carrier. The label carrying surface of the heated vacuum hold-down shoe must be in a plane perpendicular to the main support plate when the label carrier arm is in the down position. Perpendicularity of the carrier arm is controlled by the position of the actuator motor.

Place the label carrier arm in its down position by rotating the actuator motor armature manually until the actuator bell crank and the connecting rod have their axes in a straight line. Place a try square in the angle between the main plate and the label carrier arm and check for a $90^{\circ}$ angle. If adjustment is required, loosen the 4 actuator motor mounting screws and move the actuator motor up or down as necessary to obtain a $90^{\circ}$ angle. Retighten the actuator motor mounting screws taking care to see that the mechanism operates freely after tightening the screws.

To adjust the shearing mechanism, one should first lower the label carrier arm to its down (horizontal) position by rotating the actuator motor armature. Loosen the screws holding the shear support. The position of the label shear support is then adjusted in a right or left direction so the shear blade is as close to the shoe as possible without the likelihood of the blade catching on the shoe during the down stroke. At the same time this is done, the label shear support should be moved inward (toward the main support plate) or outward so the cutting edge of the shear blade is below the top of the shoe when the shear is in its downmost position. The shear support should be no further toward the main plate than necessary so that there is clearance for relative motion between the shear assembly and the label carrier assembly. Retighten the screws for the shear support plate 
and recheck adjustments. Minor adjustment may be necessary after observing the document labeler operate in its normal mode or under control of the labeler testing device.

The printer location may now be adjusted. The printer is mounted on four standoffs and is attached to these standoffs by two \#6-32 screws into each of the lower standoffs and by a captive \#6-32 thumbscrew into each of the upper standoffs. The printer must be adjusted to a position such that the label is fed out over the heated vacuum shoe to be captured by the vacuum hold-down shoe and cut off by the label shear. The label must barely clear the top surface of the shoe as it is fed out of the printer. Sliding the printer up and down on its mounting screws effects this adjustment. This adjustment is made easier if a 0.010" thick piece of shim stock is used between the printer tape guides to indicate when the top of the shoe and the top of the lower tape gulde are in the same plane.

To prevent the shear from displacing the label from the shoe, it is essential for the ends of the printer tape guides to be as close as possible to the shoe without interfering with the travel of the shearing blade. Ideally, this gap should be no greater than $0.020^{\prime \prime}$. Unfortunately, it will not usually be obtainable due to the dynamic motion of the shearing mechanisms. When the shear is energized, the yoke and blade are given a sideways motion toward the printer due to the off-center loading. This motion is permitted by the solenold mounting which must allow some looseness to prevent binding of the yoke in mounting. Extreme looseness is not desirable and must be reduced to an amount just sufficient to allow the shear to operate without binding. This looseness is controlled by the clearances between the solenoid mounting blocks. Shims can be installed between the solenoid and either mounting block as necessary to provide enough operating clearance.

There is another significant and critical adjustment to be made. The actuator cam on the actuator motor shaft must be positioned so that the shoe always stops at its proper $90^{\circ}$ angle with the main plate. The position of the cam on the actuator motor shaft determines where the shoe stops.

Connect the labeler tester to the labeler, energize the labeler and tester and position the shoe in its down position. To position the shoe first press the Reset button and then the Operate button on the Labeler Tester. The shoe will move from the up position to the down position and the printer will begin to print. At this time, turn of the labeler power switch. The shoe will remain in the down position. Observe by looking at the actuator bell crank 
and the connecting rod whether their axes are aligned and check perpendicularity of the label carrier arm with the main plate with a small try square. If not perpendicular or mechanical alignment not straight, the actuator cam must be adjusted so the shoe comes to rest at the down position as adjusted previously. Loosen the two set screws in the cam hub, rotate the cam a few degrees and retighten set screws. Turn labeler on and reposition shoe as before. Remeasure and readjust until proper adjustment has been achieved.

The above procedures will be sufficient for most adjustments on the document labeler. General knowledge of the labeler and experience with its operation will indicate when readjustment may be needed or additional adjustments required. 
TABLE 1

\section{Integrated Circuits Used}

15 volt circuits:

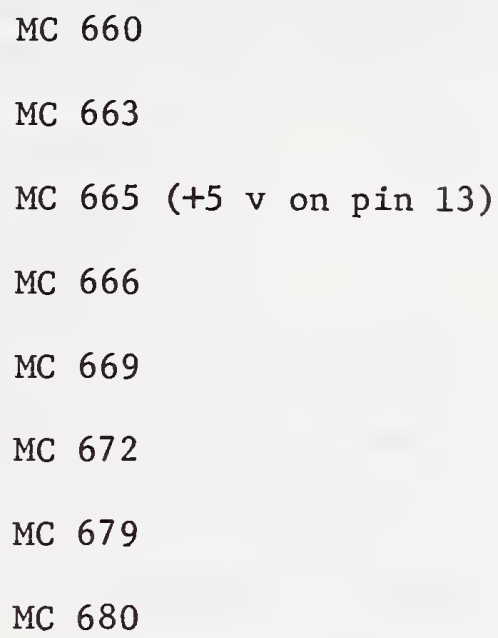


TABLE 2

Signal Location Guide

Signa1/Name ADCN

BRK

COUNTER

DATA PATH

DCEN

DCFIN

DCLMP

DCPOS

DPT

DTFF

FWD

GDE

GLST

HLTF

HLTR

LABST

LBFN

LWR

NOVC

PAP

PAPOT
Explanation Add Counter

Brake

8.2 .3

8.2 .7

8.2 .9

RPD

8.2 .12

8.2 .13

Document Lamp

Document Positioned

8.2 .4

8.2 .3

8.2 .3

Data Transfer

Flip Flop

Forward

8.2 .5

8.2 .1

Gated Document

Enable

Gated Label Start

Halt Forward

8.2 .1

8.2 .8

8.2 .8

RPD

8.2 .12

8.2 .2

8.2.11

Printer

8.2 .15

Paper Out
8.2 .7

8.2 .3

8.2 .8

Printer

8.2 .1

RPD

Visua 1

8.2 .4

RPD

$8.2 .3, .5, .11$

$8.2 .3, .5$ Printer

$8.2 .1, .2$

$8.2 .1, .3, .5$

8.2 .3

8.2 .5

8.2 .1

$8.2 .12, .13$

8.2 .2

RPD

8.2 .15

RPD 
TABLE 2 (Cont'd)

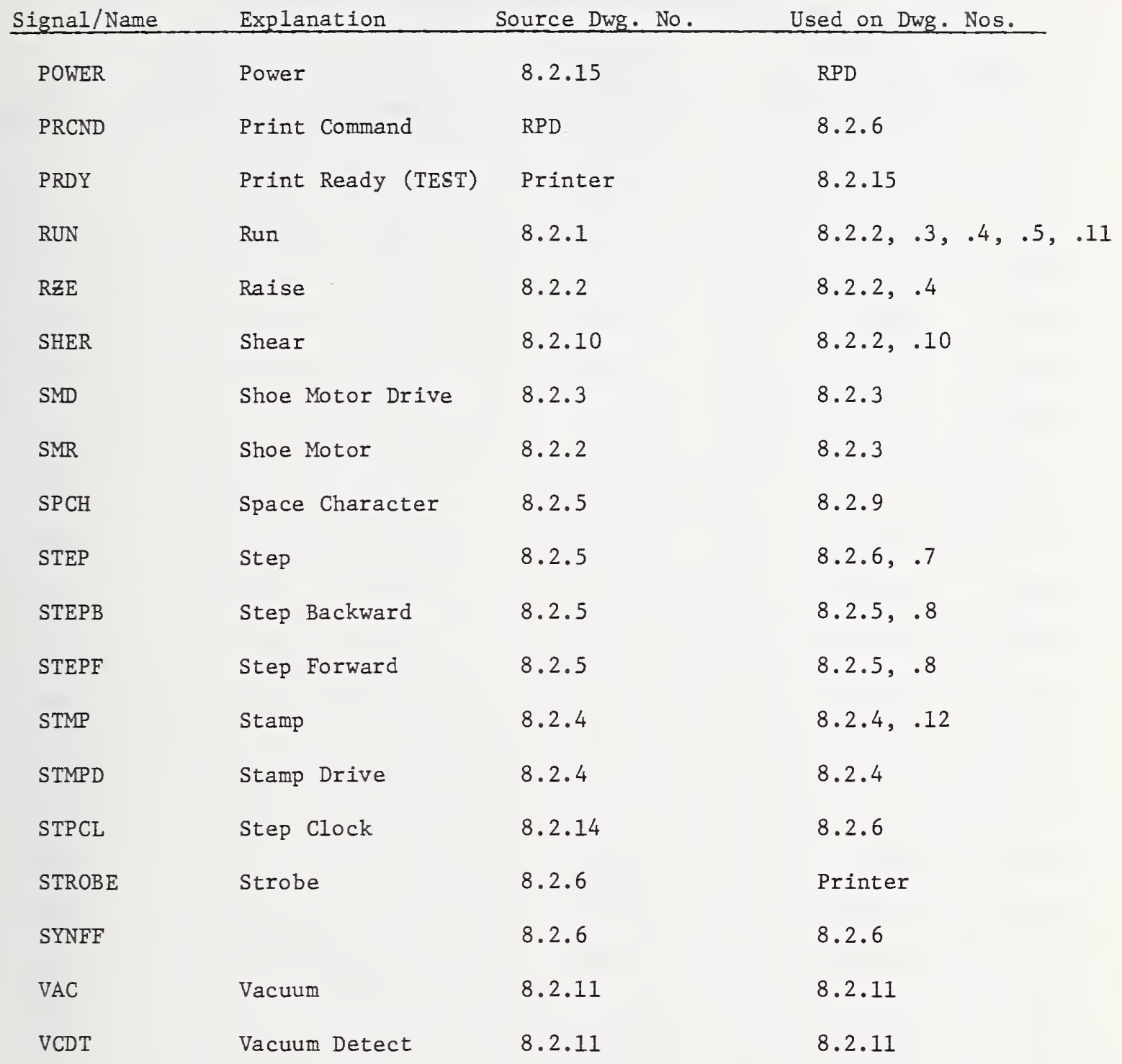


TABLE 3

Wiring from Sequencer Logic to RPD Logic

\begin{tabular}{|c|c|c|c|c|c|}
\hline Signal & $\begin{array}{l}\text { Source } \\
\text { LBLG } \\
\text { DWG. }\end{array}$ & $\begin{array}{c}\text { Pin } \\
\text { №. } \\
\end{array}$ & $\begin{array}{l}\text { Sequencer } \\
\text { Logic } \\
\text { Connector }\end{array}$ & $\begin{array}{l}\text { Labeler } \\
\text { Cable } \\
\text { Connector }\end{array}$ & $\begin{array}{c}\text { RPD } \\
\text { Cable } \\
\text { Connector } \\
\end{array}$ \\
\hline LABST & 8.2 .1 & 3.1 .3 & 1.1 .1 & A & A \\
\hline LABST- & 8.2 .1 & 3.1 .1 & 1.1 .2 & B & B \\
\hline DCEN & 8.2 .1 & 3.1 .11 & 1.1 .3 & C & C \\
\hline DCEN- & 8.2 .1 & 3.1 .13 & 1.1 .4 & D & D \\
\hline PRCMD & 8.2 .6 & 3.3 .11 & 1.1 .5 & $\mathrm{H}$ & $\mathrm{H}$ \\
\hline PRCMD- & 8.2 .6 & 3.3 .13 & 1.1 .6 & $\mathrm{~J}$ & $\mathrm{~J}$ \\
\hline Data $2^{\circ}$ & 8.2 .9 & 3.3 .3 & 1.1 .7 & K & K \\
\hline Data $2^{\circ}-$ & 8.2 .9 & 3.3 .1 & 1.1 .8 & $\mathrm{~L}$ & $\mathrm{~L}$ \\
\hline Data $2^{1}$ & 8.2 .9 & 3.6 .11 & 1.1 .9 & M & M \\
\hline Data $2^{1}-$ & 8.2 .9 & 3.6 .13 & 1.1 .10 & N & N \\
\hline Data $2^{2}$ & 8.2 .9 & 3.6 .3 & 1.1 .11 & $P$ & $P$ \\
\hline Data $2^{2}-$ & 8.2 .9 & 3.6 .1 & 1.1 .12 & $R$ & $\mathrm{R}$ \\
\hline Data $2^{3}$ & 8.2 .9 & 3.7 .3 & 1.1 .13 & $S$ & S \\
\hline Data $2^{3}-$ & 8.2 .9 & 3.7 .1 & 1.1 .14 & $\mathrm{~T}$ & $\mathrm{~T}$ \\
\hline Data $2^{4}$ & 8.2 .9 & 3.7 .11 & 1.1 .15 & U & V \\
\hline Data $2^{4}-$ & 8.2 .9 & 3.7 .13 & 1.1 .16 & V & $\mathrm{U}$ \\
\hline DPT & 8.2 .3 & 3.2 .5 & 1.1 .17 & Y & Y \\
\hline DPT- & 8.2 .3 & 3.2 .6 & 1.1 .18 & $z$ & $z$ \\
\hline DCFIN & 8.2 .12 & 3.9 .5 & 1.1 .19 & a & a \\
\hline DCFIN- & 8.2 .12 & 3.9 .6 & 1.1 .20 & $\mathrm{~b}$ & $\mathrm{~b}$ \\
\hline PAPOT & 8.2 .15 & 3.9 .9 & 1.1 .21 & c & c \\
\hline PAPOT- & 8.2 .15 & 3.9 .8 & 1.1 .22 & $\mathrm{~d}$ & $\mathrm{~d}$ \\
\hline
\end{tabular}


Table 3 Cont'd

$\begin{array}{llccccc}\text { Signal } & \begin{array}{c}\text { Source } \\ \text { LBLG } \\ \text { DWG }\end{array} & \begin{array}{c}\text { Pin } \\ \text { No. }\end{array} & \begin{array}{c}\text { Sequencer } \\ \text { Logic } \\ \text { Connector }\end{array} & \begin{array}{c}\text { Labeler } \\ \text { Cable } \\ \text { Connector }\end{array} & \begin{array}{c}\text { RPD } \\ \text { Cable } \\ \text { Connector }\end{array} & \begin{array}{c}\text { RPD } \\ \text { DWG No. }\end{array} \\ \text { NOVC } & 8.2 .11 & 3.2 .9 & 1.1 .23 & \mathrm{~h} & \mathrm{~h} & 65 \\ \text { NOVC- } & 8.2 .11 & 3.2 .8 & 1.1 .24 & \mathrm{j} & \mathrm{j} & 65 \\ \text { POWER } & 8.2 .15 & 3.2 .14 & 1.1 .25 & \mathrm{k} & \mathrm{k} & 65 \\ \text { POWER- } & 8.2 .15 & 3.5 .6 & 1.1 .26 & 1 & 1 & 65\end{array}$


TABLE 4

$$
\text { Wiring from Labeler }
$$

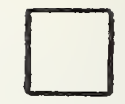

Sequencer Logic to Electro-Mechanical Devices

\begin{tabular}{|c|c|c|c|c|}
\hline Signal & DWG ${ }^{\text {So }}$ & Pin No. & $\begin{array}{c}\text { Sequencer Logic } \\
\text { Connector }\end{array}$ & $\begin{array}{r}\text { Board } \\
\text { Ter }\end{array}$ \\
\hline SHDN & 8.2 .2 & 1.8 .10 & 1.13 .1 & 7 \\
\hline SHUP & 8.2 .2 & 1.8 .12 & 1.13 .2 & 8 \\
\hline SMD & 8.2 .3 & 1.11 .8 & 1.13 .3 & 10 \\
\hline BRK & 8.2 .3 & 1.11 .3 & 1.13 .4 & 11 \\
\hline STMPD & 8.2 .4 & 1.18 .8 & 1.13 .5 & 14 \\
\hline SHERD & 8.2 .10 & 2.1 .3 & 1.13 .6 & 15 \\
\hline VCSW & 8.2 .11 & 2.1 .10 & 1.13 .7 & 16 \\
\hline VCDT & 8.2 .11 & 2.4 .3 & 1.13 .8 & -- \\
\hline DCLMP & 8.2 .13 & 2.16 .6 & 1.13 .9 & 18 \\
\hline BTDT & 8.2 .4 & 1.6 .8 & 1.13 .10 & 19 \\
\hline ENDT & 8.2 .4 & 1.6 .5 & 1.13 .11 & 20 \\
\hline
\end{tabular}


TABLE 5

Wiring from Sequencer Logic to CTS LABEL Printer

\begin{tabular}{|c|c|c|c|c|}
\hline Signal & $\begin{array}{l}\text { Source } \\
\text { DWG. }\end{array}$ & Pin No. & $\begin{array}{l}\text { Sequencer } \\
\text { Logic } \\
\text { Connector }\end{array}$ & $\begin{array}{c}\text { Printer } \\
\text { Conn. } \\
\mathrm{JI}\end{array}$ \\
\hline FRRV & 8.2 .5 & 1.18 .3 & 1.19 .1 & 12 \\
\hline STROBE & 8.2 .6 & 2.1 .8 & 1.19 .2 & 7 \\
\hline DATA $2^{\circ}$ & 8.2 .9 & 3.5 .8 & 1.19 .3 & 1 \\
\hline DATA $2^{1}$ & 8.2 .9 & 3.5 .4 & 1.19 .4 & 2 \\
\hline DATA $2^{2}$ & 8.2 .9 & 3.5 .2 & 1.19 .5 & 3 \\
\hline DATA $2^{3}$ & 8.2 .9 & 3.5 .12 & 1.19 .6 & 4 \\
\hline DATA $2^{4}$ & 8.2 .9 & 3.5 .10 & 1.19 .7 & 5 \\
\hline DATA $2^{5}$ & 8.2 .9 & $5 v$ & 1.19 .8 & 6 \\
\hline $\begin{array}{l}\text { Print Ready } \\
\text { (Test Only) }\end{array}$ & -- & -- & 1.19 .9 & 8 \\
\hline PAPER OUT & 8.2 .15 & 3.9 .13 & 1.19 .10 & 11 \\
\hline SPARE & -- & -- & 1.19 .11 & 10 \\
\hline SPARE & -- & -- & 1.19 .12 & 13 \\
\hline SPARE & -- & -- & 1.19 .13 & 14 \\
\hline $\begin{array}{l}+5 \mathrm{v} \\
\text { (Test Only) }\end{array}$ & -- & -- & 1.19 .14 & 15 \\
\hline GROUND & -- & -- & 1.19 .15 & 9 \\
\hline
\end{tabular}


The cooperation and active support of many individuals participating in formulating the system design concept, logic circuit design and fabrication of this equipment were required. In particular, we wish to thank Mr. Bernard Radack and other members of the Systems Development Division of the Internal Revenue Service for the opportunity to participate in the development of this system. The authors wish also to acknowledge contributions of other members of the Institute for Computer Sclences and Technology:

\author{
Ernest F. Ainsworth \\ Thomas L. Childs \\ Arthur A. Ernst \\ David C. Friedman \\ Thomas B. Hall \\ Ural T. Jones \\ James E. McNally \\ Louis J. Palombo \\ James N. Strohlein
}

\begin{abstract}
Permission from the Teletype Corporation to reproduce portions of certain drawings of the Model 37 RO Teletype modifications is gratefully acknowledged.
\end{abstract}

NOTE: In several instances precise identification of a product(s) has been made because the product is a part of an assembly or device and is a part whose physical dimensions or technical characteristics must, in the even of injury or failure, be duplicated exactly in a successful replacement part. In no case does such identification imply recommendation or endorsement by the National Bureau of Standards, nor does it imply that the material or equipment identified is necessarily the best available for the purpose. 
In view of the present accepted practice in this country for building technology, common US units of measurement have been used throughout this paper. In recognition of the position of the United States as a signatory to the General Conference on Weights and Measures, which gave official status to the metric SI system of units in 1960, assistance is given to the reader interested in making use of the coherent system of SI units by giving conversion factors applicable to US units used in this paper.

\section{Length}

1 in $=0.0254$ meter $($ exactly)

$1 \mathrm{ft}=0.3048$ meter $($ exactly)

\section{Force}

$1 \mathrm{lb}(1 \mathrm{bf})=4.448$ Newton $(\mathrm{N})$

$1 \mathrm{kip}=4448$ Newton

\section{Pressure}

$1 \mathrm{psi}=6895 \mathrm{~N} / \mathrm{m}^{2}$

$1 \mathrm{ksi}=6.895 \times 10^{6} \mathrm{~N} / \mathrm{m}^{2}$

Mass

$11 \mathrm{~b}=0.45359237$ kilogram $(\mathrm{kg})$ 



\begin{tabular}{|c|c|c|c|c|}
\hline $\begin{array}{l}\text { U.S. DEPT. } \\
\text { BIBLIOGRAP } \\
\text { SHE }\end{array}$ & $\begin{array}{l}\text { OF COMM. } \\
\text { PIC OATA } \\
\text { ET }\end{array}$ & $\begin{array}{l}\text { 1. PUBL.ICATION OR REPORT NO. } \\
\text { NBSIR 74-577-1 }\end{array}$ & $\begin{array}{l}\text { 2. Gov't Accession } \\
\text { No. }\end{array}$ & 3. Recipient's Accession No. \\
\hline \multirow{2}{*}{\multicolumn{4}{|c|}{$\begin{array}{l}\text { 4. TITLE AND SUBTITLE } \\
\text { REMITTANCE PROCESSING SYSTEM }\end{array}$}} & $\begin{array}{l}\text { 5. Publication Date } \\
\text { September 17, } 1974\end{array}$ \\
\hline & & & & $\begin{array}{l}\text { 6. Pesforming Organization Code } \\
650.01\end{array}$ \\
\hline \multicolumn{4}{|c|}{ 7. AUTHOR(S) Philip D. Shupe, Jr; Paul Meissner; James R. Park } & $\begin{array}{l}\text { 8. Performing Organ. Report No. } \\
\text { NBSIR } 74-577-1\end{array}$ \\
\hline \multirow{2}{*}{\multicolumn{4}{|c|}{$\begin{array}{l}\text { 9. PERFORNING ORGANIZATION NAME AND ADDRESS } \\
\text { NATIONAL BUREAU OF STANDARDS } \\
\text { DEPARTMENT OF COMMERCE } \\
\text { WASHINGTON, D.C. 20234 }\end{array}$}} & $\begin{array}{l}\text { 10. Project/Task/Work Unit No. } \\
6504567\end{array}$ \\
\hline & & & & $\begin{array}{l}\text { 11. Contract/Grant No. } \\
\text { IRS Cont. 72-487, } \\
73-558 \text {, and } 74-1013\end{array}$ \\
\hline \multirow{2}{*}{\multicolumn{4}{|c|}{ 12. Sponsoring Organization Name and Complete Address (Street, City, State, ZIP) }} & $\begin{array}{l}\text { 13. Type of Report \& Period } \\
\text { Covered } \\
\text { Interagency Report }\end{array}$ \\
\hline & & & & 14. Sponsoring Agency Code \\
\hline
\end{tabular}

15. SUPPLEMENTARY NOTES

16. ABSTRACT (A 200word or less lactual summary of most sienificant information. If document includes a significant bibliography or literefure survey, mention it here.)

The Remittance Processing System is a prototype developed for the Internal Revenue Service to test a new concept in automating the processing of taxpayer remittances. Central to the new processing concept was the integration of the Direct Data Entry System. Initial Entry Station with equipment components that would eliminate a number of Individual manual operations, permit operator interface with the central DDES computer, and provide for verification of initial entry, check endorsement, audit trail data, remittance documentation, and affixing information to taxpayer documentation accompanying the remittance. This report describes the equipment which was configured to implement these processes and to demonstrate the viability of the concept.

17. KEY WORDS (oix to twelve entries; alphabetical order; capitalize only the first letter of the first key word unless a proper name; separated by emicolons)

Audit tra11; check endorsement; check handling; data entry; document labeling; labeling; 11sting; man machine interface; MICR printing; remittance processing; remittance registe

18. AVAILABILITY [X] Unlimited

For Official Distribution. Do Not Release to NTIS

[ Order From Sup. of Doc., U.S. Government Printing Office Washington, D.C. 20402, SD Cat. No.C13

Order From National Technical Information Service (NTIS) Springfield, Virginia 22151

\begin{tabular}{|c|c|}
\hline $\begin{array}{c}\text { 19. SECURITY CLASS } \\
\text { (THIS REPURT) } \\
\text { X }\end{array}$ & 194 \\
UNCL ASSIFIED & NO. OF PAGES \\
\hline $\begin{array}{l}\text { 20. SECURITY CLASS } \\
\text { (THIS PAGE) } \\
\text { UNCLASSIFIED }\end{array}$ & 22. Price \\
\hline
\end{tabular}




Q 